\title{
THE DUALITY CASCADE
}

\author{
MATTHEW J. STRASSLER \\ Department of Physics \\ University of Washington \\ Box 351560 \\ Seattle, WA 98195 \\ E-mail: strassler@phys.washington.edu
}

\begin{abstract}
The duality cascade, and its dual description as string theory on the warped deformed conifold, brings together several sophisticated topics, some of which are not widely known. These lectures, which contain a number of previously unpublished results, and are intended for experts as well as students, seek to explain the physics of duality cascades. Seiberg duality is carefully introduced, with detailed attention to the physical implications of duality away from the far infrared. The conifold is briefly introduced and strings on the conifold (the Klebanov-Witten model) are discussed. Next, fractional branes are introduced. The duality cascade is then constructed in field theory and in its dual supergravity description. Among the newly published results: it is shown why supergravity sees the cascade as smooth; how the two holomorphic couplings (dilaton and integrated two-form in supergravity) are related to the three physical couplings in the gauge theory; that there are actually twice as many approximate fixed points in the cascade as might be naively expected. These notes are based on lectures given at TASI 2003 and at the 2003 PIMS Summer School on Strings, Gravity \& Cosmology.
\end{abstract}

\section{Contents}

1 Renormalization Group Flow and Seiberg Duality 3

1.1 Introduction: A Careless Rendering of Seiberg Duality . . . . . . . 3

1.2 Classical and Quantum Beta Functions . . . . . . . . . . . . 5

1.3 Beta Functions in Supersymmetric Theories . . . . . . . . . . . . . 7

1.4 Quartic Operators and Their Significance . . . . . . . . . . . . 12

1.5 Relevant Operators and the Seiberg-Dual Theory . . . . . . . . . . 17

1.6 Seiberg Duality, Precisely . . . . . . . . . . . . . . 21

1.7 Seiberg Duality versus Electric-Magnetic Duality . . . . . . . . . . 24

1.8 Why Seiberg Duality can be Exact, and When . . . . . . . . . 25

1.9 Exactly Marginal Operators . . . . . . . . . . . . . . . . 28 
2 The Conifold Theory $\quad 36$

2.1 The Field Theory . . . . . . . . . . . . . . . . . . . . 36

2.2 D3-branes on the Conifold . . . . . . . . . . . . . . . . . . . 42

2.3 The Maldacena Limit . . . . . . . . . . . . . . . . . . . . . . . . . 48

3 The Cascade: Preliminaries $\mathbf{5 2}$

3.1 The Base of the Cascade . . . . . . . . . . . . . . . . . . 52

3.2 The Base with an Extra D3 . . . . . . . . . . . . . 53

3.3 More Integer D-branes: $S U(N+M) \times S U(N) \ldots \ldots \ldots$

3.4 The Lowest Step of the Cascade . . . . . . . . . . . . . . . . . . 59

3.5 Another Step . . . . . . . . . . . . . . . . . 61

4 The Cascade: Descending the Grand Staircase 63

4.1 The Upper Reaches of the Cascade . . . . . . . . . . . . . . . . 63

4.2 Near the Boundary of the Valley . . . . . . . . . . . . . . . . 67

4.3 Far from the Boundary of the Valley . . . . . . . . . . . . . . . 73

4.4 SUGRA and the Flow at Large $k \ldots \ldots$. . . . . . . . . . . . . . . . . . . . . . . . 89

4.5 The End of the Cascade . . . . . . . . . . . . . . . . . . 82

4.6 Goldstone modes . . . . . . . . . . . . . . . . . . . . . . 86

4.7 Final Thoughts . . . . . . . . . . . . . . . . . 88

$\begin{array}{lr}\text { References } & 90\end{array}$ 


\section{Renormalization Group Flow and Seiberg Duality}

\subsection{Introduction: A Careless Rendering of Seiberg Duality}

What is Seiberg duality? ${ }^{1}$ Let us begin with a short summary.

Consider, first, a theory we will call "SQCD," a supersymmetric version of QCD. The model involves $\mathcal{N}=1$ supersymmetric $S U(N)$ gauge theory, with matter consisting of $N_{f}$ flavors of quarks and squarks. The left-handed quarks $\psi^{r}$ and their partner squarks $Q^{r}$, transforming in the $\mathbf{N}$ representation of $S U(N)$, are organized into $N_{f}$ chiral multiplets, also labelled $Q^{r}$, where $r=1, \ldots, N_{f}$. Note the gauge indices are not shown. The flavor indices indicate that the $Q^{r}$ transform as an $\mathbf{N}_{\mathbf{f}}$ of an $S U\left(N_{f}\right)$ flavor group, which we will call $S U\left(N_{f}\right)_{L}$, to distinguish it from the $S U\left(N_{f}\right)_{R}$ flavor group under which the left-handed antiquarks $\tilde{\psi}$ and their partner antisquarks $\tilde{Q}$ transform. These fields form $N_{f}$ chiral multiplets $\tilde{Q}_{u}$, $u=1, \ldots, N_{f}$, transforming in the $\overline{\mathbf{N}}$ of the $S U(N)$ gauge group. Note that $r$ and $u$ are indices in different groups, and cannot be contracted to make a flavor singlet; contracting them would break the flavor symmetry to the diagonal $S U\left(N_{f}\right)$. (By convention, we may take $Q^{r}$ to transform in the $\mathbf{N}_{\mathbf{f}}$ of $S U\left(N_{f}\right)_{L}$ and $\tilde{Q}_{u}$ to transform in the $\overline{\mathbf{N}}_{\mathbf{f}}$ of $S U\left(N_{f}\right)_{R}$.)

In analogy to QCD, the theory has a baryon number symmetry $U(1)_{B}$ under which $Q^{r}$ has charge $1 / N$ and $\tilde{Q}_{u}$ has the opposite charge. In addition, there is an axial $U(1)$, but this is anomalous, as in QCD. However, because of the gluinos, SQCD has a non-anomalous axial symmetry, a socalled $U(1)_{\mathcal{R}}$, under which the gluinos $\lambda$ have charge 1 , the squarks $Q$ and $\tilde{Q}$ have charge $1-\frac{N}{N_{f}}$, and the quarks $\psi$ and $\tilde{\psi}$ have charge $-\frac{N}{N_{f}}$. The action of SQCD is very simple: it consists of the kinetic terms for the fields, including the minimal couplings to the gauge fields, plus the minimal number of additional terms required by supersymmetry. In particular, the superpotential $W(Q, \tilde{Q})$ is zero.

Exercise: Verify that instantons, which have $2 N$ gluino zero modes and 1 zero mode for each $\psi$ and each $\tilde{\psi}$, are indeed invariant under the above-

\footnotetext{
${ }^{1}$ I assume that you, the reader, already know something about quantum field theory, gauge theory, supersymmetry and how to build supersymmetric gauge theories. If you don't, you'll find these lectures too advanced; I recommend you first read my TASI 2001 lectures, or Ken Intriligator's lectures in this volume, or any number of suitable texts on supersymmetry. I also assume you know some string theory and about D-branes and AdS/CFT; you may want to consult the lectures by Maldacena and by Kachru in this volume, or the review article by Aharony et al.
} 
mentioned R-symmetry. [You may wish to consult Ken Intriligator's lectures.]

Now consider a different theory, which we will call "SQCD+M" (a terminology which is not standard, but will prove useful.) This theory has gauge group $S U(\tilde{N})$, with $\tilde{N} \equiv N_{f}-N$. It also has $N_{f}$ flavors in the fundamental representation of the gauge group, labelled $q_{r}$ and $\tilde{q}^{u}$; notice the location of the indices is different, indicating that $q_{r}$ transforms as an $\overline{\mathbf{N}}_{\mathbf{f}}$ of $S U\left(N_{f}\right)_{L}$ and $\tilde{q}^{u}$ as an $\mathbf{N}_{\mathbf{f}}$ of $S U\left(N_{f}\right)_{R}$. But the theory is not quite SQCD again, because it also has another set of gauge-singlet chiral superfields, labelled $M_{u}^{r}$, and coupling to the matter fields by the superpotential

$$
W=y M_{u}^{r} q_{r} \tilde{q}^{u}
$$

where $y$ is a coupling constant we will discuss later. Note $M_{u}^{r}$ transforms as $\left(\mathbf{N}_{\mathbf{f}}, \overline{\mathbf{N}}_{\mathbf{f}}\right)$ of $S U\left(N_{f}\right)_{L} \times S U\left(N_{f}\right)_{R}$. The baryon number of the $q$ fields is $1 / \tilde{N}$ (the field $M$ is uncharged). Finally, there is again an anomaly-free $\mathrm{R}$ symmetry under which both $q$ and $\tilde{q}$ transform with charge $1-\frac{\tilde{N}}{N_{f}}$. The field $M$ transforms with R-charge $2 \frac{\tilde{N}}{N_{f}}$, which ensures that the superpotential $W$ has R-charge 2 , as required by supersymmetry.

In 1994, Seiberg wrote an extraordinary paper [1] arguing that SQCD with $N$ colors and $N_{f}$ flavors is dual to SQCD + M with $\tilde{N}=N_{f}-N$ colors and $N_{f}$ flavors. What does this mean?

Seiberg explained that these two theories, which are manifestly different when they are both weakly coupled, nonetheless have the same physics at low momentum (in the "far infrared"), where at least one, if not both, are strongly coupled. In particular, the Green's functions (and S-matrices, if they exist) of the two theories become identical in the limit that the external momenta are all taken to zero, as long as we match the gauge-invariant operators of one theory to those of the other. For instance, $Q^{r} \tilde{Q}_{u}$ is matched to $M_{u}^{r}$; the Green functions of $Q^{r} \tilde{Q}_{u}$ in the SQCD theory approach the Green functions of $M_{u}^{r}$ in the SQCD+M theory at low momenta.

But this is just the beginning. There are many additional consequences of duality that Seiberg did not explicitly discuss in his original paper. It is these implications - which Seiberg himself understood fully, but which are widely misunderstood - which we will seek to elucidate in the first section of these lectures.

After we understand Seiberg duality, we'll then discuss continuous spaces of conformal field theories, then combine this with AdS/CFT (discussed in Maldacena's lectures), and finally more general gauge the- 
ory/string theory dualities. Finally, the whole set of tools will be brought to bear in the context of strings propagating on the so-called "warped deformed conifold," a space rather more beautiful than its name implies, and the duality of this theory with a quantum field theory exhibiting a "duality cascade," a sequence of Seiberg dualities.

\subsection{Classical and Quantum Beta Functions}

If you want to understand what it means for two theories to become identical in the infrared, the first thing you must do is ensure your understanding of renormalization is complete. What precisely is a beta function? What precisely do terms such as "relevant," "irrelevant" and "marginal" mean - irrelevant to what? What is a "dangerous irrelevant" operator, and what makes it dangerous? Why is the "renormalization group" (RG) not a group? A substantial discussion of this subject is presented in my TASI 2001 lectures [2] and I refer you to that document for details. Still, there are a few things we should review for use in these lectures.

Exercise: Give an example of a relevant operator in classical supersymmetric field theory, and define its beta function in a way which shows most clearly that it is relevant. What is the sign of its beta function?

Let's consider a massive free complex scalar field.

$$
S=\int d^{d} x\left[\partial_{\mu} \phi^{\dagger} \partial^{\mu} \phi-m^{2} \phi^{\dagger} \phi\right] .
$$

What is the renormalization group flow associated with this theory? It can't be completely trivial even though the theory is free and therefore soluble. The number of degrees of freedom is two (one complex scalar equals two real scalars) in the ultraviolet and zero in the infrared, so obviously the theory is scale dependent.

Exercise: Verify this statement by computing the propagator in position space; show it is scale invariant at extremely small and extremely large distances, but is not scale-invariant at distances of order $m^{-1}$.

Clearly the mass term is not of any importance for ultraviolet physics, but is enormously important for infrared physics. What can we do to make this intuitively obvious fact precise? The correct approach is to define a 
dimensionless coupling $\nu^{2} \equiv m^{2} / \mu^{2}$ where $\mu$ is the renormalization-group scale - the scale at which we observe the theory. Then we can think of this theory as transitioning, as in Fig. 1, between two even simpler theories: the scale-invariant theory at $\mu \rightarrow \infty$, where the mass of $\phi$ is negligible and $\nu \rightarrow 0$, and the empty though scale-invariant $\nu \rightarrow \infty$ theory in the infrared, where the scalar does not propagate.

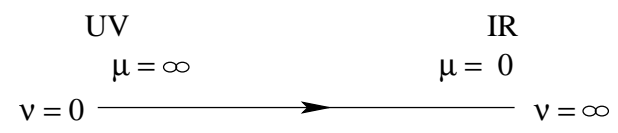

Figure 1. The effect of a mass term grows in the infrared.

In this way of thinking, scale transformations move the theory from the $\nu=0$ conformal fixed point to the empty theory at $\nu=\infty$. The arrows indicate the change in the theory as one considers it at larger and larger length scales $\mu^{-1}$.

A mass term is known as a "relevant" operator, where the relevance in question is at long distances (low energies.) Although the mass term has no effect in the ultraviolet - at short distance - it dominates the infrared (in this case by removing degrees of freedom.) We can see this from the fact that the dimensionless coupling $\nu$ grows as we scale from the ultraviolet toward the infrared. In fact we can define a beta function for $\nu=m / \mu$ as follows:

$$
\beta_{\nu} \equiv \mu \frac{\partial \nu}{\partial \mu}=-\nu
$$

That $\nu$ grows in the infrared is indicated by the negative beta function. More specifically, the fact that the coefficient is -1 indicates that $\nu$ scales like $1 / \mu$. This tells us that the mass $m$ has dimension 1 .

What about a classically irrelevant operator? A good example would be given by adding an $h \phi^{6}$ interaction to the above Lagragian. In four dimensions, the field $\phi$ has dimension 1 , so for $\int d^{4} x h \phi^{6}$ to be dimensionless, $h$ must have dimension -2 . This operator is classically irrelevant; any amplitude where the external momenta are of order $\mu$ must, by dimensional analysis, be a function of $h \mu^{2}$. But this tells us the natural coupling constant here is the dimensionless quantity $\eta=h \mu^{2}$; and

$$
\beta_{\eta} \equiv \mu \frac{\partial \eta}{\partial \mu}=+2 \eta \text {. }
$$


Here, we have a positive classical beta function, telling us that $\eta$ scales as $\mu^{2}$, and therefore the operator has negligible effect on amplitudes for which all external momenta are small.

What about operators with classically dimensionless coefficients, such as $\lambda \phi^{4}$ ? The coupling $\lambda$ is dimensionless, so its classical beta function is zero. Such a coupling is classically just as important in the infrared as in the ultraviolet, and it is called "marginal." Another example of a marginal coupling is a gauge coupling in four dimensions. ${ }^{2}$ We can summarize these various cases by noting that in all of these classical examples, the beta function for the dimensionless quantity corresponding to a coupling $y$ is simply proportional to its dimension (times a minus sign.)

Of course, these classical beta functions will get quantum corrections, which in perturbation theory will be given as a series expansion in $\eta, \lambda$ and $\nu$. As long as these couplings are small, the beta functions will not change much; the relevant operators will still be relevant, and the irrelevant operators will still be irrelevant. But marginal operators will be different! The quantum corrections can make them irrelevant (as in QED) or relevant (as in QCD) or in very rare cases leave them marginal (as in $\mathcal{N}=4$ YangMills theory - more on this below.) And when coupling constants are big, all bets are off: classically irrelevant operators can become relevant, and vice versa. Such big effects are not visible in perturbation theory, but we, armed with nonperturbative methods, will see many examples in what follows.

\subsection{Beta Functions in Supersymmetric Theories}

What happens in $\mathcal{N}=1$ supersymmetric theories? The story begins with some confusion, because there are two different types of couplings here. There are holomorphic couplings, and there are physical couplings. Holomorphic couplings, which appear in the Wilsonian effective action for these theories in an appropriate supersymmetric scheme, include the couplings which appear explicitly in the superpotential, and the gauge coupling which multiplies $\int d^{2} \theta W_{\alpha} W^{\alpha}$ in the action. There are nonrenormalization theorems which say (1) couplings in the superpotential are not renormalized at any order in perturbation theory; (2) the gauge coupling is not renormalized at any order in perturbation theory beyond one loop; (3) additional

${ }^{2}$ Note that the number of space-time dimensions affects the story! In three dimensions, the $\phi^{4}$ operator is classically relevant, as is the gauge coupling, while the operator $\phi^{6}$ is marginal. 
renormalizations are holomorphic functions and are therefore not functions of $\mu$, and so do not affect the beta functions as defined above. Naively, this seems to say that all quantum corrections to beta functions for superpotential couplings must vanish, and that

$$
\beta_{1 / g^{2}}=\frac{b_{0}}{8 \pi^{2}}
$$

exactly. (Here $b_{0}$ is the one-loop beta function coefficient; $b_{0}=3 N-N_{f}$ for supersymmetric $S U(N)$ QCD with $N_{f}$ flavors.)

But of course this is wrong, and the reason is that there is a part of the theory - the Kähler potential — which both contributes to physical Green functions and is renormalized. In particular, there is nontrivial renormalization of the coefficients of all kinetic terms (also known as wave-function renormalization); the kinetic terms take the form $\int d^{4} \theta Z_{Q} Q^{\dagger} Q$, where the wave-function coefficient $Z_{Q}$ of the chiral superfield $Q$ is a function of the coupling constants. Physical couplings are given by canonically normalizing the fields, $Q \rightarrow \sqrt{Z_{Q}} Q$, This properly normalizes the pole of a propagator of $Q$, and ensures that the couplings appearing in the Lagrangian are related to the matrix elements for scattering of the $Q$ particles. These couplings are not holomorphic, since the $Z_{Q}$ are real functions of the couplings.

Without going into detail (again the reader is referred to my TASI 2001 lectures), the fact that $Z_{Q}$ renormalizes physical Green functions, and thereby enters actual measurements, implies that there is a relation between beta functions of physical couplings involving $Q$ and the anomalous dimension $\gamma_{Q}$ of $Q$. The latter,

$$
\gamma_{Q}=-\frac{\partial \ln Z_{Q}}{\partial \ln \mu},
$$

is a function of all of the coupling constants in the theory, evaluated at a scale $\mu$ of order the momentum scale of the physical process in question. (Note $\operatorname{dim} Q=1+\frac{1}{2} \gamma_{Q}$, with a $\frac{1}{2}$ because $Z$ multiplies a bilinear in $Q$.) In particular, if a coupling $W=h \phi_{1} \phi_{2} \phi_{3}$ appears in the superpotential, (note in four dimensions this implies $h$ is classically dimensionless, since $\operatorname{dim} W=3$ and $\operatorname{dim} \phi=1$ ), then its beta function is

$$
\beta_{h}=\frac{1}{2} h\left[\gamma_{1}(h)+\gamma_{2}(h)+\gamma_{3}(h)\right] .
$$

Note that (as in the classical cases) the full quantum beta function for $h$ is proportional to its dimension (times a minus sign):

$$
\operatorname{dim} h=\operatorname{dim} W-\operatorname{dim} \phi_{1}-\operatorname{dim} \phi_{2}-\operatorname{dim} \phi_{3}=-\frac{1}{2}\left(\gamma_{1}+\gamma_{2}+\gamma_{3}\right) .
$$


This relation between quantum beta function and quantum dimension uses supersymmetry extensively. In $\mathcal{N}=1$ theories, no singularities arise when defining composite operators built as a product of chiral fields. Therefore, since $\operatorname{dim} \phi=1+\frac{1}{2} \gamma$, $\operatorname{dim}\left(\phi_{1} \phi_{2} \phi_{3}\right)=3+\frac{1}{2}\left(\gamma_{1}+\gamma_{2}+\gamma_{3}\right)$, and $\operatorname{dim} h=3-\operatorname{dim} \phi_{1}-\operatorname{dim} \phi_{2}-\operatorname{dim} \phi_{3}=-\frac{1}{2}\left(\gamma_{1}+\gamma_{2}+\gamma_{3}\right)$. And the classical relation $\beta_{h}=-h \operatorname{dim} h$, which relates to the scaling properties of the coupling, would be violated by additive renormalizations in generic theories; in this case $\beta_{h}$ could be nonzero even if $h$ were zero. Supersymmetry helps to preserve this unusually simple relation between dimensions and beta functions through its nonrenormalization theorems.

As for gauge couplings, the physical beta function is given in the NSVZ form discussed in Intriligator's lectures (and see my TASI 2001 lectures [2] for a partial derivation $)^{3}$

$$
\beta_{\frac{8 \pi^{2}}{g^{2}}}=\frac{b_{0}+\frac{1}{2} \sum_{r} \gamma_{r}+\frac{1}{2} \sum_{u} \gamma_{u}}{1-g^{2} N / 8 \pi^{2}}=-\frac{16 \pi^{2}}{g^{3}} \beta_{g}
$$

where $\gamma_{r}$ is the anomalous dimension of $Q^{r}$ and $\gamma_{u}$ that of $\tilde{Q}_{u}$. In supersymmetric QCD, where in the absence of a superpotential all charged fields are related by symmetry, and therefore have the same anomalous dimension $\gamma_{0}$, we may write

$$
\beta_{\frac{8 \pi^{2}}{g^{2}}}=\frac{3 N-N_{f}\left[1-\gamma_{0}\right]}{1-g^{2} N / 8 \pi^{2}} .
$$

The reason to write the beta function for $8 \pi^{2} / g^{2}$ instead of $g$ is merely that the former appears naturally in many nonperturbative contexts; it is also written as $\operatorname{Im}[2 \pi \tau]$, where $\tau$ is the often-defined

$$
\tau \equiv \frac{1}{2 \pi}\left[\theta+i \frac{8 \pi^{2}}{g^{2}}\right]
$$

Here $\theta$ is the usual theta angle for the gauge theory. Either form will do, as long as one keeps track of the implications. Another useful form is

$$
\beta_{g}=-\frac{g^{3}}{16 \pi^{2}} \frac{3 N-N_{f}\left[1-\gamma_{0}\right]}{1-g^{2} N / 8 \pi^{2}}
$$

which makes it clear that the beta function for $g$ starts at order $g^{3}$ even if $\gamma_{0} \neq 0$. Defining the 't Hooft coupling $\lambda \equiv g^{2} N$ (sometimes normalized

${ }^{3}$ Be careful of signs! In an asymptotically free theory like Yang-Mills theory, $\beta_{g}<0$ but $\beta_{8 \pi^{2} / g^{2}}>0$. 
with an additional $1 / 4 \pi)$, we have

$$
\beta_{\lambda}=-\frac{\lambda^{2}}{8 \pi^{2}} \frac{3-\frac{N_{f}}{N}\left[1-\gamma_{0}\right]}{1-\lambda / 8 \pi^{2}} .
$$

From this it is more evident that perturbation theory is really an expansion in $\lambda$, not $g^{2}$; as 't Hooft pointed out, perturbation theory breaks down when $\lambda \sim 1$, even if $g^{2} \ll 1$.

Let us now consider the first gauge theory in Seiberg's dual pair: $S U(N)$ SQCD, with $N_{f}$ flavors $Q^{r}, \tilde{Q}_{u}$ and $W=0$. The only coupling is the gauge coupling, and the beta function is Eq. (1.9). Note that this expression applies assuming that all expectation values of scalar fields are zero, so that all fields have zero mass. If there are any nonzero expectation values (i.e., if we are not at the origin of "moduli space," the space of supersymmetric vacua) or there are any explicit mass terms, then the above formula will be drastically modified below the mass scale of the massive particles.

When $g$ is small, $\gamma_{0}$, which is zero for $g=0$, must be small. (It turns out to be negative.) Therefore, if $N_{f}<3 N$, then for $g \ll 1$ we have $\beta_{g}<0$, and thus the coupling $g(\mu)$ will grow as $\mu$ decreases. But when $g$ becomes large (more precisely, when $\lambda \gg 1$ ) we simply don't know what happens from perturbation theory alone. The anomalous dimension $\gamma_{0}$ is an unknown function of $g$, and we simply don't know what it does.

An exception occurs when $N_{f}$ is very close to $3 N$. When $N, N_{f} \gg 1$ and $b_{0}=3 N-N_{f} \ll N$, it can be shown, working to two-loop order, that a conformal fixed point exists at $g \sim 1 / N$. The way this happens is this: the function $\gamma_{0}$ behaves as $-c g^{2} N+\operatorname{order}\left(g^{2} N\right)^{2}$, where the number $c$ is positive and of order one. From Eq. (1.9), the beta function for the gauge coupling looks like

$$
\beta_{g}=-\frac{g^{3}}{16 \pi^{2}} \frac{b_{0}+N_{f}\left[-c g^{2} N+\operatorname{order}\left(g^{2} N\right)^{2}\right]}{1-g^{2} N / 8 \pi^{2}}
$$

which then means the renormalization group (RG) flow looks like figure Fig. 2, with a zero at

$$
g=g_{*} \approx \sqrt{\frac{b_{0}}{3 c}} \frac{1}{N} .
$$

This means the 't Hooft coupling is very small: $\lambda_{*}=g_{*}^{2} N \sim 1 / N$. Since perturbation theory is an expansion in $\lambda$, all three-loop and higher corrections are suppressed by higher powers of $1 / N$. Therefore, this two-loop fixed point (sometimes called a Banks-Zaks fixed point) survives to all orders. 


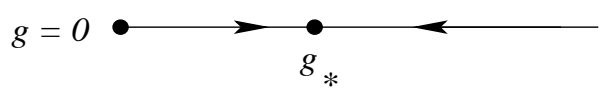

Figure 2. For $N_{f}$ in the conformal window, the gauge coupling has a stable infrared fixed point at $g=g_{*}$.

If the coupling starts at small values in the ultraviolet, it grows to $g=g_{*}$, and then stops. If it starts at larger values, it shrinks to $g_{*}$, and stops. The value $g_{*}$ is called a "fixed point" for obvious reasons; if the coupling reaches this value, it never leaves. It is called an "infrared fixed point" because it reaches this value at small $\mu$ (large distance.) And it is called stable because no matter how one perturbs $g$ away from $g_{*}$, then (if the perturbation is not too large) $g$ will flow back to $g_{*}$. (By contrast, the point $g=0$ represents an unstable ultraviolet fixed point in the context of this RG flow.)

One of Seiberg's key suggestions (still unproven!) was that this sort of fixed point exists even for $3 N-N_{f} \sim N$, in particular, that it exists for any $N_{f}$ in the range $\frac{3}{2} N<N_{f}<3 N$. In this "conformal window," the RG flow always takes the form in Fig. 2, except that $g_{*}$ is at larger values. In particular, $g_{*}$ grows as $N_{f}$ shrinks.

We will have more to say later about what happens for $N_{f} \leq \frac{3}{2} N$, but for now, let us study these fixed points further. Using some important theorems, there are a number of extremely valuable observations which one can make about these fixed points, even before considering Seiberg duality.

Here is one crucial theorem. Near any conformal fixed point (including a free field theory) all spin-zero gauge-invariant operators $\mathcal{O}$ must have dimension greater than or equal to 1 (or more generally, $(d-2) / 2$ ). If its dimension is 1 (or more generally, $(d-2) / 2$ ), then $\partial^{2} \mathcal{O}=0$ (i.e., the operator satisfies the massless Klein-Gordon equation.) This is true without any appeal to supersymmetry!

From this, it follows quickly that if all gauge couplings are zero, but $\phi$ is not free, then $\gamma_{\phi}>0$ for any field $\phi$. This is because if all gauge couplings vanish, then $\phi$ is gauge invariant, and its dimension $\operatorname{dim} \phi=1+\frac{1}{2} \gamma_{\phi}$ must be greater than one.

Here is another theorem. At an $\mathcal{N}=1$ supersymmetric conformal fixed point, there is a close relation between the dimensions of many chiral operators and the R-charges that they carry. The current of the R-symmetry and the energy-momentum tensor (of which the scale-changing operator, 
the "dilation" or "dilatation operator," is a moment) are part of a single supermultiplet of currents. At a conformal fixed point, the dilation current and the $\mathrm{R}$ current are both conserved quantities, ${ }^{4}$ and the superconformal algebra can then be used to show that the dimension of a chiral operator is simply $3 / 2$ times its $\mathrm{R}$ charge:

$$
\operatorname{dim} \mathcal{O}=\frac{3}{2} R_{\mathcal{O}}
$$

which implies that $\int d^{2} \theta \mathcal{O}$ is a relevant (irrelevant) operator at a conformal fixed point when the R-charge of $\mathcal{O}$ is less (greater) than 2.

Let's now prove [1] that in SQCD for $N_{f} \leq \frac{3}{2} N$ there Seiberg fixed points - specifically, fixed points at which no fields are free, and which are located at the origin of moduli space, where no fields have expectation values - cannot occur. In SQCD a fixed point requires

$$
\beta_{\frac{8 \pi^{2}}{g^{2}}} \propto b_{0}+N_{f} \gamma_{0}=0 \Rightarrow \gamma_{0}=1-\frac{3 N}{N_{f}} \text {. }
$$

Using the earlier formula that $\operatorname{dim} Q=1+\frac{1}{2} \gamma_{0}=\frac{3}{2} R_{Q}$, we see that we recover Seiberg's R-charge assignment,

$$
\operatorname{dim} Q=\frac{3}{2}-\frac{3 N}{2 N_{f}}, R_{Q}=1-\frac{N}{N_{f}},
$$

which he obtained using the fact that this particular R-symmetry is the unique non-anomalous chiral symmetry of the theory that commutes with all other symmetries. However, if $\gamma_{0} \leq-1$, then the gauge-invariant operator $Q^{r} \tilde{Q}_{u}$ would have dimension $2\left(1+\frac{1}{2} \gamma_{0}\right) \leq 1$. This is not allowed at a nontrivial fixed point. Thus, to have such a fixed point (at least one in the simple class we have been discussing) it must be that

$$
\gamma_{0}>-1 \Rightarrow b_{0}<N_{f} \Rightarrow N_{f}>\frac{3}{2} N
$$

Equivalently, $R_{Q}>1 / 3$.

\subsection{Quartic Operators and Their Significance}

To illustrate the concepts of relevance and irrelevance at the quantum level, let us turn to a very important operator. Consider SQCD - for reasons

${ }^{4}$ Caution: the conserved R-current at a particular fixed point may not be a symmetry in the ultraviolet. This happens in SQCD for $N_{f} \leq \frac{3}{2} N$, where the dual theory is free in the infrared and develops new symmetries, including a new R-charge, in the infrared limit. 
that will become abundantly clear, let's call this the "A" theory for the following discussion — and consider adding to it the superpotential

$$
W=h\left(Q^{r} \tilde{Q}_{u}\right)\left(Q^{u} \tilde{Q}_{r}\right)
$$

with gauge indices contracted inside the parentheses. (In this and all subsequent expressions, summation over repeated and contracted indices is implicit; indices are raised and lowered with a Kronecker delta function.) The coupling $h$ has mass dimension -1 , and thus the operator above is classically irrelevant.

Crucially, this superpotential, which explicitly breaks part of the global flavor symmetry (since $r$ and $u$ indices are contracted), still preserves a diagonal $S U\left(N_{f}\right)$ symmetry and charge conjugation. This is enough symmetry to ensure that all of the fields still share the same anomalous dimension $\gamma_{0}(g, h)$, as was true also for $h=0$. As always we should study the dimensionless coupling constant $\eta \equiv h \mu$ and ask how it scales. Classically it scales like $\mu$ (and thus has $\beta_{\eta}=\eta>0$ ) but quantum mechanically it is a different story. ${ }^{5}$ In the quantum theory, the coupling $\eta$ will have a beta function

$$
\beta_{\eta}=\eta\left[1+\frac{1}{2}\left(4 \gamma_{0}\right)\right]=\eta\left(1+2 \gamma_{0}\right),
$$

where the 1 is the classical scaling and the 4 represents the contributions of the four chiral superfields in the operator. The formula for the gauge coupling is unchanged

$$
\beta_{g} \propto-\beta_{\frac{8 \pi^{2}}{g^{2}}} \propto-\left[3 N-N_{f}+N_{f} \gamma_{0}\right] .
$$

Now, let us recall that $\gamma_{0}$ is a function of $g$ and $\eta$ with the following properties. (1) If $g=0$ and $\eta \neq 0$, then $\gamma_{0}>0$. (2) If $\eta=0$ and $0 \neq g \ll 1$, then $\gamma_{0}<0$. (3) For $3 N>N_{f}>\frac{3}{2} N$, there is at least one nontrivial fixed point at $g=g_{*}, \eta=0$ with $\gamma_{0}=1-\frac{3 N}{N_{f}}$. We can use these facts

\footnotetext{
${ }^{5}$ Wait a minute. This theory, whose potential contains (scalar) ${ }^{6}$ terms, is nonrenormalizable. Can we even discuss it quantum mechanically? Well, nonrenormalizable simply means that the operator in the superpotential is irrelevant, so in the ultraviolet regime the effective coupling is blowing up and perturbative diagrams in the theory don't make sense. But we're interested in the infrared anyway. We'll deal with the ultraviolet later; for now we will think of $1 / h$ as setting an ultraviolet cutoff on the theory. Note that we do essentially the same thing with QED in four dimensions, which is perturbatively renormalizable but nonperturbatively nonrenormalizable, since we cannot take the cutoff on the theory to infinity without the gauge coupling diverging in the ultraviolet. Perturbation theory may not converge, but we are asking perfectly valid nonperturbative infrared questions which do not depend on the details of the ultraviolet cutoff.
} 
to determine the qualitative forms (in particular, the signs and zeroes) of the beta functions. For instance, using Eq. (1.14), which changes sign at $\gamma_{0}=-\frac{1}{2}$, we see that at the nontrivial fixed point on the $\eta=0$ axis, $\eta$ is irrelevant (as it is classically) if $N_{f}>2 N$, marginal if $N_{f}=2 N$, and relevant if $N_{f}<2 N$.

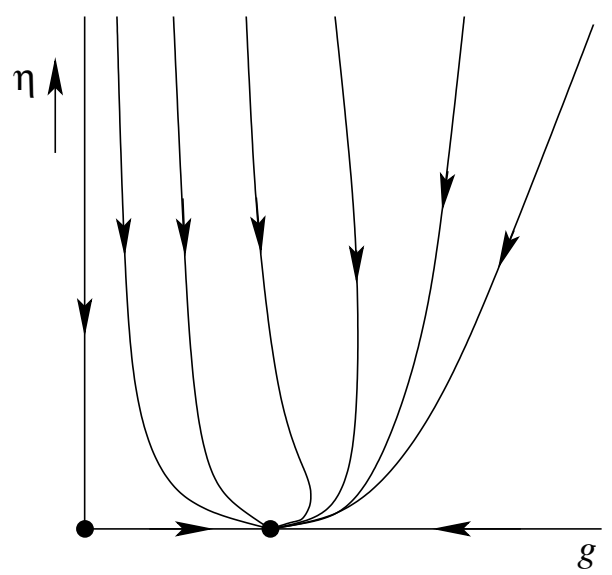

Figure 3. For $N_{f}>2 N$ the coupling $\eta$ is irrelevant both at $g=0$ and at $g=g_{*}$.

From these observations we can guess the qualitative features of the renormalization group flow. For $N>2 N_{f}$, the qualitative picture is given in Fig. 3. Even if $\eta \neq 0$, we still end up at the Seiberg fixed point. For $N<2 N_{f}$, however, there is a very different picture, as in Fig. 4 . Notice that if we start at weak gauge coupling initially, $\eta$ is irrelevant and flows toward zero as we would expect classically; but as we flow toward the infrared, the gauge coupling grows, $\gamma_{0}$ becomes more negative, and eventually the coupling $\eta$ turns around and becomes relevant. Although at first it seems as though it will be negligible in the infrared, it in fact dominates. This is called a "dangerous irrelevant" operator, since although it is initially irrelevant it is dangerous to forget about it! In the infrared it becomes large, and we must be more precise about what happens when it gets there... on this, more below.

What about $N_{f}=2 N$ ? In this case, since the operator is marginal at leading order in $\eta$ (in the same way that the operator $\phi^{4}$ is marginal in a theory of a scalar field,) we need to compute more carefully to find out 


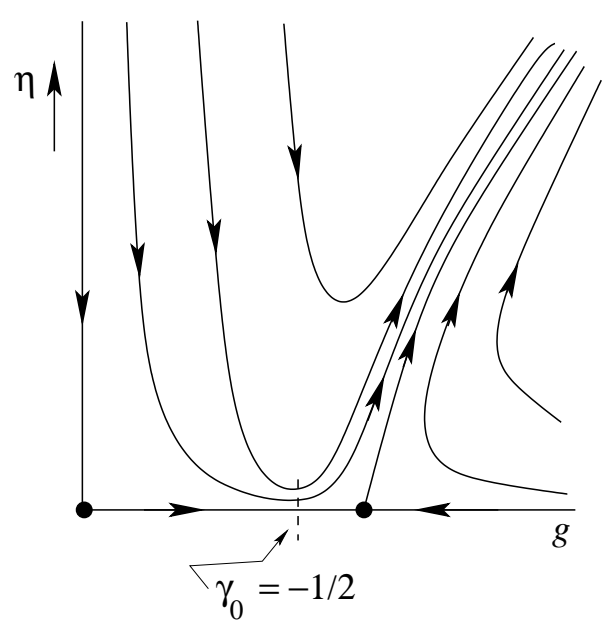

Figure 4. For $N_{f}<2 N$ the coupling $\eta$ is relevant at $g_{*}$; its initial decrease is reversed once $g$ is sufficiently large.

whether the operator is marginally relevant or marginally irrelevant. We'll do this later. For now, let's conclude our discussion of this operator by analyzing its description in the dual theory.

In the dual $S U(\tilde{N})$ SQCD + M theory, which we will now refer to as the "B" theory, the operator $\left(Q^{r} \tilde{Q}_{u}\right)\left(Q^{u} \tilde{Q}_{r}\right)$ is mapped into the operator $M_{u}^{r} M_{r}^{u}$; thus the superpotential is

$$
W=y M_{u}^{r} q_{r} \tilde{q}^{u}+\hat{h} M_{u}^{r} M_{r}^{u} .
$$

I have written $\hat{h}$ instead of $h$. Although $Q \tilde{Q}$ is mapped to $M$, the former is classically of dimension 2 and the latter is classically of dimension 1 in the ultraviolet (which is OK, since theory A and theory B are only the same in the infrared.) In other words, $h$ is proportional to $\hat{h}$ - if one vanishes, so does the other - but they are not equal. Only $\eta=h \mu$ and $\hat{\eta}=\hat{h} / \mu$ could possibly be equal, and even then, not until we approach the Seiberg fixed point.

Clearly $\hat{h}$ has units of mass, because it multiplies a mass term for $M$. And so we would expect it must be a relevant perturbation, as it is classically. Classically, we could choose to look at processes at scales below the mass of $M$. We may integrate out $M$ by using its equation of motion

$$
\bar{D}^{2}\left(M_{r}^{u}\right)^{\dagger}=y q_{r} \tilde{q}^{u}+2 \hat{h} M_{r}^{u} .
$$


The left-hand side goes to zero in the infrared, giving

$$
M_{r}^{u}=-\frac{y}{2 \hat{h}} q_{r} \tilde{q}^{u} .
$$

Substituting this into the superpotential gives the low-energy superpotential

$$
W_{L}=-\frac{y^{2}}{4 \hat{h}} q_{r} \tilde{q}^{u} q_{u} \tilde{q}_{r} \equiv \tilde{h} q_{r} \tilde{q}^{u} q_{u} \tilde{q}_{r}
$$

which, remarkably, is of the same form as the superpotential in the original A theory! except that $\tilde{h}=-y^{2} / 4 \hat{h} \propto 1 / h$.

Of course this was a classical analysis, in which we assumed $\hat{h}$ was relevant, as it is classically. But this is surely wrong. For $N_{f}>2 N$, it cannot both be that the $Q \tilde{Q} Q \tilde{Q}$ operator in the A theory is irrelevant (so that $\eta=h \mu$ flows to zero in the infrared) and that the $M M$ operator in the $\mathrm{B}$ theory is relevant (so that $\hat{\eta}=\hat{h} / \mu$ flows to infinity in the infrared, causing $\tilde{\eta}=\tilde{h} \mu$ to flow to zero). If both $\eta$ and $\tilde{\eta}$ flowed to zero, then we would learn that $S U(N)$ SQCD is dual to $S U(\tilde{N})$ SQCD with no superpotential and no neutral scalars $M$. This is simply not true. Clearly duality must somehow avoid this absurd conclusion.

Of course it does avoid it, and it does so because anomalous dimensions for operators are so large. When $3 N>N_{f}>2 N$, then $N_{f}<2 \tilde{N}=$ $2 N_{f}-2 N$. In the ultraviolet, all scalar fields, being weakly-coupled, have dimension close to 1 ; thus $2 \approx \operatorname{dim}[Q \tilde{Q}]=\operatorname{dim}[q \tilde{q}]>\frac{3}{2}>\operatorname{dim} M \approx 1$. But as we saw earlier, $R_{Q}>\frac{1}{2}>R_{q}$ for $N_{f}>2 N$, from which we learn that near the infrared fixed point $\operatorname{dim}[Q \tilde{Q}]=\operatorname{dim} M>\frac{3}{2}>\operatorname{dim}[q \tilde{q}]$. (Note we do not need duality for this conclusion; we need only the anomaly-free Rsymmetries, which we can determine in each of the two theories separately.) So while $M M$ is classically relevant, and $q \tilde{q} q \tilde{q}$ is classically irrelevant, exactly the opposite is true in the infrared; $q \tilde{q} q \tilde{q}$ is a dangerous irrelevant operator (becoming relevant in the infrared) and $M M$ is a harmless relevant operator - the mass term for $M$ is actually infrared irrelevant, and flows to zero! Thus what happens is this: $\eta \rightarrow 0$, as we would expect, but surprisingly $\tilde{\eta} \rightarrow \infty$ and $\hat{\eta} \rightarrow 0$ in the infrared. The A theory flows to $\mathrm{SQCD}$, and the $\mathrm{B}$ theory flows to $\mathrm{SQCD}+\mathrm{M}$ with $W=y M q \tilde{q}$, consistent with Seiberg duality.

Exercise: Show the reverse is true for $N_{f}<2 N ; Q \tilde{Q} Q \tilde{Q}$ is relevant as is the $M M$ mass term. The B theory therefore loses its mesons, and its infrared physics is $S U(\tilde{N})$ SQCD. Show the A theory becomes $S U(N)$ SQCD+M, by 
reversing the process of integrating out. Following Intriligator and Seiberg [3] and Leigh and Strassler [4], introduce massive gauge-singlet auxiliary fields $\mathcal{M}_{r}^{u}$ to break the $(Q \tilde{Q})(Q \tilde{Q})$ superpotential into two terms of the form $\hat{y} \mathcal{M} Q \tilde{Q}+m \mathcal{M}^{2}$. Then show that when $N_{f}<2 N$, the mass term for $\mathcal{M}$ is irrelevant and the resulting A theory is indeed dual to $S U(\tilde{N})$ SQCD. Why is this a legitimate technique? [Hint: Consider the large- $N$ treatment of the $O(N)$ model, or the $C P(N)$ model.]

Not surprisingly, the borderline case $N_{f}=2 N$ is the most interesting of all. But before turning to it, we have a number of other tasks ahead of us.

\subsection{Relevant Operators and the Seiberg-Dual Theory}

Let us consider another important example of a relevant operator by examining a theory with the same field content as Seiberg's SQCD+M theory: again $S U(n)$ SQCD, with chiral superfields $q_{r}$ and $\tilde{q}^{u}$, and gaugeneutral chiral superfields $M$. However, let us take $W=0$. Then the neutral fields $M_{j}^{i}$ are free. They transform under their own, distinct, $U\left(N_{f}\right)_{1} \times U\left(N_{f}\right)_{2}$ symmetry, which is quite separate from the $S U\left(N_{f}\right)_{L} \times S U\left(N_{f}\right)_{R} \times U(1)_{B} \times U(1)_{\mathcal{R}}$ global symmetry group of the $q$, $\tilde{q}$ and gauge superfields. The $M$ fields are completely decoupled from the physics of the fields charged under $S U(n)$. The $S U(n)$ gauge fields and the $q, \tilde{q}$ multiplets form an SQCD theory with $n$ colors and $N_{f}$ flavors, whose renormalization group flow is the same as for the $S U(N)$ SQCD theory considered earlier (indeed we have here simply relabelled the SQCD theory.) As long as $\frac{3}{2} n<N_{f}<3 n$, the theory will reach a Seiberg fixed point at some $g_{*}$. The $M$ fields, meanwhile, are just floating around as a free and independent sector of the conformal theory. Since they have dimension 1 , we should assign them R-charge $2 / 3$.

But now let us consider what happens if we add the operator $W=$ $y M_{u}^{r} q_{r} \tilde{q}^{u}$ to the action, where $y$ is infinitesimal (and $g=g_{*}$ ). First, as we can no longer rotate $M$ and $q, \tilde{q}$ separately, this breaks the global symmetries down to the usual $S U\left(N_{f}\right)_{L} \times S U\left(N_{f}\right)_{R} \times U(1)_{B} \times U(1)_{\mathcal{R}}$. Second, since the requirement of anomaly-freedom fixes the R-charge of $q$ and $\tilde{q}$ at $1-\frac{n}{N_{f}}$, we must assign the R-charge of $M$ to be $2 \frac{n}{N_{f}}$ in order that the $\mathrm{R}$-charge of $W$ be 2 . But this is not consistent with the dimension of $M$, which we know is $1+\operatorname{order}\left(y^{2}\right)$ - and this is a sign that nonzero $y$ is inconsistent with our original $y=0$ fixed point. This means that for small 
and nonzero $y$ the theory cannot be at a fixed point any longer, so a renormalization group flow is going to ensue. But will $y$ grow or shrink? We can compute the beta function for $y$ at extremely small $y$, where we know $\operatorname{dim} M=1$ and $\operatorname{dim} q=\operatorname{dim} \tilde{q}=\frac{3}{2}\left(1-n / N_{f}\right)$ :

$$
\begin{aligned}
\beta_{y} & =-y(3-\operatorname{dim} M-2 \operatorname{dim} q) \\
& =-y\left(3-1-2 \frac{3}{2}\left[1-n / N_{f}\right]\right)=y\left(1-\frac{3 n}{N_{f}}\right)
\end{aligned}
$$

and thus for any $N_{f}<3 n$ this is a relevant operator. Therefore, $y$ grows, and the Seiberg SQCD fixed point is destabilized. This is shown in Fig. 5.

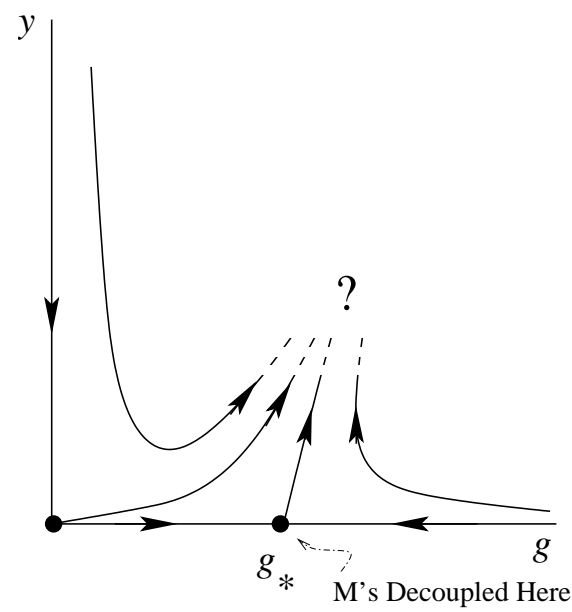

Figure 5. The $M$ fields are decoupled on the $y=0$ axis; the theory reaches a Seiberg SQCD fixed point. If $y \neq 0$, the Seiberg SQCD fixed point is destabilized; what happens next?

What happens next is anyone's guess; but Seiberg is not just anyone, and he guessed something very brilliant. He considered the possibility that the theory with nonzero $y$ does reach a fixed point, as illustrated in Fig. 6 .

Notice this fixed point is stable; no matter what $g$ and $y$ we start with, as long as both are nonzero, we will reach the $g_{*}^{\prime}, y_{*}$ fixed point. Only if $y$ is zero will we reach the fixed point with $g=g_{*}, y=0$; and if only $g$ is zero, the well-known fact that $\phi^{4}$ has positive beta function in four dimensions implies $y$ will flow to zero. (See my TASI 2001 lectures [2] for a 


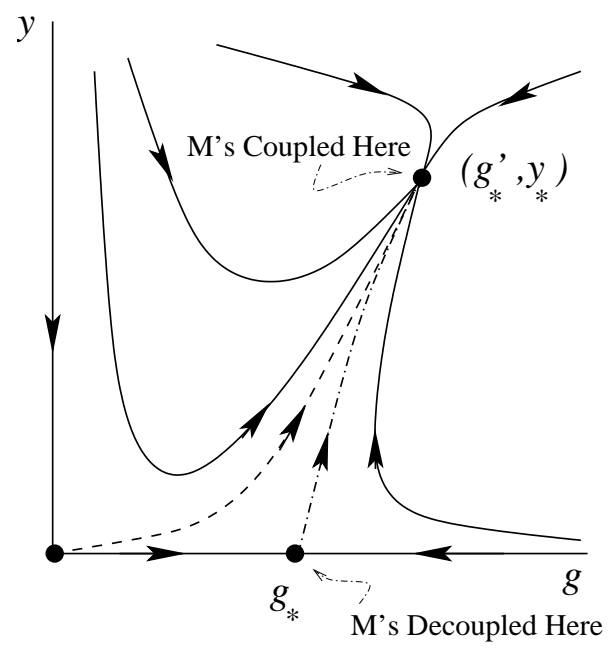

Figure 6. Seiberg's conjecture: if $y \neq 0$, the flow ends at a new fixed point $(g, y)=\left(g_{*}^{\prime}, y_{*}\right)$. The dashed curve is an SQCD+M theory, flowing from a free theory to its fixed point. The dot-dashed curve flows between an SQCD fixed point (with additional free $M$ fields) to an SQCD+M fixed point.

more convincing proof.) This is all consistent with the two beta functions

$$
\beta_{y}=\frac{1}{2} y\left(\gamma_{M}+2 \gamma_{0}\right), \beta_{g} \propto-\left[3 n-N_{f}+N_{f} \gamma_{0}\right]
$$

where now $\gamma_{M}$ and $\gamma_{0}$ are functions of $g$ and $y$. [Note $b_{0}$ is still $3 n-N_{f}$, independent of $y$, since the $M$ fields are gauge-singlets and do not affect the one-loop gauge beta function.] These beta functions both vanish (as they must) when $\operatorname{dim} q=\frac{3}{2} R_{q}$ and $\operatorname{dim} M=\frac{3}{2} R_{M}$; you can work out the $\gamma$ 's for yourself.

Note that on this graph the free fixed point at $g, y=0$ is the ultraviolet fixed point for the SQCD+M theory with $n$ colors, whereas the $g=g_{*}^{\prime}, y=$ $y_{*}$ fixed point is the infrared fixed point for the SQCD+M theory. More precisely, any choice of $g, y$ with $g \neq 0, y \neq 0$ at the ultraviolet cutoff will lead to the same SQCD+M infrared fixed point. However, if one wishes to define the SQCD+M theory in the continuum, with no ultraviolet cutoff, one must take $g, y \rightarrow 0$ as the ultraviolet cutoff is removed; this must be done in a region where $\beta_{y}<0$. The dashed line is an example of a flow from the free fixed point in the infinite ultraviolet to the SQCD+M fixed point in the far infrared. Other flows with the same ultraviolet and infrared fixed 
points exist between the dashed line and the $y=0$ axis. The dot-dashed line represents a different flow, from the Seiberg SQCD fixed point with $n$ colors, plus a decoupled set of $M$ mesons, to the infrared fixed point of $\mathrm{SQCD}+\mathrm{M}$.

Seiberg then noticed that the dimension and global charges of $M_{u}^{r}$ at the $g=g_{*}^{\prime}, y=y_{*}$ fixed point — the infrared fixed point of the SQCD+M theory - are now precisely the same as that of the gauge invariant operator $Q^{r} \tilde{Q}_{u}$ in the $S U(N)$ SQCD gauge theory, where $n=N_{f}-N \equiv \tilde{N}$. This led him to his duality proposal - that the putative fixed point of the $S U(\tilde{N})$ theory with the $M q \tilde{q}$ superpotential is identical to the putative fixed point of the $S U(N)$ SQCD theory with $N_{f}$ flavors $Q, \tilde{Q}$. Other checks (see Intriligator's notes) confirm this idea is consistent. For instance, the baryon operators $[Q]^{N}$ and $[q]^{\tilde{N}}$ nontrivially match; they have the same Rcharges, and hence the same dimensions, and they have the same $S U\left(N_{f}\right)_{L}$ transformation properties (here we see how important it is that $Q^{r}$ and $q_{r}$ are in conjugate representations of $S U\left(N_{f}\right)_{L}$.) $\quad$ Fig. 7 illustrates this suggestion.

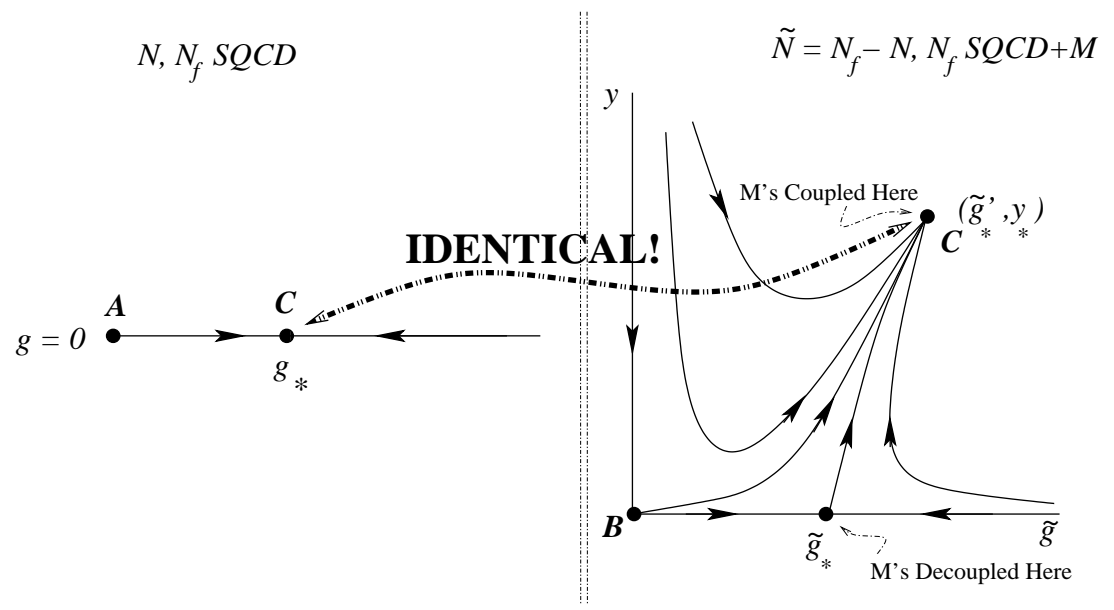

Figure 7. Seiberg's conjecture: the two fixed points marked $C$ are actually identical. The $S U(N)$ SQCD and $S U(\tilde{N})$ SQCD + M theories, which approach points $A$ and $B$ in the ultraviolet, give two different ways to reach this one fixed point marked $C$, and thus provide two different choices of variables for describing it. 


\subsection{Seiberg Duality, Precisely}

We'll come back and discuss this further a bit later on. First, we'd better ask ourselves "what is duality"? Let's examine what Seiberg is telling us.

In the regime of $\frac{3}{2} N<N_{f}<3 N$, Seiberg has given us the following picture of duality. There are two flows, each from a free theory, which approach the same nontrivial infrared conformal fixed point. Let us call the original weakly-coupled ultraviolet theory $\mathrm{A}$, the dual weakly-coupled ultraviolet theory B, and the infrared fixed point which the theories share C. The flow is as shown in Fig. 8. The theories are different in the ultraviolet, and match only in the infrared, approaching each other as the distance scale is taken longer and longer. ${ }^{6}$

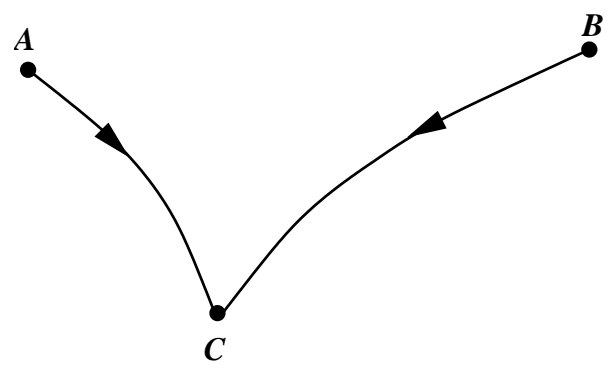

Figure 8. Fixed points A and B can each be perturbed so that they flow to fixed point C.

This is not the only case for which Seiberg duality applies, however. As you saw in Intriligator's lectures, the cases of $N_{f}=N$ and $N_{f}=N+1$ are ones in which there is a dual theory of massless mesons and baryons; for $N_{f}=N$ there is chiral symmetry breaking on the entire moduli space, while for $N_{f}=N+1$ all chiral symmetries are preserved at the origin of the moduli space. In both of these cases the low-energy theory is infrared-free,

\footnotetext{
${ }^{6}$ The flow into an infrared fixed point is always controlled by the least irrelevant operator allowed by symmetries, and thus both flows must approach the fixed point from the direction associated with this operator. However, the two flows might enter the fixed point with a different sign, or phase, for the least irrelevant coupling. Thus the theories may only match in the extreme infrared. With some additional work it is possible to match them more precisely in some circumstances. See for example Kapustin and Strassler [5]. This issue will not arise in the context of "exact Seiberg duality," which we will discuss in great detail below.
} 
and is not well-defined at arbitrarily short distance. Moreover, because the superpotential interactions $W=X \operatorname{det} M+\cdots$ or $W=\operatorname{det} M+\cdots$ are of degree $N+1$, these are perturbatively nonrenormalizable theories for $N>2$, so we cannot even do reliable perturbative calculations. Instead, the meson-baryon theories must be defined with an ultraviolet cutoff $\Lambda_{U V}$ if we are to make any sense of them; and consequently the RG flow now takes the form in Fig. 9. Here, theory $\mathrm{C}$ is a free theory - yet another conformal fixed point - of massless mesons and baryons (since all couplings flow to zero in the extreme infrared.)

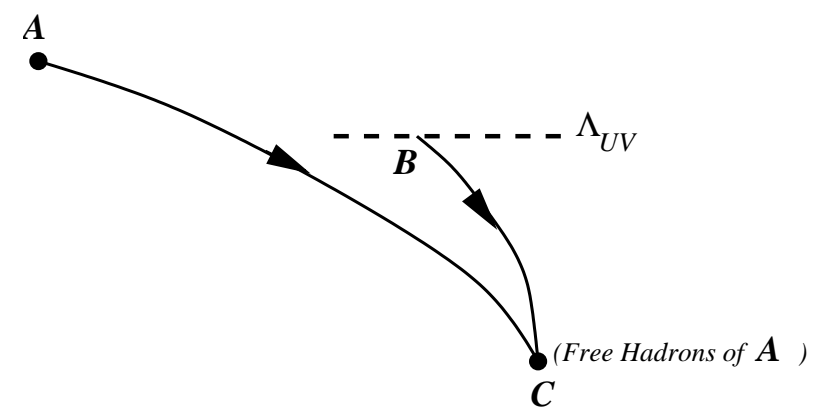

Figure 9. Think of theory A as QCD with massless quarks, theory B as the chiral Lagrangian with its ultraviolet cutoff, and theory $\mathrm{C}$ as the infrared theory of free massless pions; so it is in the case of SQCD with $N_{f}=N$ or $N+1$.

This should look familiar. This is precisely the diagram which one would use for real-world QCD, in the limit where the three light quarks are massless. The theory of massless quarks and gluons becomes strongly coupled at some scale $\Lambda$. A separate theory, known as the linear sigma model, is used to describe mesons ${ }^{7}$ as far as their low-momentum interactions are concerned. This sigma model is non-renormalizable and must be defined with a cutoff, $\Lambda_{U V}$; the theory is ambiguous near this scale, and does not exist above it. Now why do both theories appear in the same textbook? It is because they are believed to have the same infrared physics! The sigma model is known as an "effective theory" for QCD because it provides a simpler and more analytically tractable model for the infrared collective behavior of quarks and gluons. But we wouldn't introduce it if we thought that a

$\overline{7}$ and baryons, as solitons called "skrymions" 
nonperturbative calculation in QCD, using lattice gauge theory, would give different answers for physical questions. The statement that one theory is an effective theory for another is the statement of duality in a particular context: namely, that Theory A and Theory B approach each other in the limit where all external momenta are small, and that the theory they approach (a weakly coupled theory of pions, in this case) has two descriptions, one using the variables of quarks and gluons (and computable using lattice gauge theory) and a second using the variables of pions themselves (computable in sigma-model perturbation theory. $)^{8}$

Seiberg duality also contains one more spectacular generalization of these ideas, known as the free magnetic phase, illustrated in Fig. 10. For $N+1<N_{f} \leq \frac{3}{2} N$, the dual theory of $S U(N)$ is of the same form as described before - it has gauge group $S U(\tilde{N})$, fields $q, \tilde{q}, M$, etc. - but now there is a big difference. The $S U(\tilde{N})$ gauge group has a positive beta function. Consequently, just as with QED with massless electrons, and just as with the sigma model, the coupling constants of the theory run to zero in the far infrared, and the theory must be defined with an ultraviolet cutoff. In some ways, this case is very similar to those with $N_{f}=N$ or $N+1$, and to real-world QCD. But the big difference is that the low-energy effective theory is itself a gauge theory, and the dual degrees of freedom are not hadrons of the original theory, but fractional quasiparticles. For instance, in the particular case of $S U(5)$ with 8 flavors, the baryon operator $Q^{1} Q^{2} Q^{3} Q^{4} Q^{5}$ is not a particle in the low-energy theory; instead, this fiveparticle state of the high-energy theory falls apart into a three-quasiparticle state $q_{6} q_{7} q_{8}$ in the low-energy description as an $S U(3)$ gauge theory, with its 8 flavors of $q$ and $\tilde{q}$ and $64 M_{r}^{u}$ singlets.

Thus Seiberg duality, in its original manifestation, incorporates three different types of relationships, as illustrated in the three figures above. In the conformal window, two asymptotically free gauge theories flow to a single nontrivial conformal field theory. For smaller $N_{f}$, below the conformal window, an asymptotically-free gauge theory and an infrared-free gauge theory with a cutoff flow to the same infrared physics, described best using the variables of the infrared-free gauge theory. And for $N_{f}=N$ and $N+1$,

\footnotetext{
${ }^{8}$ It should perhaps be remarked that some members of our community still see this viewpoint - that the sigma model is "dual" to QCD - as merely giving a fancy name to something very simple, namely effective field theory. But an essential point of these lectures is that effective field theory, when it employs quasi-particles or bound states as effective degrees of freedom, is a special case of something much more general, namely duality.
} 


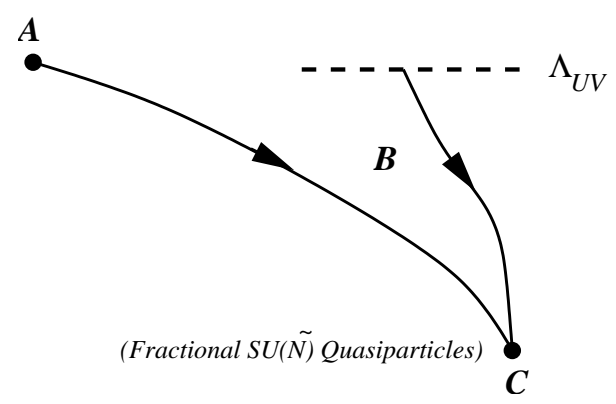

Figure 10. In the free magnetic phase, theory B is a gauge theory with a Landau pole, so it requires a cutoff; theory $\mathrm{C}$ is a free theory of the gauge bosons and quarks of theory B, which are quasiparticles of fractional charge in theory A.

the second theory is an infrared-free theory of chiral superfields - hadrons of the original theory - and again the latter theory well-describes the infrared physics. Note that in each of these cases the far-infrared physics is conformally invariant, although in the last two the fixed point is free (in the dual variables. $)^{9}$

\subsection{Seiberg Duality versus Electric-Magnetic Duality}

The alert student will no doubt be wondering, however, what this type of duality has to do with electric-magnetic duality, and in particular, the duality present in $\mathcal{N}=4$ supersymmetric Yang-Mills theory. In such dualities, there is a coupling constant $g$, or more precisely $\tau$, defined in Eq. (1.8), which is truly constant. Unlike the Seiberg conformal fixed points, which are isolated points $g=g_{*}$ within the space of coupling constants, the $\mathcal{N}=4$ theory has a conformal fixed point for every value of $\tau$. And unlike Seiberg's duality, which is an infrared duality, the electric-magnetic duality of $\mathcal{N}=4$ is supposed to be an exact duality between the theory with $S U(N)$ gauge group and coupling $\tau$ and the theory with $S U(N) / \mathbf{Z}_{N}$ gauge group and coupling $-1 / \tau$. In fact, an entire $S L(2 . \mathbf{Z})$ acts on $\tau$, generated by shifts of the theta angle by $2 \pi(\tau \rightarrow \tau+1)$ and by the electric-magnetic duality transformation $\tau \rightarrow-1 / \tau$.

\footnotetext{
${ }^{9}$ Note the fixed point is approached rapidly in the conformal window, while it is approached logarithmically when the low-energy theories have an infrared-free gauge coupling.
} 
One of the apparent distinctions between these dualities is no distinction at all. Although we phrased Seiberg duality as an "infrared duality," it is nonetheless true that Seiberg duality is exact when applied to the Seiberg conformal fixed points directly. The reason for this is trivial: fixed points are the same in the ultraviolet as they are in the infrared, so if two fixed points match in the infrared, they are the same at all scales. That is, if we take the $S U(N)$ theory with $g=g_{*}$, it is identical to the $S U(\tilde{N})$ theory with $\tilde{g}=\tilde{g}_{*}, y=y_{*}$; both are describing the exactly conformal theory $\mathrm{C}$. So if we look only at the conformal theory C, then Seiberg duality provides two descriptions of a single theory, just as $S U(N) \mathcal{N}=4$ is a single theory with multiple descriptions. I'll have a bit more to say about this later.

On the other hand, the Seiberg fixed point is an isolated point inside the space of coupling constants, whereas there really are an infinite number of $\mathcal{N}=4$ theories, each indexed by a different value of $\tau$. (The $S U(N)$ theory with $\tau=.2 i$ is equivalent to the $S U(N) / \mathbf{Z}_{N}$ theory with $\tau=-(.2 i)^{-1}=5 i$, in that there exists a map between operators of the two theories such that their Green functions are identical; however, the $S U(N)$ theory with $\tau=.3 i$ is a distinct theory from that with $\tau=.2 i$, in that there exists no such map.) As we will soon see, $\mathcal{N}=1$ supersymmetric theories also sometimes have continuously-infinite spaces of conformal field theories, so this kind of phenomenon is not limited to extended supersymmetry. But still, duality is applied to a finite set of theories in SQCD, and to an infinite set of theories in $\mathcal{N}=4$.

Nonetheless, this distinction is not very important. What is important for us here is that in both cases duality acts as a transformation on a space of theories, whatever that space may happen to be. ${ }^{10}$ In $\mathcal{N}=1$ theories with continuous spaces of conformal field theories, there are sometimes dualities which are both of Seiberg type and of electromagnetic type, and in other cases one finds dualities which generalize both Seiberg duality and electric-magnetic duality simultaneously. So the two types of dualities are really just different manifestations of a single phenomenon.

\subsection{Why Seiberg Duality can be Exact, and When}

Let us now see that Seiberg duality is an exact duality, not merely an infrared duality, even in some contexts beyond the purely conformal field

${ }^{10}$ To understand how electric-magnetic duality manifests itself as a change of variables within a path integral, the reader should study the second section of Seiberg and Witten [6], or the pedagogical presentation in my TASI 2001 and Trieste 2001 lectures $[2,7]$. 
theories. This will be very important for us.

We spent some time earlier investigating the precise relationship between the $S U(N)$ theory with $N_{f}$ flavors and its $S U(\tilde{N})$ dual; we saw that the weakly-coupled versions of these theories, which we called A and B, flow to an interacting conformal field theory C. Of course, if this is true for $N, N_{f}, \tilde{N}=N_{f}-N$, then it is also true for $N, N_{f}-1, \tilde{N}-1=\left(N_{f}-1\right)-N$. Let us refer to the $S U(N)$ theory with $N_{f}-1$ flavors as A', and its dual as B'; their infrared fixed point we can call C'. Then A' and B' both flow to $\mathrm{C}^{\prime}$, just as A and B both flow to C.

We can check Seiberg duality by considering flows from A to A', which induce flows from $\mathrm{C}$ to C'. In particular, let us reduce $N_{f}$ by one by adding a mass term $W=m Q^{1} \tilde{Q}_{1}$. In the dual theory $W=y M q \tilde{q}+\hat{m} M_{1}^{1}$, which leads ${ }^{11}$ to the condition $0=\partial W / \partial M_{1}^{1}=y q_{1} \tilde{q}^{1}+\hat{m}$. Therefore $\left\langle q_{1} \tilde{q}^{1}\right\rangle \neq 0$, breaking the $S U(\tilde{N})$ gauge group to $S U(\tilde{N}-1)$. The massive gauge bosons absorb the fields $q_{1}$ and $\tilde{q}_{1}$, leaving $N_{f}-1$ flavors. Thus theory B indeed flows to theory B', the dual of A'.

But now we have found an important consequence, illustrated in Fig. 11. If $m$ is very large, much larger than the strong coupling scale $\Lambda_{A}$, then the flow of $\mathrm{A}$ is of the form $\mathrm{A} \rightarrow \mathrm{A}^{\prime} \rightarrow \mathrm{C}^{\prime}$. Similarly, in this regime $\mathrm{B}$ flows to C' by way of B'. But suppose instead that $m$ is extremely small. Then the flow is of the form $\mathrm{A} \rightarrow \mathrm{C} \rightarrow \mathrm{C}^{\prime}$, while that of $\mathrm{B}$ is $\mathrm{B} \rightarrow \mathrm{C} \rightarrow \mathrm{C}^{\prime}$. What we obtain in this case, then, is two descriptions of a single flow from $\mathrm{C}$ to C'. We can now dispense with the ultraviolet starting points A and B, by taking the strong-coupling scale $\Lambda_{A}$ to infinity, and similarly for $\Lambda_{B}$, while holding $m$ fixed. This leaves us, in this limit, with a single flow from $\mathrm{C}$ to C', described from two different points of view. And thus we see that although we began with an "infrared duality," which was between theories $\mathrm{A}$ and $\mathrm{B}$, and involved two different theories flowing to the same infrared fixed point, the existence and consistency of this duality implies inevitably that we must also have "exact dualities" as well, in which we have two descriptions, identical at all length scales, of one and only one nontrivial renormalization group flow.

So if anyone ever tells you that "duality is only an infrared effect in field theory, while in string theory duality is exact," tell them to read this chapter! (And to read a paper by Kapustin and myself [5], from 1999, in which we worked out more complex and complete three-dimensional examples in great detail.) As we will see, this point undergirds the duality cascade.

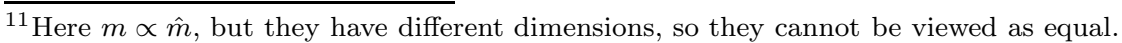



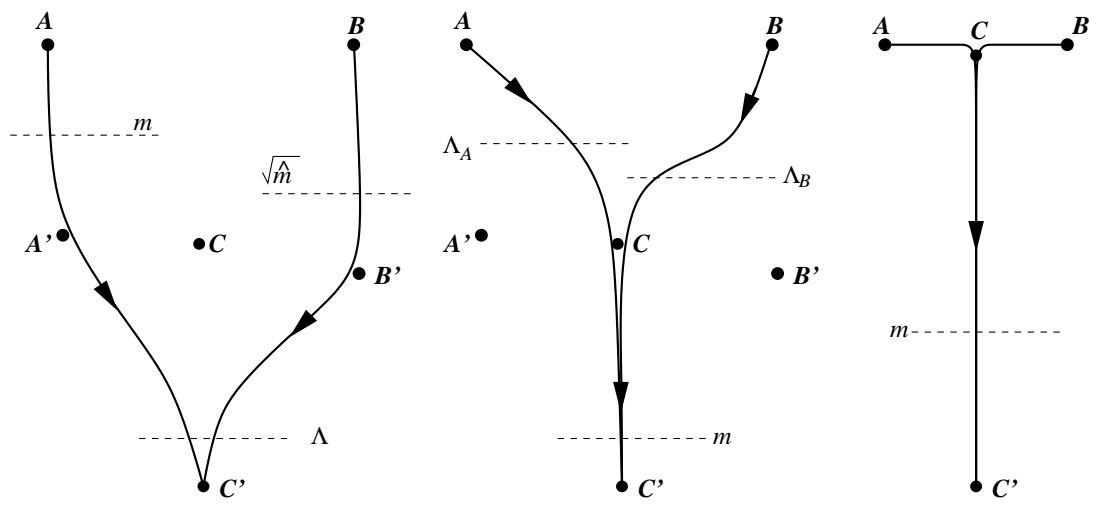

Figure 11. Theories $A$ and $B$ both flow to $C$ in the infrared; if perturbed by $m$ to $A^{\prime}$ and $B^{\prime}$, they both flow to $C^{\prime}$. If $m$ is large the theories flow near to $A^{\prime}$ and $B^{\prime}$; if $m$ is small they flow close to $C$. By scaling $\Lambda_{A} \rightarrow \infty$ and $\Lambda_{B} \rightarrow \infty$, holding $m$ fixed, we obtain two descriptions of the flow from $C$ to $C^{\prime}$ generated when $C$ is perturbed by $m$.

Let us see how this comes into our discussion of quartic operators, using Fig. 12. We saw that if $\frac{3}{2} N<N_{f}<2 N$ then the Seiberg fixed point of SQCD has quartic $Q \tilde{Q} Q \tilde{Q}$ operators which are relevant. In these cases, the coupling $\eta$ grows in the infrared. Similarly, the meson mass term is a relevant coupling $M M$ at the dual SQCD $+\mathrm{M}$ fixed point, which means the quartic operator $q \tilde{q} q \tilde{q}$ is irrelevant: $\tilde{\eta}$ shrinks in the infrared. If we now consider a flow in which $\eta$ is initially very small, then the SQCD theory will flow from A (a free theory) to $\mathrm{C}$ (the Seiberg fixed point perturbed very slightly by $\eta$ ) to C' (a fixed point where $\eta \rightarrow \infty$.) This is shown in the left side of Fig. 12. On the right side of the figure, we see the dual theory will flow from B (a different free theory) to $\mathrm{C}$ (the same intermediate fixed point, though described in $S U(\tilde{N})$ variables, slightly perturbed by a meson mass term, or equivalently large $\tilde{\eta}$ ) to C' (the same final fixed point, in dual variables, where $\tilde{\eta} \rightarrow 0$.) This is important. The flow from the Seiberg fixed point of $S U(N) S Q C D$ and $N_{f}$ flavors with $\eta \ll 1$ to the theory of $\eta \gg 1$ is exactly Seiberg dual to the flow from the $S U(\tilde{N}) S Q C D+M$ with small meson mass $(\tilde{\eta} \gg 1)$ to the $S U(\tilde{N}) S Q C D$ fixed point with $\tilde{\eta} \ll 1$. As illustrated in Fig. 12, we thereby obtain two descriptions of one physical theory, the theory which flows from an ultraviolet fixed point at $\mathrm{C}$ to an infrared fixed point at C'. 


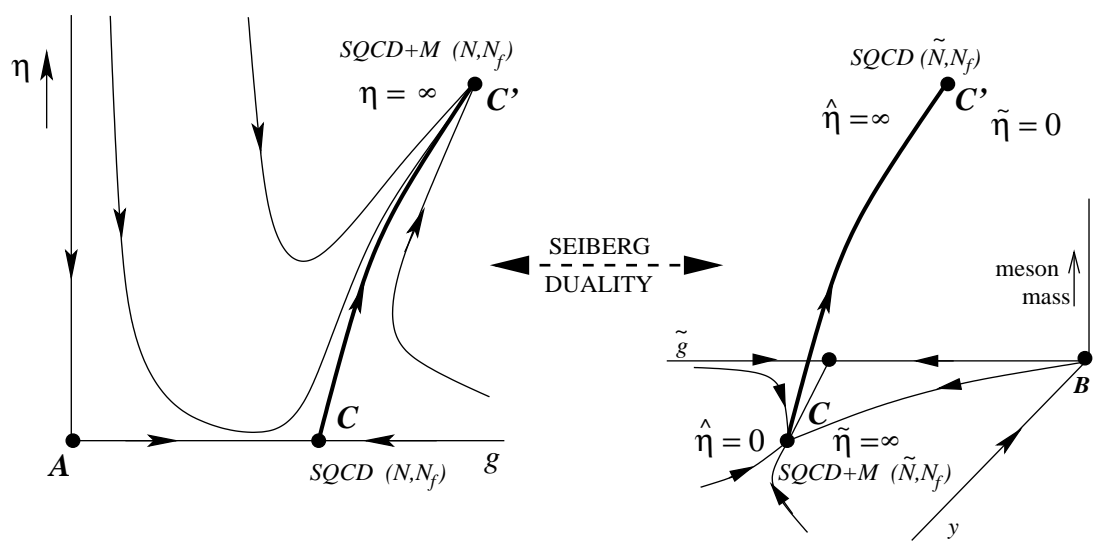

Figure 12. The flow on the left, in which the quartic coupling $\eta$ flows from 0 to $\infty$, is exactly Seiberg-dual to the flow on the right, in which the meson mass coupling $\hat{\eta}$ also flows from 0 to $\infty$, or equivalently the dual quartic coupling $\tilde{\eta}$ flows from $\infty$ to 0 . Theory $C$ can be reached from either theory $A$ or theory $B$, but only along the solid line from $C$ to $C^{\prime}$ is Seiberg-duality exact.

Before continuing, please make sure you understand this point, and Fig. 12, fully. It takes some serious thought, but it is absolutely crucial for an understanding of why the duality cascade is exact Seiberg duality in action.

\subsection{Exactly Marginal Operators}

We now turn to marginal couplings, and the special circumstances in supersymmetric theories that sometimes make them exactly marginal.

Let's argue that $\mathcal{N}=4$ Yang-Mills is finite. Consider an $\mathcal{N}=1$ gauge theory with three chiral superfields $\Phi_{i}$ in the adjoint representation, and a superpotential $W=\sqrt{2} y \operatorname{tr} \Phi_{1}\left[\Phi_{2}, \Phi_{3}\right]$. I will use canonical normalization here for the $\Phi_{n}$, so $y=g$ gives the $\mathcal{N}=4$ supersymmetric theory. But let's not assume that $y=g$. For any $g, y$, the symmetry relating the three fields ensures they all have the same anomalous dimension $\gamma_{0}$, which is a single function of two couplings. The beta functions for the couplings are

$$
\beta_{y}=\frac{3}{2} y \gamma_{0} ; \beta_{g}=\frac{-g^{3}}{16 \pi^{2}} \frac{3 N \gamma_{0}}{1-g^{2} N / 8 \pi^{2}}
$$

These are proportional to one another, so the conditions for a fixed point $\left(\beta_{y}=0\right.$ and $\left.\beta_{g}=0\right)$ reduce to a single equation, $\gamma_{0}(g, y)=0$. But this is 
one equation on two variables, so if a solution exists, it will (generically) be a part of a one-dimensional space of such solutions.

Now, does a solution exist? For small $g, y$, we know that $\gamma_{0}(g, y=0)<0$ and that $\gamma_{0}(g=0, y)>0$; so yes, by continuity, there must be a curve, passing through $g=y=0$, along which $\gamma_{0}=0$ and $\beta_{y}=\beta_{g}=0$ (and thus perturbation theory has no infinities along this line.) The renormalization group flow must look like the graph in Fig. 13. Both the theory with $y=0$ and the theory with $g=0$ are infrared free; yet a set of nontrivial field theories lies between. Notice that we do not know the precise position of the curve $\gamma_{0}=0$; in particular, we have not shown that $g=y$ gives $\gamma_{0}=0$. However, the existence of a finite theory (and one which is renormalizationgroup infrared stable against perturbations) only requires arguments using $\mathcal{N}=1$ supersymmetry. Of course, since the theory at $g=y$ has more symmetry (namely $\mathcal{N}=4$ ) it is natural to expect $g=y$ to be the solution to $\gamma_{0}(g, y)=0$.

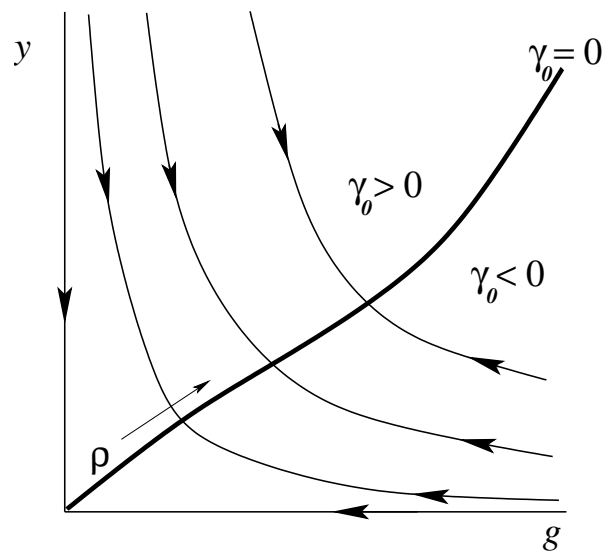

Figure 13. In some $\mathcal{N}=1$ theories one can argue for a line of fixed points indexed by an exactly marginal coupling $\rho$; perturbation theory has no divergences on this line. Only in $\mathcal{N}=4$ is the equation for this line $g=y$.

The motivation for introducing this $\mathcal{N}=1$-based reasoning is there are many $\mathcal{N}=1$ field theories which are also finite, as one can show using similar arguments. For example, replace the $\mathcal{N}=4$ superpotential with $W=y \operatorname{tr} \Phi_{1}\left\{\Phi_{2}, \Phi_{3}\right\}$; the discussion is almost unchanged, except that $g=y$ is not the solution to $\gamma_{0}=0$. Another simple example is $S U(3)$ with $N_{f}=9$ 
and the superpotential

$$
W=y\left[Q^{1} Q^{2} Q^{3}+Q^{4} Q^{5} Q^{6}+Q^{7} Q^{8} Q^{9}+(Q \rightarrow \tilde{Q})\right]
$$

for which

$$
\beta_{y}=\frac{3}{2} y \gamma_{Q} ; \beta_{g} \propto(9-9)+9 \gamma_{Q} .
$$

The existence of these theories was discovered in the 1980s; the slick proof presented above is in Leigh and Strassler (1995).

The coupling which parametrizes the line of conformal fixed points (which is actually a complex line, since the couplings are complex) is called an "exactly marginal coupling," and the operator which it multiplies is called an "exactly marginal operator." Let's call this complex coupling $\rho$. (In the $\mathcal{N}=4$ case we can identify $\rho$ as equal to the gauge coupling $i / \tau$, but in a more general $\mathcal{N}=1$ finite theory these will not be simply related.) Unlike $\lambda$ in $\lambda \phi^{4}$, which is marginal at $\lambda=0$ but irrelevant at $\lambda \neq 0, \rho$ is marginal at $\rho=0$, and remains marginal for any value of $\rho$. Thus $\rho$ is a truly dimensionless coupling, indexing a continuous class of scale-invariant theories. It can be shown ${ }^{12}$ that there is always a holomorphic version of $\rho$. Moreover, it is very common for such classes of theories to be acted upon by duality transformations. In fact, for $\mathcal{N}=4$, electric-magnetic duality (S-duality) acts on this coupling $\rho$ as in Fig. 14, identifying those theories at large $\rho$ with those at small $\rho$. (Strictly speaking, the duality relates the $S U(N)$ theory with the $S U(N) / \mathbf{Z}_{N}$ theory; these have the same local dynamics, but have slightly different behavior for subtle questions beyond the scope of these lectures.)

One more example of a finite theory: $\mathcal{N}=2 S U(N)$ Yang-Mills with $N_{f}=2 N$. This theory is just like $\mathcal{N}=1 N_{f}=2 N$ SQCD except for the presence of a chiral superfield $\Phi$ in the adjoint, and the superpotential

$$
W=f \tilde{Q}_{r} \Phi Q^{r} .
$$

The $S U\left(N_{f}\right)$ global symmetry and charge conjugation symmetry assure all $Q, \tilde{Q}$ fields have the same $\gamma$. Because

$\beta_{f}=\frac{1}{2} f\left[\gamma_{\Phi}+2 \gamma_{0}\right], \beta_{g} \propto-\left[3 N-N\left(1-\gamma_{\Phi}\right)-2 N\left(1-\gamma_{0}\right)\right]=-N\left[\gamma_{\Phi}+2 \gamma_{0}\right]$

we again have a finite theory with a marginal coupling $\rho=i / \tau$ passing through $g=0, f=0$. (As in $\mathcal{N}=4$, we know this coupling is acted on by a duality transformation that takes $\tau \rightarrow-1 / \tau$.)

\footnotetext{
${ }^{12}$ In theories with exactly marginal operators, one can always find a dimensionless holomorphic invariant which is neutral under all continuous global symmetries $[3,4,8,9]$.
} 


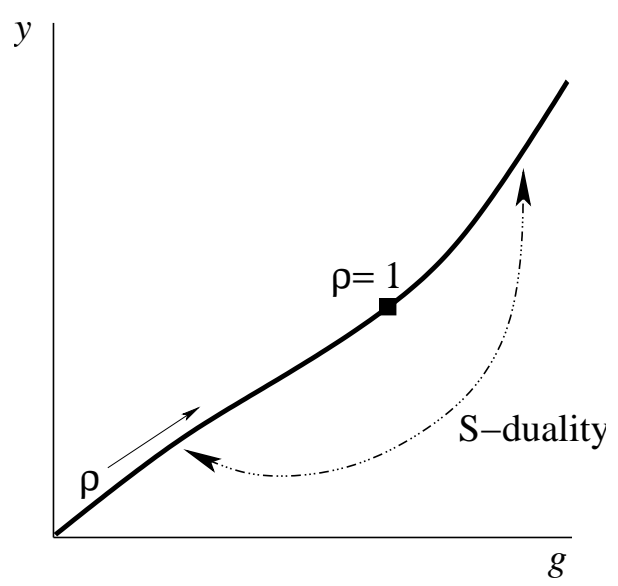

Figure 14. The action of S-duality on the line of fixed points.

Exercise: Suppose only $k$ of the fields are coupled to $\Phi$. In this case, those $Q$ 's which are coupled to $\Phi$ have one anomalous dimension, and those that aren't have a different one. Show that there is no marginal operator in this theory.

Exercise: Suppose that $k$ of the fields have one coupling $f$ and $N-k$ have a nonzero coupling $f^{\prime}$; this breaks $\mathcal{N}=2$ supersymmetry to $\mathcal{N}=1$. Argue that $f-f^{\prime}$ flows to zero and $\mathcal{N}=2$ is restored at low energy.

Now let us return to our discussion of Sec. 1.4. We consider the theory of $\mathcal{N}=1 \mathrm{SQCD}$ with $N_{f}=2 N$, and add the quartic superpotential (1.13)

$$
W=h\left(Q^{r} \tilde{Q}_{u}\right)\left(Q^{u} \tilde{Q}_{r}\right) .
$$

The symmetries ensure that all the $Q$ and $\tilde{Q}$ have the same anomalous dimension $\gamma_{0}$, which is a function of the gauge coupling $g$ and the coupling $\eta=h \mu$. Classically, the gauge coupling is scale-invariant, but recall that $\beta_{\eta}=+\eta$, since the quartic superpotential is classically irrelevant. Quantum mechanically, the beta functions have the form

$$
\beta_{\eta}=\eta\left[1+2 \gamma_{0}\right] ; \beta_{8 \pi^{2} / g^{2}} \propto 3 N-2 N\left(1-\gamma_{0}\right)=N+2 N \gamma_{0}
$$

and thus we see that $\beta_{\eta} \propto \beta_{g}$. This means that, as before, the conditions 
for a fixed point to exist, namely $\beta_{\eta}=0=\beta_{g}$, reduce (for nonzero $g$ and $\eta)$ to a single condition,

$$
1+2 \gamma_{0}=0 \Rightarrow \gamma_{0}=-\frac{1}{2}
$$

Again, this is one condition on two couplings, so any solution will generically be part of a one-dimensional space of solutions. Seiberg has already convinced us that it is likely that a solution does exist for $h=0$; if $W=0$, this theory is SQCD in the conformal window, and we expect it has a fixed point $g=g_{*}$ where $\gamma_{0}\left(g_{*}\right)=-\frac{1}{2}$. Even though we know nothing about the curve $\gamma_{0}(g, h)=-\frac{1}{2}$, the very existence of the Seiberg fixed point then implies that a continuous space of fixed points emanates from this point, as in Fig. $15 .{ }^{13}$

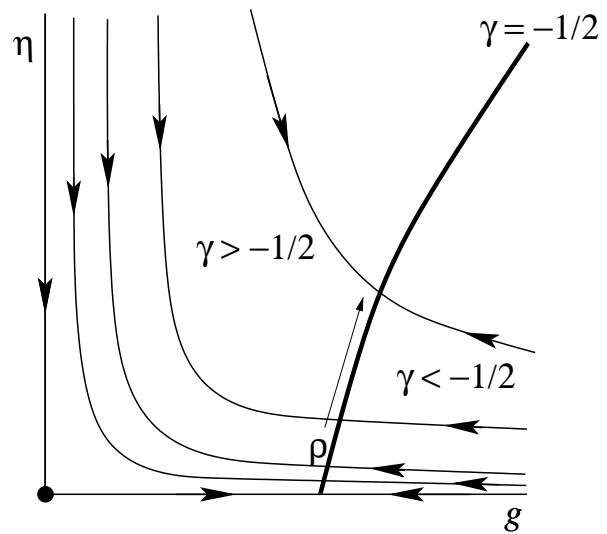

Figure 15. In the $\mathcal{N}=1$ theory of $S U(N)$ with $2 N_{f}$ flavors and a quartic superpotential, $\eta$ is marginal at $g=g_{*}$ and there is a line of fixed points emerging from the fixed point at $(g, \eta)=\left(g_{*}, 0\right)$.

So here the classically irrelevant operator has been converted into an

\footnotetext{
${ }^{13}$ The curve $\gamma_{0}=-\frac{1}{2}$ drawn here, with the property that $d h / d g>0$, is an educated guess. The guess is based on the fact that superpotential couplings tend to give positive contributions to anomalous dimensions, therefore requiring a larger gauge coupling to cancel them and bring $\gamma_{0}$ back to $-\frac{1}{2}$. We will use this guess throughout in our schematic discussions. However, if this guess is wrong, the only effect is to require a minor redraw of the figures. The main results of these lectures are all independent of the precise shape of this curve.
} 
exactly marginal one! The coupling $\rho$ which parametrizes the line of fixed points, and on which duality symmetries might act, now has nothing to do with the gauge coupling. In fact, $\rho=0$ corresponds to $g=g_{*}$, not $g=0$. Granted, the choice of coordinate $\rho$ is not unique. But no matter what coordinate we choose, we see that nowhere are these conformal field theories near $g=\eta=0$. We therefore have no hope of seeing them appear in any perturbation expansion. (Nonetheless, we will be able to see them in another weakly-coupled limit, namely that of supergravity.)

But now consider how Seiberg duality acts on these theories. The SQCD $+\mathrm{M}$ dual has $\tilde{N}=N$ colors, but it is not the same as the original theory; it has $W=y M q \tilde{q}$ when $h=0$. When $h \neq 0$ one must add $\hat{h} M M$ to the superpotential, as before, but now $\hat{h}$ is marginal. The beta functions near the Seiberg fixed point with $(\tilde{g}, y, \hat{h})=\left(\tilde{g}_{*}^{\prime}, y_{*}, 0\right)$ take the form

$$
\beta_{\hat{h}}=\hat{h}\left[-1+\gamma_{M}\right], \beta_{y}=\frac{1}{2} y\left[\gamma_{M}+2 \gamma_{0}\right], \beta_{\tilde{g}} \propto-N\left[1+2 \gamma_{0}\right] .
$$

Thus a fixed point requires $\gamma_{M}=1$ and $\gamma_{0}=-\frac{1}{2}$, namely two conditions on three couplings, meaning that the generic solution is a one-dimensional manifold of fixed points. Since the conditions are satisfied at the $\hat{h}=0$ fixed point, a line of conformal field theories extends from that point. But of course it must! The $N_{f}=2 N$ SQCD and $N_{f}=2 N$ SQCD + M theories are dual, so in the infrared they have the same physics. If a line of conformal fixed points ends on the fixed point of one, a similar line must end on the fixed point of the other. This is just as in Fig. 12, except in this case the theories marked $C$ and $C^{\prime}$ are connected by a line of conformal fixed points instead of a renormalization-group flow.

In the presence of a marginal mass term, it is not obvious whether we should or should not integrate out $M$, but suppose we do. Then, by the analysis we did earlier for $N_{f} \neq 2 N$, we find the SQCD+M theory, having lost its $M$ fields, becomes SQCD with a quartic superpotential $q \tilde{q} q \tilde{q}$, with a physical coupling $\tilde{\eta}$. Thus we have a duality between two theories of identical form, with the same gauge group and the same matter content and the same quartic superpotentials. The only difference between them is that the exactly marginal coupling of one is roughly the inverse of the other. Moving away from the $\eta=0$ fixed point, with $\eta$ increasing from zero, is the same as moving away from the dual fixed point with the dual coupling $\tilde{\eta}$ decreasing from infinity. Conversely, as we increase $\eta$ to infinity, we expect $\tilde{\eta}$ will decrease to zero. In fact, we can guess that there should be an exactly marginal coupling $\rho$, whose exact relation to $g, \eta$ we do not know 
(and which, indeed, is not uniquely specified) but which has the property that $\rho=0$ corresponds to $\eta=0, \rho=\infty$ corresponds to $\tilde{\eta}=0$, and under $\rho \rightarrow 1 / \rho$, the theory is self-dual [3, 4]! The Seiberg fixed points sit at $\rho=0, \infty$, and Seiberg-duality has been generalized to something that more closely resembles the electric-magnetic duality of $\mathcal{N}=4$ Yang-Mills.

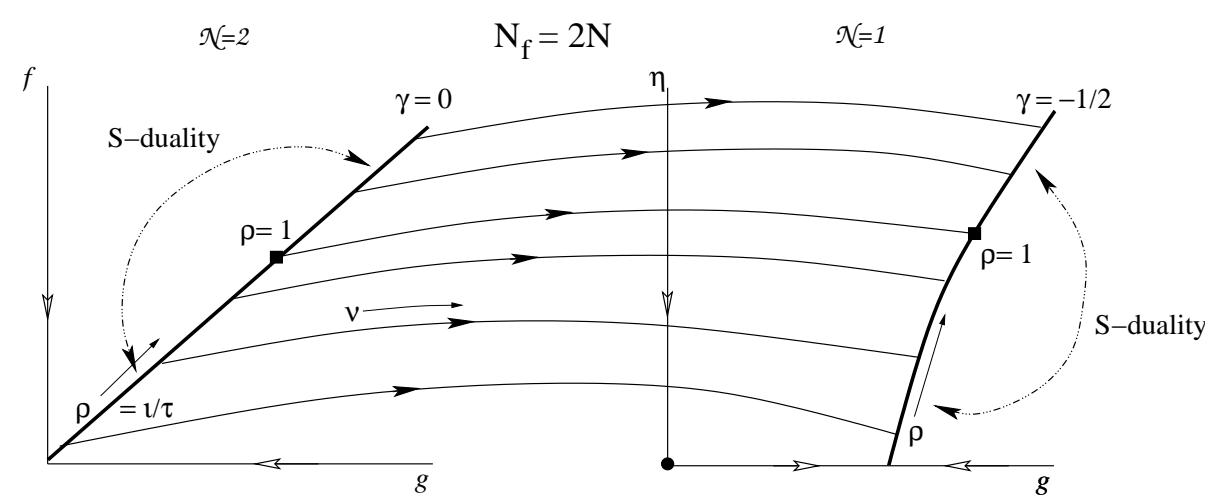

Figure 16. The $\mathcal{N}=1$ theory with $N_{f}=2 N$ inherits S-duality from the finite $\mathcal{N}=2$ theory with $N_{f}=2 N$; when the latter is perturbed by a mass $m_{\Phi}$ for the adjoint $\Phi$, the ensuing flow along $\nu=m_{\Phi} / \mu$ carries it to the former in the infrared. Note the S-duality of the low-energy theories is Seiberg-duality in this case.

Now, since the theory is nonrenormalizable, we probably should say something about the ultraviolet. We know a perfectly good ultraviolet theory into which we can embed this theory, namely the finite $\mathcal{N}=2$ gauge theory with $N_{f}=2 N$ discussed above, perturbed by a mass term $m_{\Phi} \Phi^{2}$ for the adjoint chiral superfield. When we integrate out $\Phi$, we obtain ${ }^{14}$ a quartic operator $(Q \tilde{Q})(Q \tilde{Q})$ with coefficient $h=-f^{2} / 4 m_{\Phi}$. Thus the endpoint of the flow is one of the $\mathcal{N}=1 N_{f}=2 N$ conformal theories we were just discussing. It is natural to expect that each $\mathcal{N}=2$ field theory

${ }^{14} \mathrm{I}$ am glossing over a subtlety: when the quartic operator is rewritten as a product of two gauge-invariant bilinears $Q \tilde{Q}$, the precise flavor structure of the operator is very slightly different from the one given in Eq. (1.13). We could fix this if there were time, but it would require too much of a detour. Moreover, the subtlety is not present for the conifold theory, which we will study in the next section. The interested reader can examine the last section of Leigh and Strassler [4] where the precise operator is obtained, and can adjust the discussion of this section accordingly. 
flows to a unique $\mathcal{N}=1$ conformal field theory, along a flow which looks schematically like Fig. 16. In the figure, the flow is parameterized by $\nu=$ $m_{\Phi} / \mu$. We thereby obtain a one-to-one map between finite $\mathcal{N}=2$ theories, indexed by the $\mathcal{N}=2$ gauge coupling $\tau$, and our $\mathcal{N}=1$ conformal theories, indexed by a holomorphic coupling $\rho$. Since $\rho$ is an arbitrary coordinate, we can replace it by $i / \tau$, if we wish - but be careful to remember that this " $\tau$," while it is the inverse of the gauge coupling in the $\mathcal{N}=2$ theory, is not the inverse gauge coupling of the $\mathcal{N}=1$ theory, as is clear from the graph!

Note one more nice thing about this picture. The $\mathcal{N}=2$ theories have an electromagnetic duality $\tau \rightarrow-1 / \tau$. The manifold of $\mathcal{N}=1$ fixed points is acted on by Seiberg duality. Clearly, in this case, the electromagnetic duality of the high-energy theory descends to Seiberg duality of the lowenergy theory!

\subsection{Seiberg Duality with a Quartic Operator}

In summary, in the presence of quartic operators Seiberg duality becomes even more elegant than before. We have seen that $S U(N)$ SQCD with $N_{f}$ flavors near its Seiberg fixed point and with a quartic coupling $\eta$ is dual to a theory of exactly the same form, except with $\tilde{N}$ colors and with a quartic coupling $\tilde{\eta} \sim 1 / \eta$. Usually $\eta$ flows either to zero or to infinity, and $\tilde{\eta}$ flows to the reverse. For $N_{f}>2 N, \eta$ is irrelevant and $\eta=0$ is stable. But, importantly for these lectures, when $N_{f}<2 N, \eta$ grows in the infrared and as in Fig. 12, the flow from $\eta=0$ to $\eta=\infty$ is exactly Seiberg dual to the flow from $\tilde{\eta}=\infty$ to $\tilde{\eta}=0$.

Finally, the case $N_{f}=2 N$ is very special. The theory is self-dual, $\eta$ is exactly marginal, and Seiberg duality acts to invert $\eta$, or more precisely to invert some suitable holomorphic coupling $\rho(\eta)$ (Fig. 17.)

Let us conclude with an analogy that helps us to summarize these results. We may attempt to think about the beta functions, and the figures which describe how the theory moves within the space of coupling constants, in terms of a model dynamical system. With the caveat that it has not been proven that this is legitimate, let us attempt to think metaphorically of the flow of a theory to the infrared in terms of a ball rolling in a potential, subject to high friction. The friction is necessary because the beta function equations are first-order differential equations, not second-order. Then the renormalization group flows in Figs. 13, 15 and 17 are associated with a ball rolling in a potential which has an absolutely-flat valley bottom (the manifold of conformal fixed points, where all beta functions vanish) surrounded 


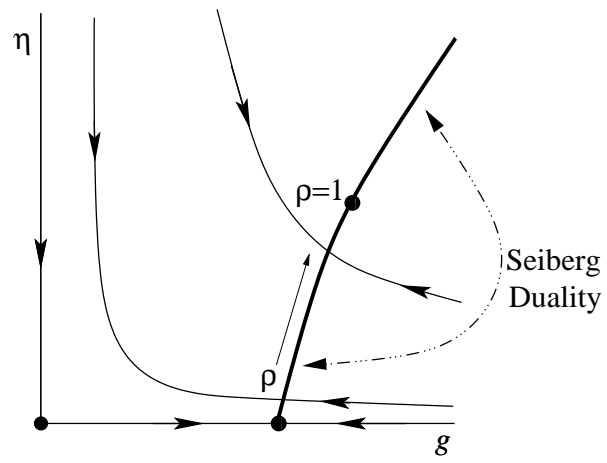

Figure 17. Seiberg duality acts on the space of fixed points of Fig. 15.

by steep rising walls on either side (which drive all flows into the valley bottom.) If you put the ball anywhere in the space, it will flow (subject to high friction) into the valley; once there, it feels no potential gradient, and comes to a stop. By contrast, consider Fig. 4, or the left-hand diagram in Fig. 12, for SQCD with $N_{f}<2 N$. Here, the valley (the line connecting $\mathrm{C}$ and C') is still surrounded by steep hillsides (since all flows approach this line) but the valley also has a tilt (which drives all flows away from $\mathrm{C}$ and toward C'). All generic flows therefore end at C', even if they initially flow toward $\mathrm{C}$. We will find this metaphor of the ball rolling in a landscape very useful below, so keep it in mind.

\section{The Conifold Theory}

We now turn to a simple generalization of the model with $N_{f}=2 N$ and a quartic superpotential. We will gauge an $S U(N)$ subgroup of the $S U(2 N)$ flavor symmetry, and we will also alter the operator slightly to enhance the global symmetries. It is this theory which appears when we study D3-branes on the conifold. The theory first arose in the context of Type IIA and M-Theory brane constructions, but it was first investigated in the AdS/CFT context, and thereby popularized, by Klebanov and Witten [10]. We will consequently refer to it as the "Klebanov-Witten model."

\subsection{The Field Theory}

The model of interest has $S U(N) \times S U(N)$ gauge group, two chiral superfields $A_{1}, A_{2}$ in the $(\mathbf{N}, \overline{\mathbf{N}})$ representation (called the "bifundamental 
representation") of the gauge group, and two chiral superfields $B_{1}, B_{2}$ in the $(\overline{\mathbf{N}}, \mathbf{N})$ "anti-bifundamental" representation. It has an $S U(2)_{L} \times$ $S U(2)_{R} \times U(1)_{B}$ symmetry, where the $A_{r}$ transform under the first factor, the $B_{u}$ under the second, and both transform under baryon number with charge $\pm 1 / N$. Finally, we will add a superpotential which preserves the full $S U(2) \times S U(2) \times U(1):$

$$
W=h \operatorname{tr} \operatorname{det}_{r, u}\left(A_{r} B_{u}\right)
$$

where the trace is necessary now because $A$ and $B$ both carry two gauge indices, one for each group, and thus should be viewed as matrices. Explicitly, letting $\alpha$ be a group index in the first $S U(N)$ gauge group and $a$ be a group index in the second, this can be written as

$$
W=h\left[\left(A_{1}\right)_{a}^{\alpha}\left(B_{1}\right)_{\beta}^{a}\left(A_{2}\right)_{b}^{\beta}\left(B_{2}\right)_{\alpha}^{b}-\left(A_{1}\right)_{a}^{\alpha}\left(B_{2}\right)_{\beta}^{a}\left(A_{2}\right)_{b}^{\beta}\left(B_{1}\right)_{\alpha}^{b}\right] .
$$

This theory has not one but two exactly marginal coupling constants. ${ }^{15}$ For each of the two gauge groups, the number of "flavors" contributing to the one-loop beta function is $2 N$ ( $N$ from $A_{1}$ and $B_{1}$ and $N$ from $A_{2}$ and $\left.B_{2}\right)$ so $b_{0}=3 N-2 N=N$ for both groups. The beta functions for the two gauge couplings $g_{1}, g_{2}$ (and the corresponding 't Hooft couplings) and for the coupling $\eta=h \mu$ are

$$
\begin{aligned}
\beta_{g_{1}} & =-\frac{g_{1}^{3}}{16 \pi^{2}} \frac{N+2 N \gamma_{0}}{1-\frac{g_{1}^{2} N}{8 \pi^{2}}} \Rightarrow \beta_{\lambda_{1}}=-\frac{\lambda_{1}^{2}}{8 \pi^{2}} \frac{1+2 \gamma_{0}}{1-\frac{\lambda_{1}}{8 \pi^{2}}}, \\
\beta_{g_{2}} & =-\frac{g_{2}^{3}}{16 \pi^{2}} \frac{N+2 N \gamma_{0}}{1-\frac{g_{2}^{2} N}{8 \pi^{2}}} \Rightarrow \beta_{\lambda_{2}}=-\frac{\lambda_{2}^{2}}{8 \pi^{2}} \frac{1+2 \gamma_{0}}{1-\frac{\lambda_{2}}{8 \pi^{2}}}, \\
\beta_{\eta} & =\eta\left(1+2 \gamma_{0}\right),
\end{aligned}
$$

where all four charged fields have the same anomalous dimension $\gamma_{0}\left(g_{1}, g_{2}, h\right)$ because of the global symmetries (including chargeconjugation.) Since the condition for a fixed point clearly puts one condition on three couplings (namely $\gamma_{0}=-\frac{1}{2}$ ), any nontrivial fixed point is part of a two-dimensional complex manifold of fixed points, parametrized by two exactly marginal couplings. And we know solutions to these conditions exist, since when $g_{2}=0$ and $h=0$ the theory becomes $S U(N)$ SQCD with $N_{f}=2 N$, which has a Seiberg fixed point with $\gamma_{0}=-\frac{1}{2}$.

It is useful to draw a picture representing where this two-complexdimensional space of fixed points sits within the space of three complex

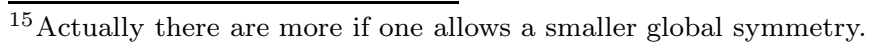


couplings. Suppressing the phase of $h$ and the imaginary part of the $g_{i}$, we can draw the manifold, somewhat schematically (since we don't know its exact equation), as in Fig. 18.

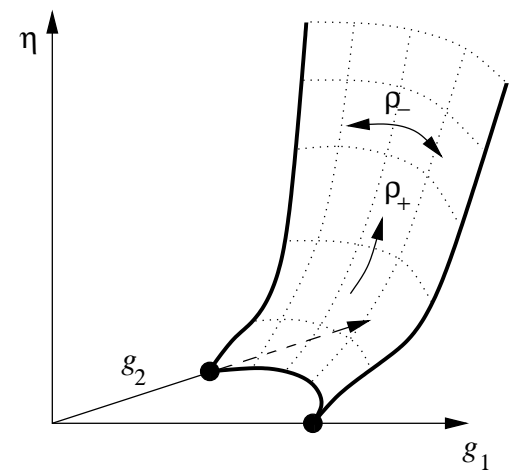

Figure 18. The two-dimensional space of $\mathcal{N}=1$ conformal theories inside the three-dimensional space of couplings. The exact shape of the manifold is unknown, but inessential.

Of course the manifold is symmetric under $g_{1} \leftrightarrow g_{2}$. Note that $\beta_{g_{1}} \propto$ $\beta_{g_{2}}$, which means there is a line of interesting conformal fixed points lying in the $h=0$ plane and connecting the two Seiberg fixed points at $\left(g_{1}, g_{2}\right)=$ $\left(g_{*}, 0\right)$ and $\left(g_{1}, g_{2}\right)=\left(0, g_{*}\right)$. When $g_{2}=0$ (or $\left.g_{1}=0\right)$, the theory becomes an $N_{f}=2 N$ SQCD model with a quartic operator, of the sort we studied earlier (though the quartic operator is very slightly different). For this reason, the intersection of the manifold of fixed points with the $g_{2}=0$ plane looks very similar to Fig. 15.

Fig. 15 also reminds us that this manifold is infrared stable. The signs of the beta functions are such that from any point in coupling constant space that is off the manifold of fixed points, the flow into the infrared will bring us onto the manifold at some particular fixed point. Our analogy of a ball rolling in a potential applies here too; the valley is now two-dimensional, but otherwise is similar to what we discussed at the end of Sec. 1.10. We have an valley, with a perfectly flat floor, surrounded by high, steeply rising walls. The ball will always flow down to the valley floor, and once there, it will come to a stop.

It is very natural to parameterize this manifold of fixed points using two 


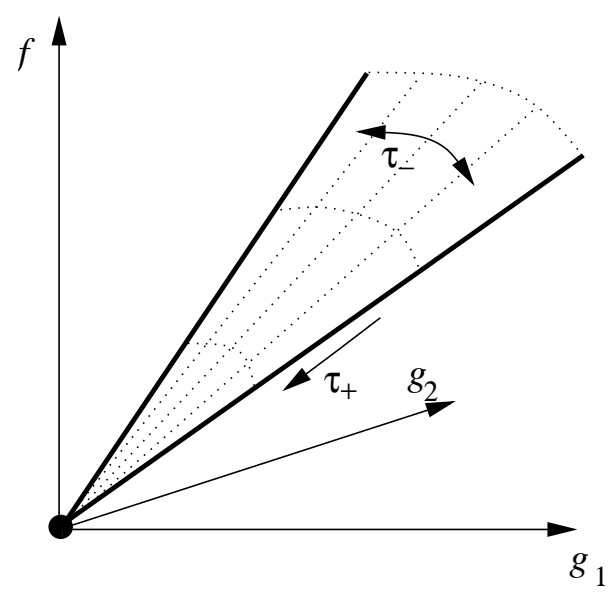

Figure 19. The two-dimensional space of $\mathcal{N}=2$ conformal theories inside the three-dimensional space of couplings. The dark lines lie in the $g_{2}=0$ and $g_{1}=0$ planes.

complex parameters $\rho_{+}$and $\rho_{-}$that are symmetric and antisymmetric in $g_{1} \leftrightarrow g_{2}$. We can do this as we did in the $N_{f}=2 N$ SQCD model, by finding a finite $\mathcal{N}=2$ field theory, with its own dualities, to serve as an ultraviolet definition of the theory. There is indeed such a model, given by adding to the Klebanov-Witten theory the superfields $\Phi$ in the adjoint of the first $S U(N)$ and $\phi$ in the adjoint of the second. The $\mathcal{N}=2$ superpotential, a simple generalization of (1.20), is $W=f \operatorname{tr}\left[A_{r} \Phi B^{r}+B_{r} \phi A^{r}\right]$. Notice the flavor group contains only a single $S U(2)$, since the flavor indices of $A$ and $B$ are contracted (although there is a second hidden $S U(2)$, an Rsymmetry associated with the $\mathcal{N}=2$ supersymmetry.) This theory which corresponds to D3-branes sitting on a $\mathbf{Z}_{2}$ orbifold, by the way - is finite and has two marginal couplings $\tau_{1}$ and $\tau_{2}$, corresponding to its gauge couplings - or at least, that is the correspondence at large imaginary $\tau_{i}$. The manifold of fixed points is shown in Fig. 19. As we did before, we can choose $\tau_{1}$ and $\tau_{2}$ to parametrize the manifold, or even better, we can use $\tau_{ \pm}=\tau_{1} \pm \tau_{2}$. Although it was known from the finite $\mathcal{N}=2$ theories with a single gauge group that this $\mathcal{N}=2$ theory is acted on by duality transformations of $\tau_{+}$, such as $\tau_{+} \rightarrow-1 / \tau_{+}$, it was not until Witten constructed this theory using branes [11] that it was demonstrated that the dualities include also $\tau_{-} \rightarrow \tau_{-}+2 \tau_{+}$. 
Exercise: Show this $\mathcal{N}=2$ theory has two marginal couplings, using the beta function arguments given earlier.

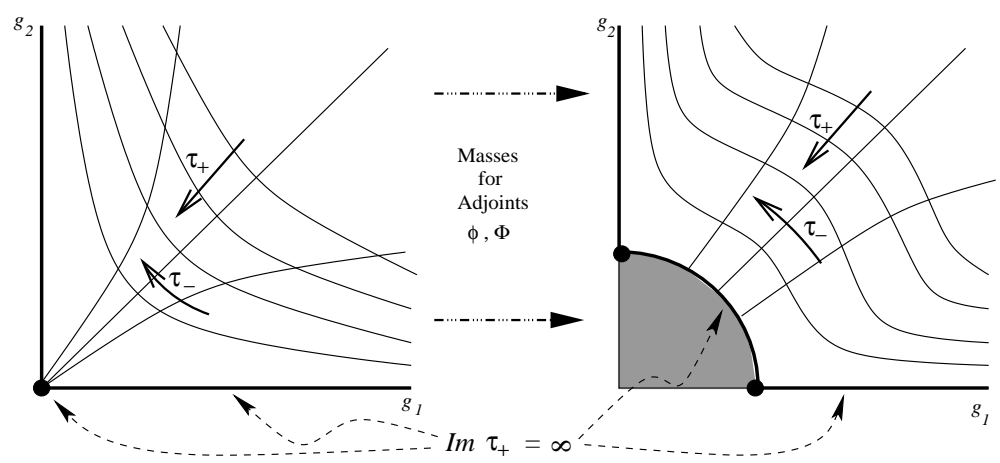

Figure 20. On the left the two-dimensional space of $\mathcal{N}=2$ conformal theories projected onto the $g_{1}, g_{2}$ plane; on the right, the same for the $\mathcal{N}=1$ KlebanovWitten model. The space of theories on the left, naturally parameterized by $\tau_{ \pm}$, flows under perturbation by masses to the space of theories on the right. This flow allows the use of $\tau_{ \pm}$as coordinates on the space of Klebanov-Witten conformal theories. At the thick solid boundaries, $\tau_{+} \rightarrow i \infty$. Note there are no Klebanov-Witten fixed points near $g_{1}=g_{2}=0$.

We proceed as we did in Sec. 1.9: by adding masses for $\Phi$ and for $\phi$ with opposite $\operatorname{sign}\left(m_{\Phi}=-m_{\phi}\right)$, we can obtain the Klebanov-Witten theory in the infrared. The two-dimensional manifold of $\mathcal{N}=2$ fixed points shown in Fig. 19 flows down, in a simple generalization of Fig. 16, to the twodimensional manifold of $\mathcal{N}=1$ fixed points shown in Fig. 18. We can choose $\tau_{ \pm}$as the coordinates of the latter, in lieu of $\rho_{ \pm}$. This is indicated schematically in Fig. 20. In the left graph, the manifold of fixed points in Fig. 19 has been projected down onto that $g_{1}, g_{2}$ plane, with the coordinate $\eta$ suppressed; the coordinates $\tau_{ \pm}=4 \pi / g_{1}^{2} \pm 4 \pi / g_{2}^{2}$ here. In the right graph, to which the left graph flows (as in Fig. 16) when the adjoint fields of the $\mathcal{N}=2$ theory are given masses, the manifold of fixed points in Fig. 18 has similarly been projected down onto its own $g_{1}, g_{2}$ plane, with the coordinate $\eta$ suppressed. In this case $\tau_{ \pm}$are not simply related to the gauge couplings of the $\mathcal{N}=1$ gauge theory. Instead, the $\mathcal{N}=1$ theory on the right with labels $\tau_{+}, \tau_{-}$is the low-energy limit of the $\mathcal{N}=2$ theory on the left with 
gauge couplings $\tau_{+}, \tau_{-}$and adjoint mass terms.

In the Klebanov-Witten model, $\tau_{-}$still has some connection with the difference between the two $\mathcal{N}=1$ gauge couplings, since it vanishes when $g_{1}=g_{2}$; thus for small $g_{1}-g_{2}$ we can expect $\tau_{-} \propto \frac{4 \pi i}{g_{1}^{2}}-\frac{4 \pi i}{g_{2}^{2}}$. But this is not true when the difference of the gauge couplings is large, as is clear from Figs. 18 and 20; in some places $\tau_{-}$varies along with $\eta$. Meanwhile $\tau_{+}$ in general has little to do with the sum of gauge couplings; in some places it reaches $i \infty$ at finite values of $\frac{1}{g_{1}^{2}}+\frac{1}{g_{2}^{2}}$. This is to be expected when a complicated two-dimensional manifold is embedded in three dimensions; the coordinates $\tau_{ \pm}$natural on the manifold need not have any simple relation to the coordinates $g_{1}, g_{2}, \eta$ of the space in which it is embedded.

Exercise: Using a classical analysis, show that when $\Phi$ and $\phi$ have masses of opposite sign, the Klebanov-Witten theory emerges in the infrared. What happens if the masses are not in the ratio -1 ?

Finally, and crucially, we can expect the $\mathcal{N}=2$ duality transformations on $\tau_{ \pm}$will be inherited by the Klebanov-Witten theory, generalizing Fig. 16 . Indeed Witten's brane-based arguments [11], properly supplemented, show that they are. The $S L(2, \mathbf{Z})$ duality $\tau_{+} \rightarrow-1 / \tau_{+}$acts on both gauge groups symmetrically. On the other hand, the transformation $\tau_{-} \rightarrow \tau_{-}+2 \tau_{+}$does something stranger. To see what it is, we need to compare Fig. 16 to Fig. 20, and Fig. 17 to Fig. 18. In both cases, the first figure represents the second in the $g_{2}=0$ plane. (The slight difference in the shapes is not meant to be meaningful; we don't know the shapes exactly anyway.) What does the transformation $\tau_{-} \rightarrow \tau_{-}+2 \tau+$ mean near the $g_{2}=0$ plane? Comparing the $g_{2}=0$ axis in the right-hand diagram of Fig. 20 to the picture in Fig. 17, we see that a shift of $\tau_{-}$is related to changing $\eta$ from small to large - in short, to motion from one region of the manifold of fixed points to a second region related to the first by Seiberg duality of the first gauge group. And indeed, that's what this duality transformation is: Seiberg duality of one gauge group with the other gauge group fixed. The theory can easily be seen to be invariant under this transformation.

Exercise: Show that the Klebanov-Witten model is invariant in form if you perform Seiberg duality on one of the two gauge groups. You cannot prove $\tau_{-} \rightarrow \tau_{-}+2 \tau_{+}$using the methods I've given you; instead, you should read Witten's paper on the matter [11] 


\subsection{D3-branes on the Conifold}

What makes this particular set of conformal field theories so interesting is that they are easily embedded into string theory, yet (unlike $\mathcal{N}=4$ YangMills) they do not contain a free theory, and as such do not share many properties with free theories. In particular, in contrast to $\mathcal{N}=4$ YangMills, where all chiral superfields have vanishing anomalous dimensions, the chiral superfields here have $\gamma_{0}=-\frac{1}{2}$. How does a weakly-coupled string theory manage to incorporate such a theory?

To answer this question, we need to study the singular complex manifold known as the "conifold." As we will see, just as $\mathcal{N}=4$ Yang-Mills theory is realized as the low-energy dynamics of D3-branes placed in flat space, the Klebanov-Witten theory can be similarly found on D3-branes placed on the conifold. In particular, the branes will sit in a ten-dimensional space consisting of four-dimensional Minkowski space $x^{0}, x^{1}, x^{2}, x^{3}$, to which they lie parallel, and a conifold in the remaining directions.

The conifold, a six-real-dimensional singular space, can be partially defined by embedding it as a three-complex-dimensional space inside four complex dimensions. We choose four complex coordinates $z_{1}, z_{2}, z_{3}, z_{4}$, and define the three-dimensional space using a single complex condition

$$
z_{1}^{2}+z_{2}^{2}+z_{3}^{2}+z_{4}^{2}=0 .
$$

Notice it is not $\left|z_{i}\right|^{2}$ which appears here! Alternatively, we may define the space by using coordinates

$$
\tilde{z}_{r u}=z_{i}\left(\sigma^{i}\right)_{r u}+i z_{4}\left(\mathbf{1}_{r u}\right)
$$

where the $\sigma^{i}$ are the Pauli matrices and $\mathbf{1}$ is a two-by-two unit matrix; then the conifold is defined as

$$
\operatorname{det} \tilde{z}=0 \text {. }
$$

The symmetries on this space are $S O(4) \approx S U(2) \times S U(2)$ (which act on the $i$ and $r, u$ indices respectively) along with a $U(1)$ which rotates all of the $z_{i}$ by the same phase. The space is singular when all $z_{i}=0$. This is shown schematically in Fig. 21.

I have not fully defined the space yet, because I have given only its complex structure and not its metric; for now I will leave the metric unspecified. But let us see what we can learn about its topological and algebraic features. First, the defining equation (2.4) for the space has an obvious 


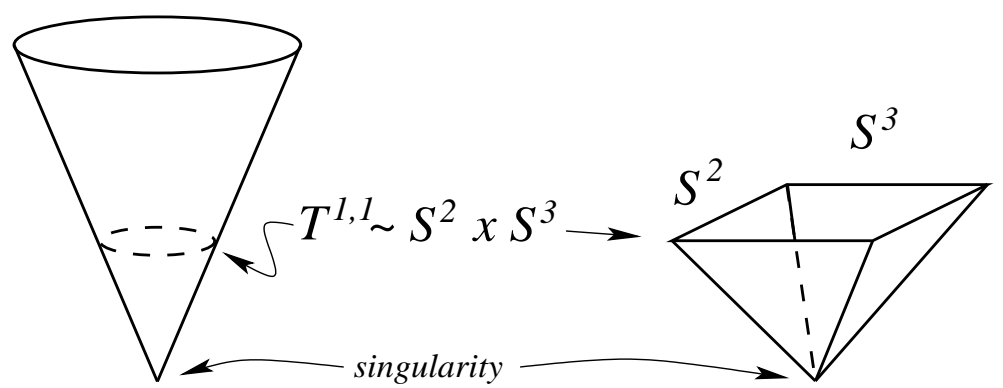

Figure 21. Two schematic views of the conifold as a cone with the space $T^{1,1}$ as its sections and with a singular point at the origin. The diagram at left highlights the smoothness of the space away from the origin; that at right emphasizes that $T^{1,1}$ is topologically $S^{2} \times S^{3}$, with the radii of both spheres shrinking to zero at the origin.

rescaling symmetry $z_{i} \rightarrow \alpha z_{i}$, where $\alpha$ is any positive definite constant. Let us therefore consider the intersection of this space with the additional condition $\sum_{i}\left|z_{i}\right|^{2}=r^{2}, r$ a constant, which removes this symmetry. What does the resulting five-real-dimensional space, which goes by the name $T^{11}$, look like? It is shown in Candelas and de la Ossa [12] that this space is topologically $S^{2} \times S^{3}$. We can see this fairly easily. Let $z_{i}=x_{i}+i y_{i}$; then our space is defined by

$$
\sum_{i} x_{i} y_{i}=0 ; \sum x_{i}^{2}=\sum y_{i}^{2}=r^{2} / 2 .
$$

The solution to this equation is to take $x_{i}$ to be a four-dimensional real vector of length $r / \sqrt{2}$, which parametrizes a 3 -sphere, and then to take $y_{i}$ to be a four-dimensional real vector of length $r / \sqrt{2}$ lying orthogonal to $x_{i}$, which parametrizes a 2 -sphere. Clearly the nature of the 2 -sphere is $x_{i}$-dependent, which makes this an $S^{2}$ fibration over an $S^{3}$; however the fibration is trivial in the end, as Candelas and de la Ossa demonstrate. These authors also show how to define $T^{11}$ as the coset space $[S U(2) \times$ $S U(2)] / U(1)$ (note each $S U(2)$ is an $S^{3}$ and $U(1)$ is an $S^{1}$, so the coset is indeed five-real-dimensional) and how to write an appropriate metric on this space which preserves these symmetries. ${ }^{16}$

${ }^{16}$ Do not get confused between the two $S U(2)$ factors which are symmetries of the space and the two $S U(2)$ factors which can be used to define the space! An analogy is a twosphere: the symmetries which act on it are $S O(3) \approx S U(2) / \mathbf{Z}_{2}$ but the space itself is 
A standard technique in string theory is to "probe" a manifold by placing a D-brane on it, as in Fig. 22, and examining the low-energy field theory of the modes localized on the brane. This "D-brane gauge theory" has a moduli space of vacua which corresponds to the configuration space of the D-brane on the manifold. In the cases where the D-brane can be positioned, stably, at any point on the manifold, the moduli space of the gauge theory and the manifold should precisely be equal. And if $k$ identical D-branes are placed on the manifold, then the D-brane gauge theory's moduli space should consist of $k$ copies of the manifold, up to identifications due to the fact that the $k$ branes are identical.

The simplest example of this is to probe flat space: four-dimensional Minkowski space times a six-dimensional Euclidean space $\mathbf{R}^{6}$. The D-brane gauge theory for a single D3-brane placed in flat space is a four-dimensional $U(1) \mathcal{N}=4$ supersymmetric gauge theory. This theory has six real scalar fields, which have no potential energy constraining their expectation values. Consequently the moduli space is indeed $\mathbf{R}^{6}$. If $k$ D3-branes are placed on the flat space, then the resulting $U(k) \mathcal{N}=4$ theory has six scalars which are $k \times k$ matrices. The $\mathrm{D}$-term and $\mathrm{F}$-term conditions of the gauge theory force these matrices to be diagonal, which means that only the $6 k$ eigenvalues of these matrices can be nonzero. There are no other constraints, so the moduli space is almost $\mathbf{R}^{6 k}$, the configuration space of $k$ points in $\mathbf{R}^{6}$. However, the D3-branes are identical, so the moduli space is reduced by permutations of one D3-brane with another; similarly, in the gauge theory, the eigenvalues can be permuted by Weyl transformations in the $U(k)$ gauge group.

If we place a single D3-brane on the conifold, the moduli space of the D3-brane gauge theory should be the conifold. What gauge theory would give this result? The solution turns out to be a $U(1) \times U(1)$ gauge theory with four charged fields, $A_{1}$ and $A_{2}$ of charge $(1,-1)$ and $B_{1}$ and $B_{2}$ of charge $(-1,1)$. The superpotential is zero. We will now confirm that this theory's moduli space is the conifold.

What is the space of vacua of this theory? There are no F-term conditions, since the superpotential vanishes. The $\mathrm{D}$-term conditions of the two $U$ (1) factors are both of the form $\left|A_{1}\right|^{2}+\left|A_{2}\right|^{2}=\left|B_{1}\right|^{2}+\left|B_{2}\right|^{2}$. We may also use gauge invariance to set the phase of any one of the four fields to zero, making it real, or to set, say, $A_{1}$ and $B_{1}$ to have the same phase. This leaves a total of six independent degrees of freedom out of the original eight

$\overline{\text { the coset } S O(3) / S O(2) \equiv S U(2) / U}(1)$. 
real scalars, so we should have a six-real-dimensional moduli space. How do we characterize it?

We can appeal to a slick argument, that in any supersymmetric gauge theory the solution to the D-term equations (subject to gauge equivalence) is always given by expressing the moduli space in terms of expectation values of holomorphic gauge-invariant operators. The gauge-invariant objects which are functions of $A_{r}, B_{u}$, and not of $A^{\dagger}, B^{\dagger}$, are the four complex bilinears $Z_{r u}=A_{r} B_{u}$; there are no others. But these four bilinears are not independent. In this abelian theory, the chiral superfields are not matrices; they commute, and thus $\left(A_{1} B_{1}\right)\left(A_{2} B_{2}\right)=\left(A_{1} B_{2}\right)\left(A_{2} B_{1}\right)$. This condition may be written $\operatorname{det} Z_{r u}=0$. And this is precisely the defining condition (2.5) of the conifold, written as a three-dimensional complex space embedded in the four-complex-dimensional space of the $Z_{r u}$. Since we have already checked that the moduli space should have six real parameters, there cannot be any additional constraints on the moduli space. Thus our demonstration is complete: this theory's moduli space is a conifold.

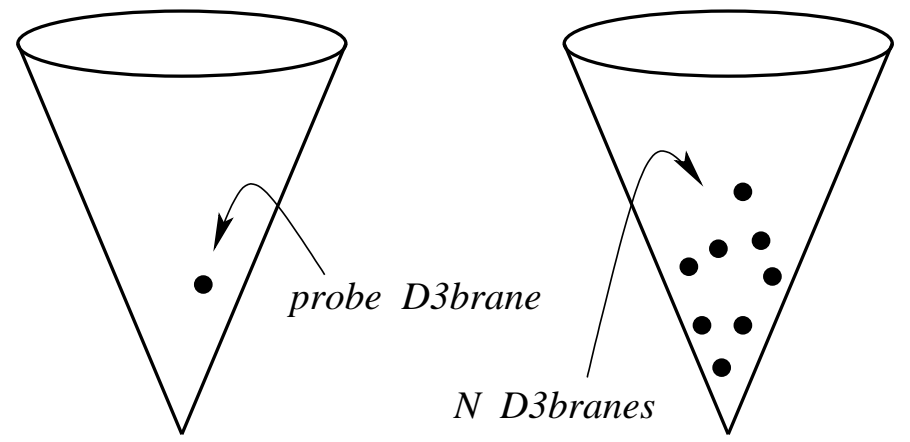

Figure 22. At left, a single D3-brane placed on the conifold serves to probe its structure; at right, $N$ D3-branes are placed on the conifold.

If we place $N$ D3-branes on the conifold, the generalization is a bit more involved. The gauge group becomes $U(N) \times U(N)$; the fields $A_{r}$ and $B_{u}$ are now in the bifundamental and anti-bifundamental representations; and the superpotential is nonzero and takes the by now familiar form

$$
W=h \operatorname{tr} \operatorname{det}_{r, u}\left(A_{r} B_{u}\right) .
$$

This is the theory discussed in the previous section (except that its gauge 
group was $S U(N) \times S U(N)$, a difference we will address shortly.) The conditions for supersymmetric vacua can be written

$$
0=B_{2} A_{r} B_{1}-B_{1} A_{r} B_{2}, 0=A_{2} B_{u} A_{1}-A_{1} B_{u} A_{2} .
$$

Notice these are $N \times N$ complex matrix conditions. Combined with the Dterms, it can be shown (with a small amount of work) that these equations are solved if and only if $A_{r}, B_{u}$ can be simultaneously diagonalized,

$$
A_{r}=\operatorname{diag}\left[a_{r}^{(1)}, a_{r}^{(2)}, \ldots, a_{r}^{(N)}\right], B_{u}=\operatorname{diag}\left[b_{u}^{(1)}, b_{u}^{(2)}, \ldots, b_{u}^{(N)}\right] .
$$

Since it is a trivial identity that

$$
\operatorname{det}_{r, u} a_{r}^{(\sigma)} b_{u}^{(\sigma)}=a_{1}^{(\sigma)} b_{1}^{(\sigma)} a_{2}^{(\sigma)} b_{2}^{(\sigma)}-a_{1}^{(\sigma)} b_{2}^{(\sigma)} a_{2}^{(\sigma)} b_{1}^{(\sigma)}=0
$$

valid for all $\sigma=1, \ldots, N$, it follows that $\operatorname{det}_{r, u} A_{r} B_{u}=0$, and that the moduli space of the $4 N$ complex eigenvalues is $N$ copies of the conifold, up to permutations of the eigenvalues which are gauge-equivalent. This is the same as the configuration space for $N$ indistinguishable D3-branes on the conifold.

The analysis just performed was purely classical. However, the $U(1)$ factors are quantum mechanically problematic. The diagonal $U(1)$ factor is actually decoupled from everything else in the low-energy limit; it has no renormalizable interactions. For this reason, we can truly remove it from our discussions; it makes no contribution to the interesting physics. The other linear combination of the $U(1)$ factors, under which $A$ and $B$ are oppositely charged, does couple, but its beta function is positive and of order $N$, from loops of $A$ and $B$ fields. Consequently it seems it will have a Landau pole in the ultraviolet - unless, of course, its coupling constant is actually zero. The correct interpretation is not immediately obvious, but it does turn out to be true that the coupling constant is zero. As a result, this $U(1)$ actually is not gauged in the quantum theory. Instead, this $U(1)$ is a global symmetry - a true symmetry naturally called "baryon number" - in the quantum theory.

Now, that's fine as far as the moduli space is concerned; if all the branes are away from the origin of the moduli space, then the gauge group is broken to a smaller subgroup (or set of subgroups) and in each subgroup the gauge theory is $\mathcal{N}=4$ Yang-Mills. For instance, suppose we just have one D3brane and we allow $A_{1} B_{1}$ to have an expectation value, so that the D-brane sits at some point away from the singular point of the conifold. Then the gauge group is broken to $U(1)$, and six scalars remain massless - the six possible translations of the D3-brane away from its initial point - exactly 
the number needed to fill out an $\mathcal{N}=4 U(1)$ vector multiplet. I leave the $U(N) \times U(N)$ case to you as an exercise.

Exercise: Show that if all $N$ D3-branes sit at the same nonsingular point, the low-energy theory is $S U(N) \mathcal{N}=4$ Yang-Mills theory. Then show that if the branes are all moved slightly apart, one obtains $N-1$ copies of $U(1)$ $\mathcal{N}=4$ Yang-Mills.

But what happens if the branes all sit at the singularity? Then the details of the singularity are important. It may appear as though we can analyze the singularity gradually by getting closer and closer to it on the moduli space. But this is misleading, and doesn't work. Away from the origin of moduli space, at least one scalar field has an expectation value - call it $v$ - so the lightest massive particles have physical masses $g v$, as shown in Fig. 23. The analysis on the moduli space is valid at energies low compared with all of these masses: $E \ll g v$. But at the origin of moduli space $v \rightarrow 0$, where the singularity lies, $g v$ is also going to zero, so all of the physics lies above the scale $g v$ in this limit. In other words, to understand the physics at the origin of moduli space, we actually want to know what is happening for energies $E \gg g v$ as we take the $v \rightarrow 0$ limit. For this purpose, all the low-energy information about the moduli space is useless. In other words, the order of limits matters; the limit $E \rightarrow 0$ does not commute with the limit $v \rightarrow 0$.

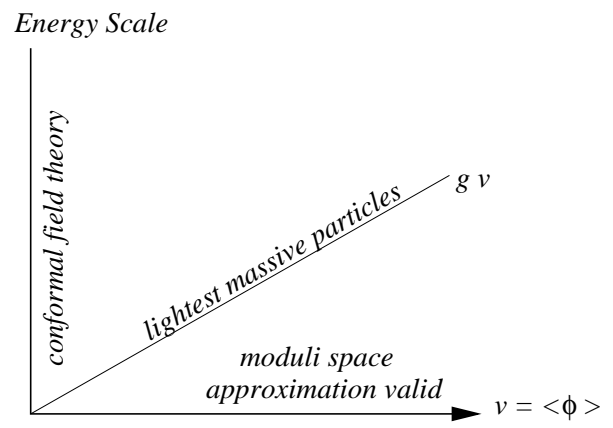

Figure 23. For given $v$, nonzero particle masses are at least $g v$. The conformal field theory is found at all energies when $v \rightarrow 0$, while the moduli space involves the massless fields at low energies for fixed nonzero $v$. 
That's actually fortunate, because there is a profound difference between the field theory on the moduli space and the field theory at the singularity. If, as in the exercise above, all $N$ D3-branes are sitting away from the origin, the low-energy theory is $\mathcal{N}=4$ Yang-Mills, a theory which has no anomalous dimensions $\left(\gamma_{0}=0\right)$ for its chiral superfields. But when all branes sit at the origin, we expect the conformal field theory that we studied earlier, for which the anomalous dimensions for $A_{r}, B_{u}$ are $\gamma_{0}=-\frac{1}{2}$. So these two regimes are really very far apart, and there is no way to interpolate between them easily.

Exercise: Argue that one can use the theory at the singularity to study the low-energy theory on the moduli space, but not vice versa; explain the connection with the irreversibility of renormalization "group" transformations.

How does the theory of D3-branes sitting on the conifold's singularity know that the superfields $A_{r}$ and $B_{u}$ have anomalous dimensions? The metric of the space, when expressed in terms of $A_{r}$ and $B_{u}$, requires it; we will see this in a moment when we write down the Maldacena limit. Another way to see it is to look carefully at the definition of the R-charge. We won't explore this carefully for lack of time.

\subsection{The Maldacena Limit}

Now we want to obtain the string theoretic dual to this gauge theory, in a regime where supergravity is a good approximation. So let us take $N \gg 1$, with the string coupling $g_{s} \ll 1$, holding $g_{s} N \gg 1$ fixed, while accounting for the back-reaction of the branes on the space. We will take the lowenergy limit of the theory on the world-volume of the branes [13], and take the corresponding limit in the ambient spacetime by rescaling the radial coordinate $r$, the distance from the conifold singularity, in the correct way $[14,15]$. In the case of D3-branes in flat space, or on orbifolds of flat space, the effect of this limit was (1) to leave the five angular directions transverse to the D3-branes unchanged, with the same metric they had before, but with a fixed radius $R=\left(4 \pi g_{s} N\right)^{1 / 4}$, and (2) to combine the Minkowski spacetime directions with the radial direction $r$ into a single $A d S_{5}$ space of radius $R$. (The $A d S_{5}$ is associated with the fact that the theory is dual to a conformal field theory, since its $S O(4,2)$ isometry group is also the conformal group in four spacetime dimensions.) Klebanov and Witten 
pointed out [10] that we could do the same here, as shown in Fig. 24. In flat space, a section of $\mathbf{R}^{6}$ of fixed radius was a five-sphere; here, as we discussed earlier, the corresponding section of the conifold is the space $T^{11}$, a space of $S^{2} \times S^{3}$ topology and $S U(2) \times S U(2) \times U(1)$ symmetry but with a nontrivial metric. So they proposed that the conformal field theory on the D3-branes at the conifold singularity has a stringy description as Type IIB string theory on the space $A d S_{5} \times T^{11}$. Thus the metric on the Poincare' patch (which we will work with exclusively, since our goal is eventually to study nonconformal field theory on Minkowski spacetime) is

$$
d s^{2}=R^{2} d s_{A d S_{5}}^{2}+R^{2} d s_{T^{11}}^{2}=\frac{r^{2}}{R^{2}}\left(d x^{\mu}\right)^{2}+\frac{R^{2}}{r^{2}}\left(d r^{2}+r^{2} d s_{T^{11}}^{2}\right) .
$$

Note the final expression in parentheses is the metric on the conifold, which is warped here by the $R^{2} / r^{2}$ factor. Also nonzero are the dilaton and axion, which combine together into the complex string coupling $\tau_{I I B}$ of type IIB string theory, and the self-dual 5-form, which as always in AdS/CFT tells us the number of D3-branes via Gauss's law:

$$
\left(\frac{1}{4 \pi^{2} \alpha^{\prime}}\right)^{2} \int_{T^{11}} F_{5}=N .
$$

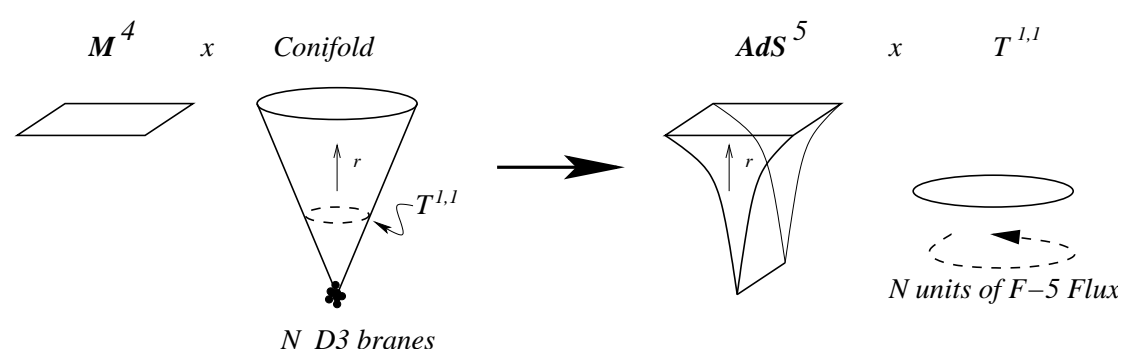

Figure 24. In the Maldacena limit, the D3-branes on Minkowski space times the conifold are replaced with an $A d S_{5} \times T^{1,1}$ space with $F_{5}$ flux on it.

Actually, this doesn't give us the full set of solutions. The string theory has two tunable parameters. One of these is the Type IIB string coupling $\tau_{I I B}$. But clearly $\tau_{I I B}$, a parameter on the space of conformal field theories, cannot be equal to the Yang-Mills gauge coupling. Instead it must correspond to an exactly marginal coupling in the gauge theory. In particular, it must be related to the $\tau_{+}$that we decided to use as a coordinate on the space of conformal fixed points, since both are invariant under $g_{1} \leftrightarrow g_{2}$. 
The other tunable parameter, which presumably is something like $\tau_{-}$, is associated with the presence of a non-trivial two-cycle in the space $T^{11}$, and a corresponding volume-form $\omega_{2}$. We do no harm to the supergravity equations if we turn on a constant complex two-form $B_{2}+i C_{2} \propto \omega_{2}$, where $B_{2}\left(C_{2}\right)$ is the Neveu-Schwarz (Ramond-Ramond) two-form of IIB string theory. (Note $H_{3}, F_{3}$ are zero since $d \omega_{2}=0$.) In fact, a little work on the string theory side - for instance, see Hanany and Uranga 1998 - shows that (when all theta angles are zero, for simplicity),

$$
\operatorname{Im}\left(\tau_{-}\right)=\operatorname{Im}\left(\tau_{1}\right)-\operatorname{Im}\left(\tau_{2}\right) \sim 2 \operatorname{Im}\left(\tau_{I I B}\right)\left[\left(\frac{1}{4 \pi^{2} \alpha^{\prime}} \int_{S^{2}} B_{2}-\frac{1}{2}\right) \bmod 1\right] .
$$

Note that $B_{2}$ is actually not zero when the gauge couplings are equal. We are naturally led to identify $\frac{1}{4 \pi^{2} \alpha^{\prime}} \int B_{2}-\frac{1}{2}$ with $\tau_{-} / 2 \tau_{+}$, which is also, via a duality, periodic with period 1. As we discussed earlier, this means that a shift of $\frac{1}{4 \pi^{2} \alpha^{\prime}} \int B_{2}$ by 1 is related to a Seiberg duality transformation on one of the two gauge groups. More precisely, this shift moves the theory from one region in the manifold of fixed points over to a second that is related to the first by Seiberg duality.

An important check on this proposal is that the supergravity agrees that operator dimensions are quantized in units of $3 / 4$ and carry appropriate $S U(2)_{L} \times S U(2)_{R} \times U(1)_{\mathcal{R}}$ quantum numbers. The gauge invariant operators of the conformal field theory should be $\operatorname{tr}\left[A_{r} B_{u}\right], \operatorname{tr}\left[A_{r} B_{u} A_{s} B_{v}\right]$, $\operatorname{tr}[A B A B A B]$, etc., (with indices in each $S U(2)$-flavor group symmetrized and with traces removed), and these should have dimension $3 / 2,3,9 / 2$, etc. Supergravity on $A d S_{5} \times T^{11}$, when reduced to five-dimensional supergravity on $A d S_{5}$, does indeed have five-dimensional scalar fields with the corresponding charges and five-dimensional masses. I won't cover this straightforward calculation here; the interested reader can read the papers by Gubser [16] and by Ceresole et al. [17]

Yet another check, due to Gubser and Klebanov [18], involves the presence of "dibaryon operators,"

$$
\mathcal{D}=\epsilon^{a_{1} a_{2} a_{3} \ldots a_{N}} \epsilon_{\alpha_{1} \alpha_{2} \alpha_{3} \ldots \alpha_{N}}\left(A_{r_{1}}\right)_{a_{1}}^{\alpha_{1}}\left(A_{r_{2}}\right)_{a_{2}}^{\alpha_{2}} \ldots\left(A_{r_{N}}\right)_{a_{N}}^{\alpha_{N}}
$$

where two epsilon tensors create gauge-invariants of the two $S U(N)$ gauge group factors. Note the $r_{i}$ are automatically symmetrized. There is a similar di-anti-baryon operator, of course. These operators have dimension $3 N / 4$ in the gauge theory. Their appearance in the gravity theory is very interesting. A D3-brane can be wrapped nontrivially on $T^{11}$, since $T^{11}$ contains (topologically) an $S^{3}$. (More precisely, $\pi_{3}\left(T^{11}\right)=\mathbf{Z}$.) From 
the perspective of $A d S_{5}$, this D-brane, with its three world-volume spatial directions wrapped on a compact subspace, looks like a particle - something created by an ordinary field in $A d S_{5}$. It therefore corresponds to a local operator in the field theory. Its topological charge corresponds to an additive charge in the gauge theory, which we are naturally led to identify with baryon number. And the object of baryon number 1 with lowest dimension is the D3-brane of lowest mass with wrapping number 1; it has mass of order $R^{4} / g_{s} \propto N$, and a complete calculation shows that indeed it corresponds to an object of dimension $3 N / 4$. Again the reader is referred to the original papers on the subject.

Gubser and Klebanov also raised another question. Since $\pi_{3}\left(T^{11}\right)=\mathbf{Z}$, we could wrap D3-branes on $T^{11}$ and get dibaryons; but $\pi_{2}\left(T^{11}\right)=\mathbf{Z}$ also. What can we use this for? They argued [18] that we could change the theory - in particular, change the gauge group from $S U(N) \times S U(N)$ to $S U(N+M) \times S U(N)$ - by wrapping $M$ D5-branes on the $S^{2}$ of $T^{11}$. Each wrapped D5-brane has two world-volume directions on the $S^{2}$, so from the $A d S_{5}$ directions it looks like a 3 -brane. There are many contexts in which $\mathrm{D} p+2$ branes wrapped on an $S^{2}$ give a fractional Dp brane - a brane with $p+1$ large world-volume dimensions but with fractional charge. This is a subject all its own, and there's no time for it here, but I refer you to the references in Gubser and Klebanov's paper. If we do wrap a D5brane of this type on an $S^{2}$, its tension grows with $r$, so it prefers to sit at $r=0$. Thus, unlike an integer D3-brane, which is free to move around on the conifold, fractional D3-branes must be placed at the singularity of the conifold, at $r=0$. This is shown in Fig. 25.

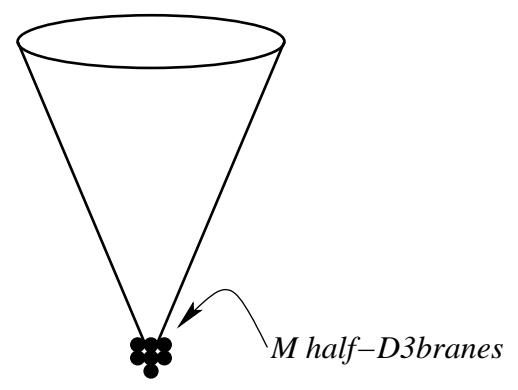

Figure 25. Fractional D3-branes must sit at the singularity, unlike full D3-branes which are free to roam anywhere on the conifold. 
The presence of D5-branes breaks the S-duality of the IIB-string theory, thereby removing the $\tau_{+} \rightarrow-1 / \tau_{+}$duality of the field theory. The D5-branes are a source for the 6 -form potential $C_{6}$, and generate a corresponding 7 -form electric flux $F_{7}$. The indices of the nonzero component of $F_{7}$ span the world-volume directions of the D5-brane and the radial direction $r$. We can Poincare-dualize $F_{7}$ to a 3 -form flux $F_{3}=* F_{7}$, which will lie perpendicular to the volume of the D5-branes, and to the coordinate $r$. In short, $F_{3}$ will be proportional to the volume form of the $S^{3}$, which we will call $\omega_{3}$, and will satisfy $\frac{1}{4 \pi^{2} \alpha^{\prime}} \int F_{3}=M$, counting the number of D5-branes.

The presence of $F_{3}$ will now act as a source for the metric, and for $F_{5}$ (and potentially for the dilaton, although we will see later that the dilaton is not in the end affected.) Moreover, $B_{2}$ will no longer be constant, and consequently $H_{3}=d B_{2}$ will be nonzero. Therefore, once the effect of the $M$ fractional branes is accounted for, the supergravity metric can no longer be $A d S_{5} \times T^{11}$. This is consistent with the gauge theory: once the two gauge groups no longer have the same number of colors, the theory is no longer conformal. But what then is the metric in the presence of fractional branes, and, if we can find the answer, what field theory physics does it correspond to?

\section{The Cascade: Preliminaries}

The answer, of course, is the duality cascade. I am not going to tell you the detective story which led to its discovery. That would take too long, and would require us to study issues which are not essential for the end result. Instead, for pedagogical reasons, we will build the cascade not from the top down, as was done historically, but from the bottom up.

\subsection{The Base of the Cascade}

What would happen if we had only fractional D3-branes and no integer D3-branes? Our field theory would then be $\mathcal{N}=1 S U(M) \times S U(0)$ gauge theory, and would have matter fields in the $(\mathbf{M}, \mathbf{0})$ representation - in short, no matter fields at all. Thus the theory would be pure $S U(M)$ $\mathcal{N}=1$ Yang-Mills, whose properties you know. Classically, it has gluons and gluinos, and a $U(1)$ R-symmetry which rotates the gluinos by $\lambda \rightarrow \lambda e^{i \alpha}$. It has no moduli space; there are no scalar fields, corresponding to the fact that the fractional branes must sit at the origin of the conifold and cannot move off of it. At one loop, it has a beta function with $b_{0}=3 M$, and a 
holomorphic strong-coupling scale satisfying

$$
\Lambda_{0}^{3 M}=\mu^{3 M} \exp \left[-8 \pi^{2} / g^{2}(\mu)\right] .
$$

It also has an anomaly which breaks the $U(1)_{\mathcal{R}}$ to a $\mathbf{Z}_{2 M}$ R-symmetry, under which the gluinos rotate as above but with $\alpha=\pi k / M, k=$ $0, \ldots, 2 M-1$. Nonperturbatively the theory has many interesting features, some analogous to pure nonsupersymmetric Yang-Mills, some analogous to QCD, and some different from both. These include

- A discrete spectrum, with a mass gap;

- Confinement (and associated strings carrying electric flux);

- Chiral symmetry breaking (a $\langle\lambda \lambda\rangle$ condensate spontaneously breaks $\mathbf{Z}_{2 M}$ to $\mathbf{Z}_{2}$, since now the vacuum is invariant only under chiral transformations with $\alpha=0, \pi)$; and its two consequences:

- $M$ identical isolated degenerate vacua, with the gluino condensate taking values $\langle\lambda \lambda\rangle=e^{2 \pi i n / M}\left(\Lambda_{0}^{3 M}\right)^{1 / M}, n=0, \ldots, M-1$ (since as with any spontaneously broken symmetry $G \rightarrow H$, the vacua must form a representation of $G / H \approx \mathbf{Z}_{2 M} / \mathbf{Z}_{2} \approx \mathbf{Z}_{M}$ ); and

- Domain walls which separate one vacuum from the next - and it turns out these are BPS saturated (Dvali and Shifman, 1997).

But this is very interesting already. We know that the conifold has a continuous $U(1)_{\mathcal{R}}$ symmetry. If we put $M$ fractional branes on it, it must somehow happen that (a) the continuous symmetry is broken by an anomaly to $\mathbf{Z}_{2 M}$, and (b) the remaining symmetry is spontaneously broken to $\mathbf{Z}_{2}$ by the dynamics of the theory. How is this going to play out? We won't discuss the anomaly here (see Ouyang, Klebanov and Witten [19]) but we will carefully investigate the spontaneous breaking of chiral symmetry.

\subsection{The Base with an Extra D3}

What if we had $M$ fractional branes and one integer D3-brane, as in Fig. 26? This is a particularly interesting case. The D3-brane is free to move all around the conifold, unlike the fractional branes. As such, it makes an excellent probe of the space, and what has happened to it. We will see in a moment that it serves our purpose admirably.

The field theory on the D3-branes is $S U(M+1) \times S U(1)$ with fields $A_{r}$ in the $(\mathbf{M}+\mathbf{1}, \mathbf{1})$ and $B_{u}$ in the $(\overline{\mathbf{M}+\mathbf{1}}, \mathbf{1})$ representations. But $S U(1)$ is no group at all, so this is simply SQCD with $M+1$ colors and 2 flavors. However, it isn't quite that trivial, because the flavors 


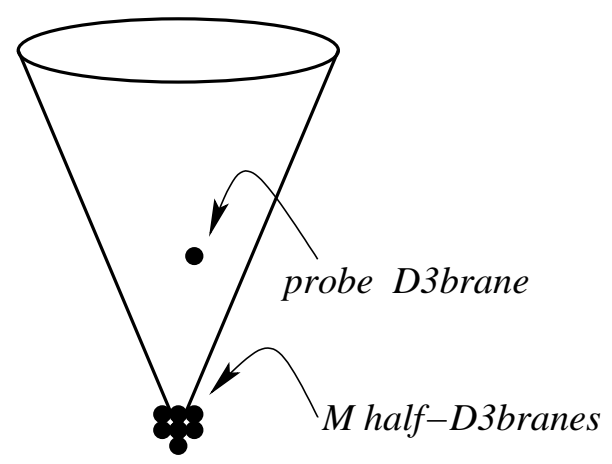

Figure 26. $M$ fractional D3-branes stuck at the origin of the conifold, with one full D3-brane serving as a probe.

will have the conifold superpotential, which classically preserves the full $S U(2)_{L} \times S U(2)_{R} \times U(1)_{\mathcal{R}} \times U(1)_{B}$ flavor symmetry, namely

$$
W=h\left[\left(A_{1} B_{1}\right)\left(A_{2} B_{2}\right)-\left(A_{1} B_{2}\right)\left(A_{2} B_{1}\right)\right]=h \operatorname{det}_{r, u}\left(A_{r} B_{u}\right) .
$$

Classically, our single D3-brane knows that it is moving on the conifold. The moduli space must be described by the four gauge invariant operators $Z_{11}=A_{1} B_{1}, Z_{22}=A_{1} B_{2}, Z_{21}=A_{2} B_{1}, Z_{22}=A_{2} B_{2}$. But, as we have seen before, the classical equations for a supersymmetric vacuum include

$$
0=\frac{\partial W}{\partial A_{1}}=B_{1}\left(A_{2} B_{2}\right)-B_{2}\left(A_{2} B_{1}\right)
$$

which gives (multiplying by $A_{1}$ and contracting the hanging $S U(M+1)$ indices)

$$
0=\left(A_{1} B_{1}\right)\left(A_{2} B_{2}\right)-\left(A_{1} B_{2}\right)\left(A_{2} B_{1}\right)=\operatorname{det} Z_{r u}
$$

which is exactly the equation for the conifold.

At one loop, an anomaly removes most of the $U(1)_{\mathcal{R}}$ symmetry. However, there is a discrete R-symmetry which survives. The superpotential, which must have R-charge 2 , requires the $A$ s and $B$ s have $\mathrm{R}$-charge $\frac{1}{2}$, and their fermions carry charge $-1 / 2$. The gluinos carry R-charge 1 . An instanton in this theory has one fermion zero mode for each of $A_{1}, A_{2}, B_{1}, B_{2}$, and $2(M+1)$ for the gluinos. If the gluinos are rotated by a phase $e^{i \alpha}$, then the $A, B$ zero modes rotate by a phase $e^{-i \alpha / 2}$. Thus the whole instanton rotates by a phase $e^{i(2(M+1) \alpha-2 \alpha)}=e^{i 2 M \alpha}$. For the instanton to be invariant (meaning that the R-charge in question is anomaly-free,) it must be that 
$(2 M) \alpha$ is a multiple of $2 \pi$, or $\alpha=\pi k / M, k=0, \ldots, 2 M-1 .{ }^{17}$ This $\mathbf{Z}_{2 M}$ non-anomalous discrete R-symmetry is a chiral symmetry, since it rotates $A$ and $B$ in the same direction. Remarkably, this calculation suggests that our theory has a discrete R-symmetry which only depends on the number of fractional D3-branes, and not on the number of integer D3-branes.

What does this theory do nonperturbatively? When $h \rightarrow 0$ this is just SQCD again, and we know that for $N_{f}=2$ the theory is best described using the gauge-invariant variables $Z_{r u}=A_{r} B_{u}$ (as long as all fields have small expectation values.) And we know (from Affleck, Dine and Seiberg [20], as described in Intriligator's lectures) that the theory generates a dynamical superpotential of the form

$$
W=(M-1)\left[\frac{2 \Lambda^{3(M+1)-2}}{\operatorname{det} Z}\right]^{1 /([M+1]-2)} .
$$

where $\Lambda$ is the holomorphic strong coupling scale of the $S U(M+1)$ gauge group. The condition for a supersymmetric vacuum becomes

$$
0=\frac{\partial W}{\partial Z_{u}^{r}}=-\left(2 \Lambda^{3 M+1}\right)^{1 /(M-1)}(\operatorname{det} Z)^{-M /(M-1)} \epsilon_{r s} \epsilon^{u v} Z_{v}^{s}
$$

and so this theory has no vacuum except at $\operatorname{det} Z \rightarrow \infty$. On the other hand, when $h$ is present but small, the effective superpotential is of the form

$$
W=(M-1)\left[\frac{2 \Lambda^{3 M+1}}{\operatorname{det} Z}\right]^{1 /(M-1)}+h \operatorname{det} Z+\operatorname{order}\left(h^{2}\right) .
$$

Let us assume that the higher order terms in $h$ vanish (and one can show, using nonperturbative methods, that they do in this case.) Then the conditions for a supersymmetric vacuum take the form

$$
0=\frac{\partial W}{\partial Z_{u}^{r}}=\left[-\left(2 \Lambda^{3 M+1}\right)^{1 /(M-1)}(\operatorname{det} Z)^{-M /(M-1)}+h\right] \epsilon_{r s} \epsilon^{u v} Z_{v}^{s}
$$

Despite appearances, there is no solution at $Z_{v}^{s}=0$; if you take the determinant of (3.5), you will see this is the case. The only solutions are to have the quantity in brackets vanish:

$$
(\operatorname{det} Z)^{M}=\left[2 \Lambda^{3 M+1} / h^{M-1}\right] \Rightarrow \operatorname{det} Z=\left[2 \Lambda^{3 M+1} / h^{M-1}\right]^{1 / M} .
$$

\footnotetext{
${ }^{17}$ We only include $k$ up to $2 M-1$ because the apparently additional symmetry under which $\alpha=2 \pi$, the gluinos are invariant, and $A$ and $B$ change sign is already contained in $U(1)_{B}$ as a rotation by $\pi$; thus it is already included in our list of symmetries.
} 
This is a remarkable result, in a wide variety of ways. First, we see that the probe D3-brane is not moving on the conifold anymore! Instead, it moves on a space with a different complex structure, given by the equation $\operatorname{det} \tilde{z}_{r u}=\epsilon$, where $\epsilon$ is a nonzero complex constant. This space is also well-known and well-studied [12]: it is called the "deformed conifold." 18

Moreover, since Eq. (3.6) has $M$ solutions for $\operatorname{det} Z$, the constant $\epsilon$ can have any one of $M$ different phases, so the moduli space consists not of one copy of this space but of $M$ copies. This is reminiscent of the fact that there are $M$ different vacua in $S U(M)$ Yang-Mills theory. Indeed, we might guess that what has happened is that (in analogy to $S U(M)$ YangMills) the expectation values for $Z_{r u}$ have broken the $\mathbf{Z}_{2 M}$ symmetry down to $\mathbf{Z}_{2}$. This guess is correct! Let's ignore the anomaly for the moment, and see that the classical $U(1)_{\mathcal{R}}$ continuous symmetry is broken down to $\mathbf{Z}_{2}$. When $\epsilon=0$ we can rotate the $\tilde{z}_{r u}$ by any phase without changing the equation $\operatorname{det} \tilde{z}=0$, but when $\epsilon \neq 0$ only $\tilde{z}_{r u} \rightarrow-\tilde{z}_{r u}$ for all $r, u$ leaves $\operatorname{det} \tilde{z}=\epsilon$ invariant. The anomaly does not affect this remaining $\mathbf{Z}_{2}$ symmetry. Thus the $U(1)_{\mathcal{R}}$ symmetry behaves just as in pure Yang-Mills; the classical $U(1)$ is broken explicitly by the anomaly to $\mathbf{Z}_{2 M}$, and then it is broken spontaneously to $\mathbf{Z}_{2}$, with the result that the moduli space of the theory consists of $M$ identical branches, with domain walls separating them.

However, unlike $\mathcal{N}=1$ Yang-Mills, this theory has massless scalar fields, six to be precise, corresponding to the six coordinates of the probe D3brane. And the chiral $S U(2)_{L} \times S U(2)_{R}$ symmetry, which is not present in the pure Yang-Mills case, is also broken - to the diagonal $S U(2)_{L+R}$ subgroup if $Z_{11}=Z_{22}=\sqrt{\operatorname{det} Z}$ and $Z_{12}=Z_{21}=0$ (or any flavor rotation of this vacuum), and further to $U(1)_{L+R}$ otherwise. Thus the theory has Nambu-Goldstone bosons, one for each broken generator of the group, along with other scalar and fermion superpartners. Note these three (or five) "pions" are simply a subset of the six coordinates of the probe brane.

Also remarkable is that the moduli space is now nonsingular. Recall that any section of the conifold at a fixed radial coordinate $r$ is of the form $T^{11}$, which has topology $S^{2} \times S^{3}$, and that the radius of these spheres goes to zero in the limit $r \rightarrow 0$, leading to a singularity, as shown in Fig. 21. This is not true of the deformed conifold. Again let $z_{i}=x_{i}+i y_{i}$; and take $\epsilon$ to be real and positive. (If it is not, then redefine $z_{i}=e^{i(\arg \epsilon) / 2}(x+i y)$,

\footnotetext{
${ }^{18}$ The "resolved conifold" is yet another space, with the same complex structure as the
} conifold but with other aspects changed; I will not discuss it here. 


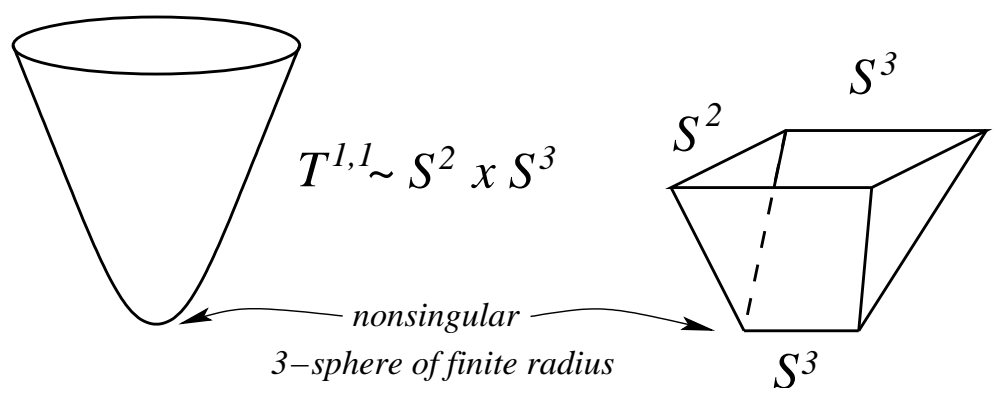

Figure 27. The conifold is deformed by the $M$ fractional D3-branes; the singularity is gone, because the $S^{3}$ remains of finite size as the radius of the $S^{2}$ shrinks to zero. Compare with Fig. 21.

and proceed in the same way.) Then the deformed conifold is defined by

$$
\sum_{i} x_{i} y_{i}=0 ; \sum x_{i}^{2}=r^{2} / 2+\epsilon / 2 ; \sum y_{i}^{2}=r^{2} / 2-\epsilon / 2 .
$$

Since we chose $\epsilon>0$, and $r^{2}>0$, we can always take $x_{i}$ to be a fourdimensional real vector of length $\sqrt{r^{2}+\epsilon} / \sqrt{2}$, which parametrizes a 3 sphere. Now we must take $y_{i}$ to be a four-dimensional real vector, lying orthogonal to $x_{i}$, of length $\sqrt{r^{2}-\epsilon} / \sqrt{2}$. This gives us a 2-sphere if $r^{2}>\epsilon$, but for $r=\sqrt{\epsilon}$ we see this 2-sphere degenerates to a point. Note there is still a nontrivial 3 -sphere at this radius. Clearly there are no solutions for $r<\sqrt{\epsilon}$. Thus our space has the property that the 2 -sphere shrinks faster than the 3 -sphere, and the space ends entirely when the 2 -sphere disappears. Locally, near $r=\sqrt{\epsilon}$, the 6-dimensional space looks topologically like $\mathbf{R}^{3}$ fibered over a finite-radius $S^{3}$, which is a nonsingular fibration. This is illustrated in Fig. 27, to be compared with Fig. 21.

Now, what is the physics that has caused this to happen? Does it have a simple description? If $Z_{11} \sim Z_{22} \sim \sqrt{\operatorname{det} Z} \leq \Lambda$, then it doesn't; the theory is more or less perturbative down to the scale of order $\Lambda$, and below that scale the physics is strongly coupled. However, we can study the theory in a different regime, where it is more tractable. With a fixed $\operatorname{det} Z$, we can take, for instance, $Z_{11}$ large, while sending $Z_{22}$ very small. In this limit, the gauge group is broken at high energy, by one unit, to $S U(M)$. The superpotential, together with the expectation value for $A_{1}$ and $B_{1}$, gives $A_{2}$ and $B_{2}$ a mass $h\left\langle A_{1} B_{1}\right\rangle$. Below this scale the theory is a pure $S U(M) \mathcal{N}=1$ Yang-Mills theory, with a scale $\Lambda_{0}$ satisfying $\Lambda_{0}^{3 M}=h \Lambda^{3 M+1}$. (Note this scale, and 
much of the ensuing low-energy physics, is independent of $Z_{11} / Z_{22}$. This is a common feature of the holomorphic subsector of supersymmetric theories; see Argyres et al. [8]) And so gluino condensation occurs, generating the low-energy superpotential, confinement, breaking of the $\mathbf{Z}_{2 M}$ symmetry, and all of the other details we expect. The only thing new is that we can do $S U(2)_{L} \times S U(2)_{R}$ rotations on each of these vacua, generating a larger moduli space for each choice of gluino condensate; this is, in the end, the only effect of the probe brane on the $M$ fractional branes.

The implications for supergravity are clear. The fractional branes must somehow turn the conifold into the deformed conifold. The probe brane will have little effect on the whole structure (which justifies thinking of it as a probe) and in supergravity it is likely to play almost no role (except for the nontrivial fact that the matter it adds can break the confining flux tubes of the theory.)

\subsection{More Integer D-branes: $S U(N+M) \times S U(N)$}

We could also consider the effect of two D3-branes. This is covered partially in the appendix of Klebanov and Strassler [21], and we do not need it here; the results are similar to the case just discussed. More interesting is when the number of D3-branes is of order $M$, or even greater.

Let us see what we can say classically and semiclassically about the theory with $N$ D3-branes and $M$ fractional D3-branes. The gauge group is $S U(N+M) \times S U(N)$, and the matter and superpotential are as usual for the conifold. The $S U(2) \times S U(2)$ and baryon symmetries are as usual. Again the $U(1)_{\mathcal{R}}$ is broken by anomalies. We must assign $R_{A}=R_{B}=\frac{1}{2}$ to be consistent with the superpotential (although this does not imply $\operatorname{dim} A=$ $\operatorname{dim} B=\frac{3}{4}$, since we are not necessarily going to reach a conformal fixed point!) Let us consider an instanton in the $S U(N+M)$ gauge fields. Such an instanton has $2(N+M)$ gluino zero modes. Meanwhile, a fermion in the fundamental representation of $S U(N+M)$ provides one zero mode; there are $2 N$ such fermions, $N$ each from $A_{1}$ and $A_{2}$. Similarly, there are $2 N$ zero modes from $B_{1}$ and $B_{2}$. The $A$ and $B$ fermions have R-charge $-1 / 2$, and the gluinos have R-charge 1 , so under a $U(1)_{\mathcal{R}}$ rotation by a phase $e^{i \alpha}$ the instanton rotates by a phase $\exp \{i[2(N+M)-2 N] \alpha\}=e^{i(2 M) \alpha}$. To avoid an anomaly, then, we must have $\alpha=\pi k / M, k=0,1 \ldots, 2 M-1-$ again a $\mathbf{Z}_{2 M}$ symmetry.

What happens if we apply this $\mathbf{Z}_{2 M}$ symmetry to an instanton of the $S U(N)$ gauge group? We simply switch $N$ and $N+M$ in the above calcu- 
lation, and find the instanton rotates by $e^{-i(2 M) \alpha}=1$. So the same $\mathbf{Z}_{2 M}$ symmetry is anomaly-free under both groups. A remarkable result - the anomaly-free discrete R-symmetry of the $S U(N+M) \times S U(N)$ theory is a $\mathbf{Z}_{2 M}$, independent of $N$ !

Now, what about the RG flow of the theory? The one-loop beta functions for the theory are given by $b_{0}^{(N+M)}=3(N+M)-2 N=N+3 M$ and $b_{0}^{(N)}=3 N-2(N+M)=N-2 M$; thus it appears both gauge groups are asymptotically free, but the $S U(N+M)$ group, which has less matter, flows faster. This conclusion is partially correct, but the reasoning is basically wrong. We need to be more careful.

\subsection{The Lowest Step of the Cascade}

Let's study in detail the case of $N=M$, that is, $S U(2 M) \times S U(M)$, with $A_{r}$ in the $(\mathbf{2 M}, \overline{\mathbf{M}})$ representation and $B_{u}$ in the conjugate representation. The superpotential is the usual one, Eq. (2.1). In this case $b_{0}^{(2 M)}=4 M$ while $b_{0}^{(M)}=-M$, so the second group is (even naively) infrared free. The lowenergy dynamics is therefore dominated by $S U(2 M)$, with $S U(M)$ acting as an unimportant "spectator." The $S U(2 M)$ group has $2 M$ flavors $(M$ from $A_{1}$ and $B_{1}$, and $M$ from $A_{2}$ and $B_{2}$ ). Such a theory is an example of SQCD with an equal number of flavors and colors. It is well-described by its mesons $A_{r} B_{u}$ and by its baryons $\mathcal{B}=[A]^{2 M}$ and $\overline{\mathcal{B}}=[B]^{2 M}$, which we recognize as related to (but not equal to) the dibaryons of $S U(N) \times S U(N)$ discussed earlier. But we must take our time here. The mesons $\left(Z_{r u}\right)_{b}^{a}=$ $\left(A_{r}\right)_{\alpha}^{a}\left(B_{u}\right)_{b}^{\alpha}$ (which are in the $(\mathbf{2}, \mathbf{2})$ representation of $\left.S U(2)_{L} \times S U(2)_{R}\right)$ have their $S U(2 M)$ indices contracted, but not their $S U(M)$ indices. To form irreducible representations of $S U(M)$, we must define

$$
Z_{r u}^{0}=\left(Z_{r u}\right)_{a}^{a},\left(Z_{r u}^{a d j}\right)_{b}^{a}=\left(Z_{r u}\right)_{b}^{a}-\frac{1}{M} Z_{r u}^{0} \delta_{b}^{a}
$$

which are in the singlet and in the adjoint representation, respectively, of $S U(M)$. Meanwhile, the baryon $\mathcal{B}$, an $S U(2 M)$ gauge invariant operator, is also (by Bose-statistics) both an $S U(M)$ and an $S U(2)_{L}$ singlet. The same is true for $\overline{\mathcal{B}}$.

SQCD with $2 M$ flavors and $2 M$ colors has a "deformed moduli space," as shown by Seiberg [22]. This can be implemented using a Lagrange multiplier superfield $X$ in the effective superpotential, which takes the form

$$
W=h \operatorname{tr}_{a, b}\left[Z_{11} Z_{22}-Z_{12} Z_{21}\right]-X\left(\underset{r, u, a, b}{\operatorname{det}} Z-\mathcal{B} \overline{\mathcal{B}}-\Lambda_{2 M}^{4 M}\right)
$$


Notice the determinant over $r, u, a, b$ treats $Z$ as a $2 M \times 2 M$ matrix, not as a $2 \times 2$ matrix. There are multiple solutions to this equation, and I don't want to go exploring them here. But the simplest solution is

$$
\mathcal{B}=\overline{\mathcal{B}}=i \Lambda_{2 M}^{2 M}, Z^{a d j}=0, Z_{r u}^{0}=0 .
$$

Since $\mathcal{B}, \overline{\mathcal{B}}$ are singlets of $S U(M)$, they leave the $S U(M)$ gauge group unbroken. Meanwhile, the $Z$ fields are massive. The theory at energies below their masses consists simply of an unbroken $S U(M)$ gauge group with no massless matter: a pure $\mathcal{N}=1$ Yang-Mills theory, as illustrated in Fig. 28. Again we have confinement, chiral symmetry breaking, and all of the other phenomena we have discussed up to now.

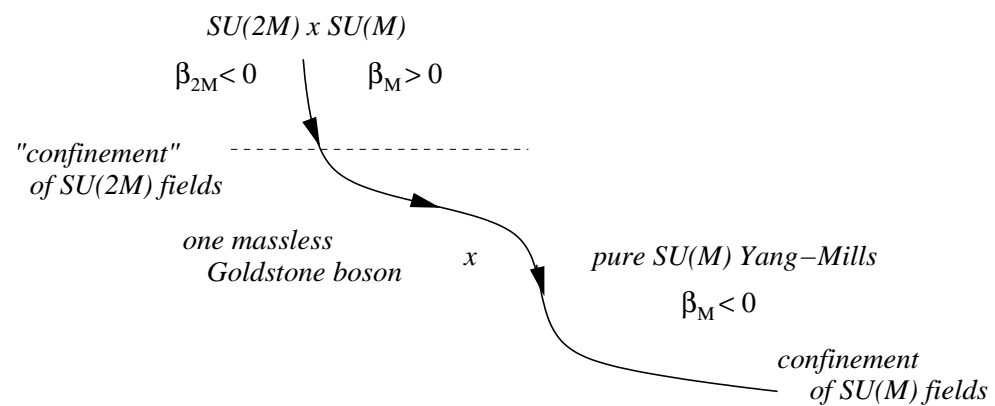

Figure 28. At one scale, $S U(2 M)$ confines, leaving behind a pure $S U(M) \mathcal{N}=$ 1 Yang-Mills theory plus a single massless goldstone supermultiplet (from the spontaneously broken baryon number). The goldstone supermultiplet couples only through irrelevant operators to the $S U(M)$ sector, and does not affect its infrared dynamics.

This is almost true. The symmetry $U(1)_{B}$ is broken by the expectation values for $\mathcal{B}$ and $\overline{\mathcal{B}}$. (Notice that no other symmetries are broken; the $\mathbf{Z}_{2 M}$ R-symmetry and the $S U(2)_{L} \times S U(2)_{R}$ global symmetries are retained.) Consequently, there must also be a massless Goldstone boson which rotates the phases of $\mathcal{B}$ and $\overline{\mathcal{B}}$ in opposite directions. (Here it is crucial that $U(1)_{B}$ is not gauged in the quantum theory; the subtle issues involved were partly explained by Aharony [23] and finally clarified by Gubser, Herzog and Klebanov [24].) Thus there isn't actually a mass gap in this theory, though there is still a discrete spectrum. The Goldstone mode, its scalar superpartner (which changes the ratio $|\mathcal{B} / \overline{\mathcal{B}}|$ ) and a partner fermion form a massless chiral multiplet. It is important to note, however, that the extra 
massless chiral multiplet has very minor effects upon the interesting dynamics of the confining $S U(M)$ gauge theory. Because it is a composite of the $S U(2 M)$ dynamics, contains $2 M \gg 1$ constituents, is neutral under $S U(M)$, and couples to the $S U(M)$ sector only via irrelevant operators, it does not participate in the $S U(M)$ dynamics. Even with this massless multiplet present, the $S U(M)$ sector confines in the usual way, and generates a mass gap as usual in its own sector.

In summary, when the $S U(2 M)$ sector confines, (1) it makes neutral scalar baryons, whose expectation values break baryon number and give rise to a single massless composite chiral multiplet, and also (2) it makes scalar mesons charged under $S U(M)$, but these are massive because of the superpotential, leaving a pure Yang-Mills theory in the $S U(M)$ sector. At the scale of the confining $S U(M)$ dynamics, and at small 't Hooft coupling, the $S U(M)$ sector and the sector containing the massless chiral multiplet are exponentially-weakly coupled. This is because all couplings of the Goldstone mode are suppressed by $\Lambda_{2 M}$, which is exponentially larger than $\Lambda_{M}$. At strong 't Hooft coupling, the situation is more subtle, and we will return to it only once our understanding of the cascade is complete. Still, we will see that even then the presence of the Goldstone supermultiplet has limited effects on the dynamics, at least when $|\mathcal{B}|=|\overline{\mathcal{B}}|$.

\subsection{Another Step}

Let's try one more example before climbing higher on the cascade. What if $N=2 M$ ? Then we have $S U(3 M) \times S U(2 M)$ to start with. The $S U(2 M)$ theory has $b_{0}=0$ and is again infrared-free. Let's initially ignore it, as we did for $N=M$ (this will be justified more fully later.) Then the $S U(3 M)$ theory becomes strongly coupled at some scale $\Lambda_{3 M}$, where its coupling $g_{3}$ becomes large, and we must ask what it does there. In this regime the theory is effectively SQCD with $3 M$ colors, with $2(2 M)=4 M$ flavors, plus the quartic superpotential $W=h \operatorname{tr} \operatorname{det}\left[A_{r} B_{u}\right]$. This theory is in the free-magnetic phase: below $\Lambda_{3 M}$ (momentarily ignoring the spectator $S U(2 M)$ group and the small superpotential) the $S U(3 M)$ sector is better described as $S U(M) \mathrm{SQCD}+\mathrm{M}$ with $4 M$ flavors $a^{u}, b^{r}$, which is infrared free, along with $S U(M)$-gauge-singlet operators $\left(Y_{r u}\right)_{\beta}^{\alpha}=A_{u}^{\alpha} B_{r \beta}$, where $\alpha, \beta$ are indices in the $S U(2 M)$ spectator group. The superpotential is

$$
W=\hat{h} \operatorname{tr}_{\alpha} \operatorname{det}_{r, u} Y+y Y_{r u} a^{r} b^{u}
$$


$(S U(2 M)$ and $S U(M)$ indices contracted implicitly.) Integrating out $Y$ we obtain

$$
W=\tilde{h} \operatorname{tr} \operatorname{det}_{r, u}\left(a^{r} b^{u}\right)
$$

where $\tilde{h} \sim-y^{2} / 4 \hat{h}$. Indeed this is just the sort of duality transformation with quartic operators that we studied in Sec. 1.4; see Eqs. (1.16)-(1.17).

Exercise: Verify the previous paragraph! You'll need to fully grasp it in order to understand the self-similarity of the duality cascade.

Now we recall that the coupling of the $S U(2 M)$ group is not quite zero, even if it is small. Below $\Lambda_{3 M}$, where we usefully change variables from an $S U(3 M)$ description to an $S U(M)$ description, we now have an $S U(2 M) \times S U(M)$ theory of exactly the form that we discussed in Sec. 3.4: one with $M$, not $2 M$, integer D3-branes (in addition to $M$ fractional branes) on the conifold. We already know what happens in this theory. The $S U(2 M)$ group now has a negative beta function $\left(b_{0}=+4 M\right)$. Although it remained weakly-coupled above the $S U(3 M)$ strong-coupling transition, it now flows to strong coupling. When its coupling $g_{2}$ becomes large, we again change variables to a hadronic description using the mesons and baryons constructed from $S U(2 M)$ gauge-invariants. This leaves only a massless Goldstone mode and an $S U(M)$ gauge group with pure $\mathcal{N}=1$ Yang-Mills, which confines, breaks chiral symmetries, etc., etc.

Thus, as in Fig. 29, we now have three separate strong-coupling transitions chained together, the first of which is best described by making a Seiberg duality transformation (in fact all three transitions can be viewed this way) and the first hints of what a duality cascade is going to be. What do these dualities imply? The three theories that we have discussed, with $N=2 M, N=M$, and $N=0$ are generally different theories, whose extreme infrared physics is the same. But to say only this underestimates Seiberg's duality. As can be seen from Fig. 29, the physics of the $N=2 M$ and $N=M$ theories become equal far above the extreme infrared. Below the scale where their two flows merge, exact Seiberg duality is in operation. The $N=2 M$ and $N=M$ theories give two equivalent descriptions of the RG flow all the way from the confining scale of $S U(2 M)$ down to zero momentum. We will see that this generalizes to larger $N$. 


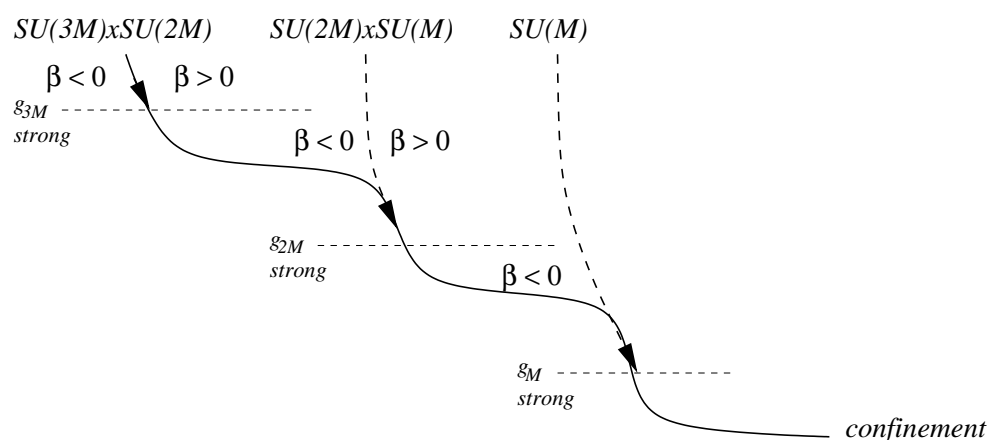

Figure 29. Three strong-coupling transitions; the bottom steps of the duality cascade.

\section{The Cascade: Descending the Grand Staircase}

Finally, it is time to move beyond individual cases to study the full duality cascade. We will first investigate the very high steps in the cascade, then understand their supergravity dual description. Next we will make some observations concerning the supergravity dual of the bottom steps. We will conclude these lectures by considering applications to QCD and to beyondthe-standard-model physics.

\subsection{The Upper Reaches of the Cascade}

Having investigated $k=1,2$ and 3 , our next task should be to study $(k-1) M$ integer D3-branes on the conifold, with $k \gg 1$ an integer. The supergravity description was gradually elucidated in papers of Klebanov with Nekrasov [26], and then with Tseytlin [25], and finally with yours truly [21], where we also understood the field theory. We will investigate the $S U(k M) \times S U([k-1] M)$ theory by using methods similar to those above, but paying special attention to an expansion in $1 / k$. The reason for this should be clear: as $k \rightarrow \infty$, the theory comes closer and closer to the Klebanov-Witten model, and our understanding of the latter should prove conceptually and technically useful.

Naively speaking, the $S U(k M)$ and $S U([k-1] M)$ theories, which have $b_{0}=3 k M-2[k-1] M=(k+2) M$ and $3[k-1] M-2 k M=(k-3) M$ respectively, are both asymptotically free, although the $S U(k M)$ theory is running faster toward strong coupling. If the $S U(k M)$ group should be replaced by its Seiberg-dual description at some strong-coupling transition, 
then since $2[k-1] M-k M=[k-2] M$, we will find ourselves (through the same arguments as above) with an $S U([k-1] M) \times S U([k-2] M)$ gauge theory of exactly the same form. Again both gauge groups are (naively) asymptotically free; but the $S U([k-1] M)$ beta function has increased $\left(b_{0}=(k+1) M\right.$ now $)$ so it now flows faster to stronger coupling, and perhaps we should dualize it, obtaining $S U([k-2] M) \times S U([k-3] M)$, again of the same form. And so we can imagine cascading downward through $k$ separate strong-coupling transitions, at each of which it is wise and convenient to replace the more-strongly-coupled gauge group with its dual description, and after each of which the theory looks the same as before except with $M$ fewer colors in both groups. This continues until we reach pure $S U(M)$ Yang-Mills theory, whereupon we get confinement, chiral symmetry breaking, etc. This is illustrated in Fig. 30.

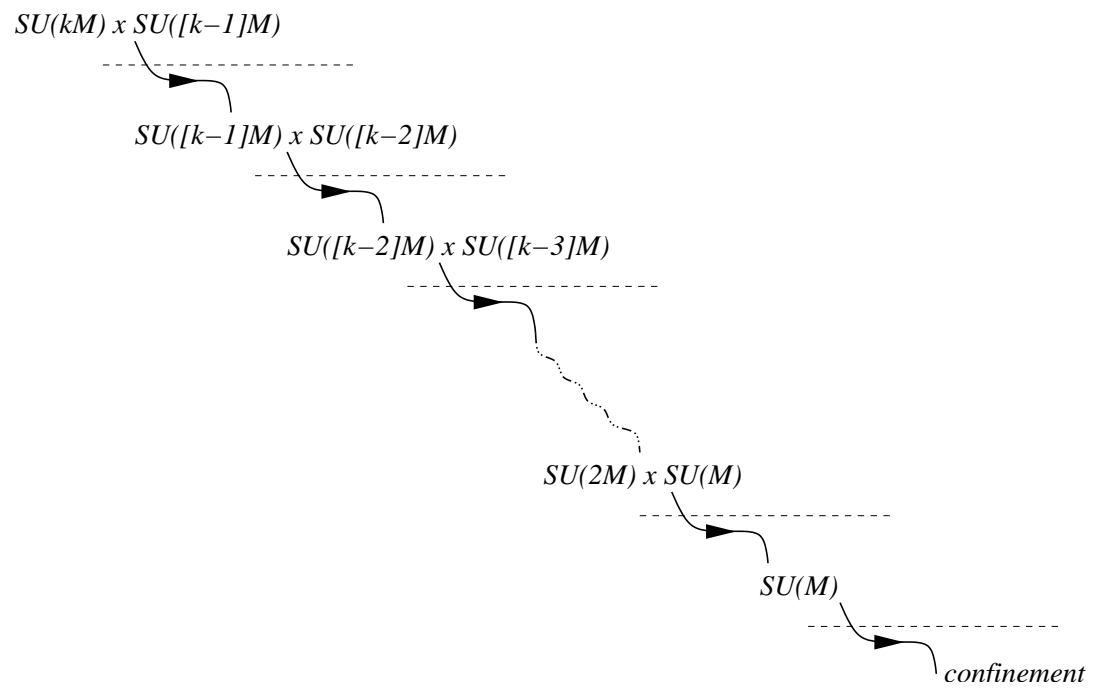

Figure 30. The duality cascade; a naive view, to be corrected later.

But while this is correct in its broadest outlines, almost all of the real physics is wrong. Our goal here is to say precisely what is happening, and in doing so bring together all of the field-theory techniques that we have gradually been assembling.

To build up an understanding of a field theory of this type takes some 
time, and we'll start slow. Let's first imagine that we have, in the far ultraviolet, a weakly-coupled theory of $S U(k M) \times S U([k-1] M)$, with gauge couplings $g_{k}, g_{k-1}$, the usual $A$ and $B$ fields, and the conifold superpotential, with coupling $h$ and dimensionless coupling $\eta=h \mu$. Since all fields have the same anomalous dimension $\gamma_{0}$, we have

$$
\begin{aligned}
\beta_{g_{k}}= & -F\left(g_{k}\right)\left[(k+2) M+2[k-1] M \gamma_{0}\right] \\
& \Rightarrow \beta_{\lambda_{k}}=-G\left(\lambda_{k}\right)\left[\left(1+\frac{2}{k}\right)+2\left(1-\frac{1}{k}\right) \gamma_{0}\right] \\
\beta_{g_{k-1}}= & -F\left(g_{k-1}\right)\left[(k-3) M+2 k M \gamma_{0}\right] \\
& \Rightarrow \beta_{\lambda_{k-1}}=-G\left(\lambda_{k-1}\right)\left[\left(1-\frac{2}{k-1}\right)+2\left(1+\frac{1}{k-1}\right) \gamma_{0}\right], \\
\beta_{\eta}= & \eta\left(1+2 \gamma_{0}\right),
\end{aligned}
$$

where

$$
F\left(g_{p}\right)=\frac{g_{p}^{3}}{16 \pi^{2}} \frac{1}{1-\left[g_{p}^{2}(p M) / 8 \pi^{2}\right]}, G\left(\lambda_{p}\right)=\frac{\lambda_{p}^{2}}{8 \pi^{2}} \frac{1}{1-\left[\lambda_{p} / 8 \pi^{2}\right]} .
$$

and $\lambda_{p}=g_{p}^{2} p M$. When the couplings are small and $\left|\gamma_{0}\right| \ll 1$, we see that both gauge couplings are asymptotically free, while $\beta_{\eta}>0$. However, the signs of the beta functions are all reversed if $\gamma_{0} \ll-1 / 2$. This is clear from the fact that, at large $k$, all three beta functions become proportional to $1+2 \gamma_{0}$. In fact, comparison with Eq. (2.3) shows that at large $k$ the beta functions of this theory become equal, up to $1 / k$ corrections, to those of the Klebanov-Witten model.

Let us recall the key features of the Klebanov-Witten theory: (1) it has a continuous two-complex-dimensional manifold of conformal fixed points, shown in Fig. 18, defined by the condition $\gamma_{0}=-1 / 2$ which causes all three beta functions (2.3) of the Klebanov-Witten model to simultaneously vanish; (2) the manifold is infrared-stable, in the sense that all renormalization group flows end, in the infrared, on the manifold, in analogy to a ball rolling with friction into a flat valley bottom; and (3) duality symmetries act on this manifold, including a blend of electric-magnetic dualities and Seiberg dualities.

However, the $1 / k$ differences between (4.1) and (2.3) are critically important. It is easily seen in (4.1) that for finite $k$ there is no value of $\gamma_{0}$ for which even two, much less three, of the beta functions simultaneously vanish. Thus, unlike the Klebanov-Witten model, this theory does 
not have continuous manifolds of conformal fixed points. Still, the theory with fractional D-branes inherits modified versions of each of the three key features of the Klebanov-Witten theory: (1) it has a region, a thin "slab" inside the three-dimensional space of couplings, defined by the condition $\gamma_{0} \approx-1 / 2$, where all three beta functions (4.1) are very small; (2) this slab is infrared-stable, in the same sense as in the Klebanov-Witten model, in that all renormalization group flows reach this slab in the infrared; and (3) although $S L(2, \mathbf{Z})$ duality is lost (since fractional D3-branes are not invariant under $S L(2, \mathbf{Z})$ transformations), Seiberg duality continues to act on this slab, as we will soon see.

Since (4.1) and (2.3) are equal as $k \rightarrow \infty$, it is not surprising that, for large $k$ and $\gamma_{0} \approx-\frac{1}{2}$, the beta functions are all of order $1 / k$ compared to their "natural size." To be more precise, let us write $\gamma_{0}=-\frac{1}{2}+\delta_{0}$; then

$$
\begin{gathered}
\beta_{g_{k}}=-F\left(g_{k}\right) M\left[3+2(k-1) \delta_{0}\right], \\
\beta_{g_{k-1}}=-F\left(g_{k-1}\right) M\left[-3+2 k \delta_{0}\right], \\
\beta_{\eta}=2 \eta \delta_{0} .
\end{gathered}
$$

For $\left|\delta_{0}\right| \sim \frac{1}{2}$, as will be the case for a generic point in the space of the coupling constants $\left(g_{1}, g_{2}, \eta\right)$, the beta functions for the gauge couplings are of order $g^{3} k M$, while $\beta_{\eta} \sim \eta$. The resulting flow, as in the KlebanovWitten model, pushes the theory toward the region where $\delta_{0}\left(g_{1}, g_{2}, \eta\right)$ is small. Once within the narrow slab of the coupling-constant space where $\left|\delta_{0}\left(g_{1}, g_{2}, \eta\right)\right| \sim 1 / k$, the couplings all slow to a crawl: the two gauge couplings have beta functions of order $g^{3} M$, and $\beta_{\eta} \sim \eta / k$, each a factor of $1 / k$ smaller in magnitude than in the rest of the coupling space. Thus the region $\left|\delta_{0}\right| \sim 1 / k$ is an infrared-stable slow-motion slab; once the couplings reach it, they remain within it, their values drifting slowly. Clearly, by the fact that this theory matches the Klebanov-Witten theory in the large- $k$ limit, the location of this slab is very nearly the location of the manifold of Klebanov-Witten fixed points.

Let's return to our metaphor of the ball rolling in the potential. In the Klebanov-Witten model the renormalization group can be thought of as a ball rolling (with strong friction) in a landscape which has steep walls surrounding a deep, perfectly flat valley, representing the manifold of conformal fixed points. The ball rolls quickly into the valley, and stops. Once we add fractional branes, the only change is that the valley, instead of being perfectly flat, acquires a small tilt of order $1 / k$. The ball rolls quickly into 
the valley, comes nearly to a stop, then gradually drifts down the valley toward the regions of lower elevation and smaller $k$.

Our task, now, is to understand what happens during this drift. What does the theory do as its coupling constants slowly change? We will see that the answer is intricate and remarkable.

\subsection{Near the Boundary of the Valley}

Although there is no manifold of fixed points for this theory, there are isolated fixed points when two of the three couplings are zero. For instance, if $g_{k}=h=0$, then the theory is simply SQCD with $[k-1] M$ colors and $2 k M$ flavors. This is in the conformal window (for $k>3$ ) and has a Seiberg fixed point at which the fields $A_{r}, B_{u}$ have dimension

$$
\operatorname{dim} A_{r}=\operatorname{dim} B_{r}=\frac{3}{2}\left(1-\frac{[k-1] M}{2 k M}\right)=\frac{3}{4}\left(\frac{k+1}{k}\right)
$$

or equivalently

$$
\delta_{0}=\frac{3}{2 k}
$$

Then, from (4.2), when $0<g_{k}, \eta \ll 1$ we have

$$
\beta_{g_{k}}=-F\left(g_{k}\right)[6 M]<0, \beta_{g_{k-1}}=0, \beta_{\eta}=\frac{3 \eta}{k}>0
$$

to leading order in $1 / k$.

If instead $g_{k-1}=h=0$, then the theory is $S U(k M)$ SQCD with $2(k-$ 1) $M$ flavors; this is in the conformal window and its fixed point has

$$
\operatorname{dim} A_{r}=\operatorname{dim} B_{r}=\frac{3}{2}\left(1-\frac{k M}{2(k-1) M}\right)=\frac{3}{4}\left(\frac{k-2}{k-1}\right)
$$

or equivalently

$$
\delta_{0}=-\frac{3}{2(k-1)} \approx-\frac{3}{2 k} .
$$

Then we find, at this fixed point,

$$
\beta_{g_{k}}=0, \beta_{g_{k-1}} \approx-F\left(g_{k-1}\right)[-6 M]>0, \beta_{\eta} \approx-\frac{3 \eta}{k}<0 .
$$

So the signs are all reversed from the previous case.

Exercise: Determine the conditions for a fixed point and verify these statements. 
Let's now encode this information on a graph of the space of couplings, shown in Fig. 31. Suppose $g_{k}$ is zero and we are at the $S U(k[M-1])$ Seiberg fixed point, the upper dot on the graph. From Eq. (4.3), if $\eta$ is nonzero, then $\beta_{\eta}>0$; the theory flows back to the fixed point. But if $g_{k}$ is nonzero (and $\eta=0$ ) then the theory flows along the edge of the valley to the $S U(k M)$ fixed point, where $g_{k-1}=0$, the lower dot on the graph. Here Eq. (4.4) applies; the fact that $\eta$ is relevant at this fixed point is familiar, as it corresponds to $S U(k M)$ SQCD with $N_{f}=2(k-1) M<2 k M$, a case we studied in Sec. 1.4.

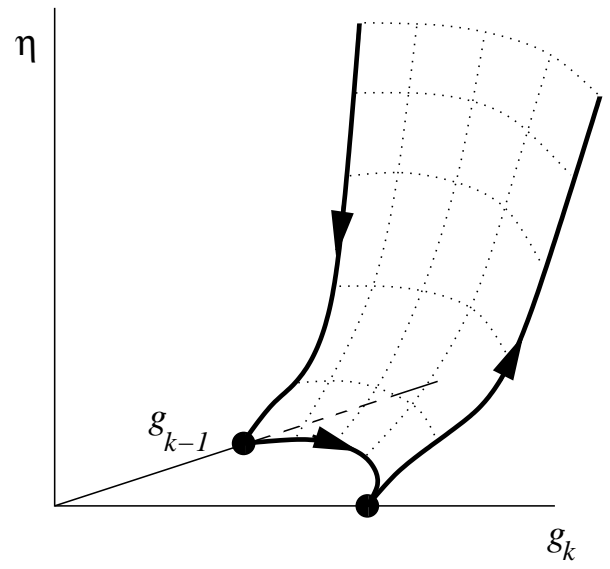

Figure 31. The drift along the boundary of the valley for nonzero $M$ and large $k$; compare with the space of conformal field theories in Fig. 18. Each dot represents a Seiberg SQCD fixed point.

What happens once $\eta$ is nonzero? As we saw in Sec. 1.8 and is shown in Fig. 12, the flow from small to large $\eta$ has an exactly-dual description in which we replace $S U(k M)$ with $S U([k-2] M)$ and $\eta$ with $\tilde{\eta} \sim 1 / \eta$. The endpoint of this flow is the Seiberg fixed point of $S U([k-2] M)$ SQCD with $2[k-1] M$ flavors. But at this fixed point $g_{k-1}$ is relevant, so if it is nonzero we obtain a flow to the fixed point of $S U([k-1] M)$ with $2(k-2) M$ flavors, as shown in Fig. 32. We can see that the process will repeat until $k \sim 1$.

The curves we've just discussed simply form the boundary of the valley. In a real flow, the gauge couplings and $\eta$ are never strictly zero, so the flow takes place within the valley, but away from its boundary, in a fashion 

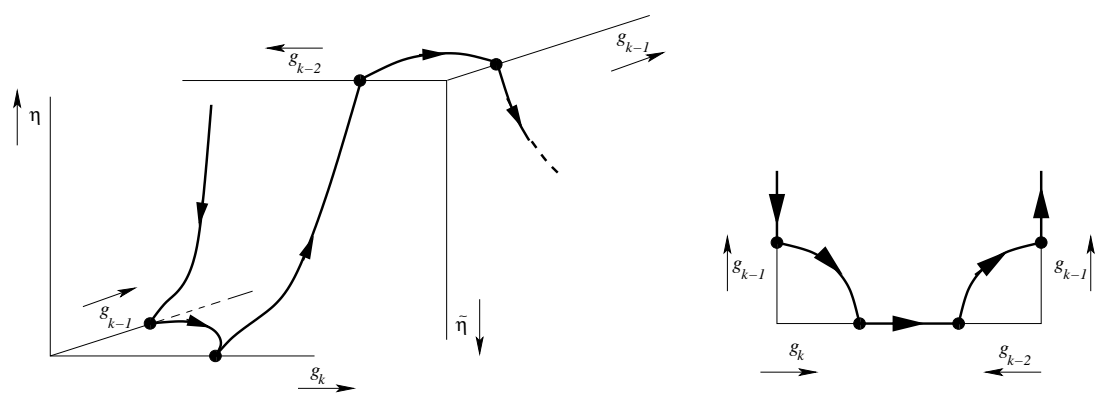

Figure 32. On the left, the flow along the boundary of the valley, extending Fig. 31 through the region appropriate for a Seiberg-duality transformation, as in Fig. 12. Each dot represents a Seiberg SQCD fixed point. Initially, the set of coordinates $g_{k}, g_{k-1}, \eta$ is appropriate; after the transformation, new coordinates $g_{k-2}, g_{k-1}, \tilde{\eta}$ should be used. On the right, the same image is shown looking down from above, as in Fig. 20, so that the $\eta$ (and $\tilde{\eta}$ ) coordinate is suppressed, and only the gauge couplings are retained.

better illustrated by the two curves added to Fig. 33. One of these curves (the dashed line) lies very close to the boundary, and passes very close to the Seiberg fixed points. When it reaches the vicinity of such a fixed point - $g_{k-1} \sim g_{k-1 *}$ and $\eta, g_{k} \ll 1$ - the motion along the curve might slow to a near-stop. To find out, let's check the beta functions. Near the fixed point, $\beta_{g_{k-1}}$ is of order $g_{k-1}-g_{k-1 *}$ (since it must vanish at $g_{k-1 *}$ ) but it also gets corrections from $\eta$ and $g_{k}$; by simple one-loop perturbation theory estimates, these will be of order $\eta^{2} / 8 \pi^{2}$ and $g_{k}^{2} k M / 8 \pi^{2}=\lambda_{k} / 8 \pi^{2}$. Meanwhile, from Eq. (4.3), the beta function for $\eta$ is of order $\eta / k$, while that for $g_{k}$ is of order $-6 M F\left(g_{k-1}\right)$; both of these are $1 / k$ smaller than their typical size. For the couplings $g_{k}$ and $\eta$ to remain small, and for $g_{k-1}$ to remain near its fixed value, for an extended range of energy, it must be that $\lambda_{k}=g_{k}^{2} k M$ - the 't Hooft coupling for the weakly-coupled $S U(k M)$ gauge group - is much less than 1 in this regime. Also, $\eta$ must be small.

So, if the curve corresponding to a particular flow dips down to a regime in which the 't Hooft coupling $\lambda_{k}=g_{k}^{2} k M \ll 1$ and $\eta \ll 1$, then there really is a range of energy scales in which the theory can be described as a conformal Seiberg fixed point of $S U([k-1] M)$ SQCD along with a weaklycoupled $S U(k M)$ gauge group and a small quartic coupling $\eta$. Conformal field theory for the $S U(k M)$ sector combined with conformal perturbation theory in $\lambda_{k-1}$ and $\eta$ would be an effective method for doing calculations 

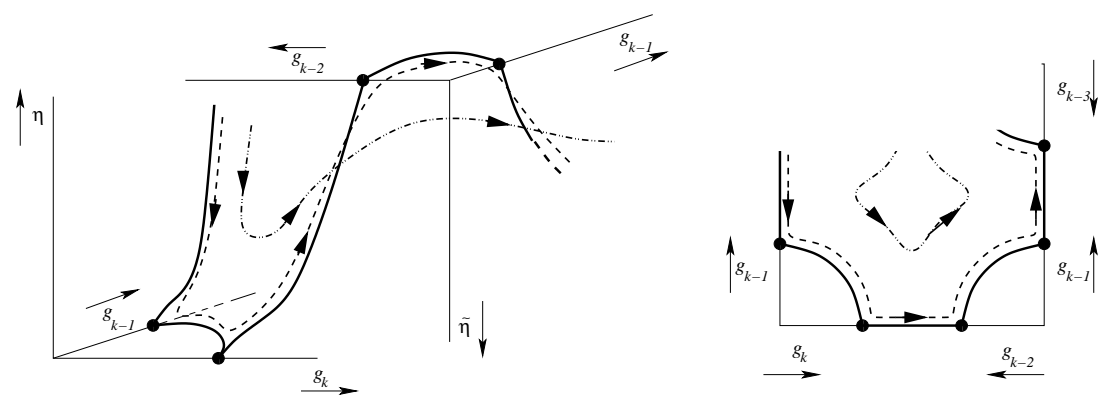

Figure 33. Two flows inside the valley, close to (dashed) and far from (dot-dashed) the boundary (solid) of the valley. The notation of the two figures is the same as for Fig. 32.

in this range.

Eventually, $g_{k}$ will increase to large values, with $g_{k-1}$ shrinking to zero; more precisely, $\lambda_{k-1}$ will become much less than 1 as the flow approaches the Seiberg fixed point at $g_{k} \rightarrow g_{k *}$. Near this fixed point, $\beta_{\eta}<0$, so after a long range of energy where the beta functions (4.4) are all very small, $\eta$ will begin to grow and will increase to large values.

But then, as we can see from the curve, we will find yet another range of slow motion in which the theory can be described as approximately conformal $S U([k-2] M)$ with weakly coupled $S U([k-1] M)$, two bifundamental fields, and small $\tilde{\eta}$. Logarithmic running of $\lambda_{k-1}$ would eventually destabilize this quasi-fixed point, leading us into yet another quasi-fixed point, that of $S U([k-1] M)$ with weakly coupled $S U([k-2] M)$ and small $\tilde{\eta}$. And this repeats, over and over. For this flow, there is really a "cascade," with a sequence of steps. The ball rolling down the valley nearly stops, then rolls to a new location and nearly stops, then rolls again to a new location, etc. The cascade from one fixed point to the next is represented by the curve in Fig. 34, and by the dashed curve in Fig. 33 and Fig. 35.

Does Seiberg duality apply exactly or approximately on this curve? The answer is that it applies exactly. To see this, we must bring two of our earlier arguments together. (1) If Seiberg duality is exact in the ultraviolet, it remains exact forever into the infrared. This is clear from Fig. 11. Once the two flows shown in Fig. 11 flow together, or more precisely, are brought together by taking a scaling limit, they simply aren't two flows anymore: they become a single flow with two descriptions. A single flow 


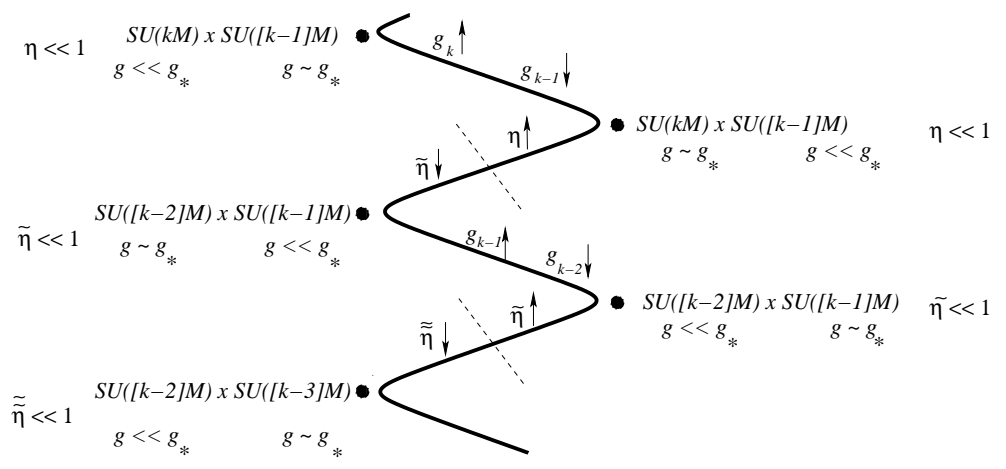

Figure 34. Near the boundary of the valley, the theory cascades from one Seiberg fixed point to another. First a flow occurs in which one gauge coupling shrinks from its Seiberg-fixed-point value to zero, while the other grows from zero to its Seiberg-fixed-point value. Next, $\eta$ grows from zero to infinity, necessitating a change of variables using an exact Seiberg-duality transformation; this is shown by a dashed line. From the dual point of view, $\tilde{\eta}$ shrinks to zero. The process then repeats.

cannot bifurcate in the infrared; that would make the renormalization group indeterminate. (2) The Klebanov-Witten model has exact Seiberg duality acting on its manifold of fixed points. The theory with fractional branes differs from the Klebanov-Witten model only by $1 / k$ corrections, and the duality cascade lies within the valley where exact Seiberg duality applies in the Klebanov-Witten model. Therefore Seiberg duality must apply up to at most $1 / k$ corrections. But in the ultraviolet, these disappear.

So Seiberg duality applies exactly in the ultraviolet, and therefore, from our previous point, it applies at all scales. Properly defined, the duality cascade is a single flow, with multiple (indeed, an infinite number of) descriptions, each of which has a limited range of usefulness.

If you don't like this argument (after all, it does give us an infinite number of colors in the ultraviolet) I'm happy to regularize it. Put an ultraviolet cutoff at $k=k_{0}$ large but finite. Seiberg duality applies approximately in the ultraviolet, with $1 / k_{0}$ corrections. But as is clear from the central diagram of Fig. 11, the duality applies better and better as the flow approaches the infrared; the two flows shown there become closer and closer to identical as the infrared is approached. This is because their difference is described by an irrelevant operator, whose effect decreases to zero in the 
infrared. So I can make the two flows arbitrarily close to one flow by only asking questions in the physical regime where $k \ll k_{0}$. Then the two flows, and their two descriptions, can be made identical, for fixed $k$, by taking $k_{0} \rightarrow \infty$. Again, this is because the difference between the two flows is an irrelevant operator, whose effect must vanish at any fixed energy scale as the cutoff at $k_{0}$ is removed.

In short, the duality cascade is about a single flow, with an infinite number of descriptions, one for every $k$, obtained from one another by application of an arbitrary number of exact Seiberg duality transformations. The flow given by the dashed curve in Fig. 33 and Fig. 35 moves from one approximate fixed point to the next, each with its own useful variables. We might wish to think about this flow as a "cascade of dualities," but in retrospect, this a rather poor choice of terminology. The dualities are not physical, while the cascade is physical. The cascade of the theory from one fixed point to another involves a journey from a region where one description of the theory is useful to a region where another is useful. Each description is valid at all scales, but is useful only in the region where the theory flows close to the $S U(k M) \times S U[(k \pm 1) M]$ Seiberg fixed points. ${ }^{19}$

We might be tempted to ask whether this theory really has an infinite number of colors. But we already know that this is not a meaningful question. We know that $S U(20) \mathrm{SQCD}$ with $N_{f}=28$ is exactly dual in some contexts to $S U(8)$ SQCD+M with $N_{f}=28$, so it must be that the number of colors cannot be computed from the answer to any physical question. Of course, the conformal anomalies of the theory are physically meaningful, and it is true that for theories of free particles, they do count the number of fields, and thus the number of colors. For interacting theories, however, the conformal anomalies do not tell us the number of colors. They give us only an estimate of how many colors a convenient (weakly-coupled!) description of the theory is likely to have. For the flow near the boundary of the valley of the duality cascade, the conformal anomalies themselves gradually cascade, decreasing sharply as the flow moves from the vicinity of one fixed point to the vicinity of another, then becoming nearly constant once the neighborhood of the new fixed point is reached, before jumping again as the

\footnotetext{
${ }^{19}$ Strictly speaking, it is possible that the $k$ th description breaks down above some $k$ dependent ultraviolet scale. However, each choice of variables can be used, in principle, for a simulation of the theory below this scale. Such a simulation will be valid to arbitrarily low energies, unless the renormalization group, which is unambiguous heading into the infrared, itself breaks down. Thus there is certainly an infinite number of descriptions in the infrared.
} 
flow moves onward. They do indeed scale as $k^{2} M^{2}$, but again, that doesn't tell us how many colors our description has to have. At best, they can only suggest to us that perhaps a $S U(k M)$ SQCD fixed point, with an extra weakly-coupled $S U([k \pm 1] M)$ gauge group, might lie nearby. Certainly they never count the number of fields in the model in a straightforward way, because at no energy scale are both gauge groups simultaneously weakly coupled.

\subsection{Far from the Boundary of the Valley}

All the statements made above apply when the flow lies near the boundary of the valley. But now look at the other curve in Fig. 33 and Fig. 35, represented by the dot-dashed line. This curve never lies near any fixed points. Nowhere are any of the couplings particularly weak, or particularly strong. We see that the $\lambda$ 's are always pretty large, and that $\eta \sim \tilde{\eta}$ is always of order one. We are nowhere near any well-understood fixed point, around which we might do perturbation theory. How can we analyze the theory when it flows along this curve?

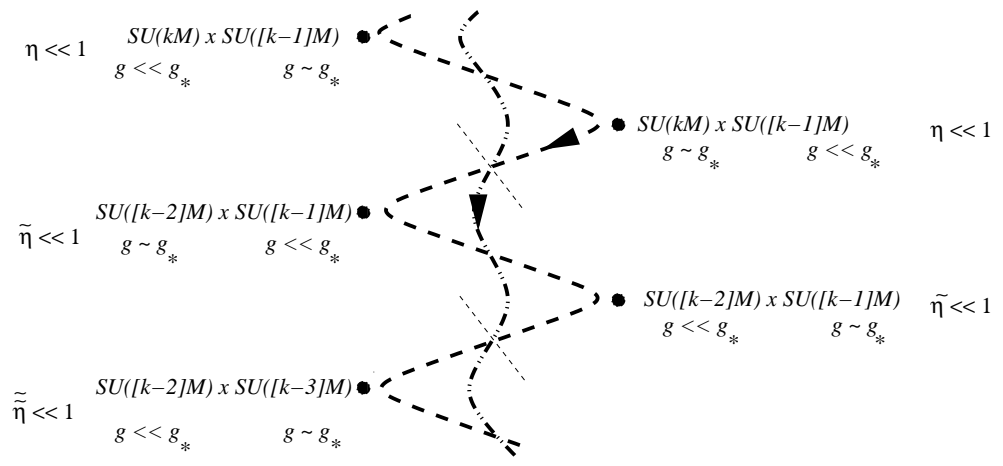

Figure 35. As in Fig. 34, showing the step-like character of flows (dashed) near the boundary of the valley, and the smooth character of flows (dot-dashed) far from the boundary.

To appreciate what this curve means, let's represent the data in another way, as we did for Fig. 20, by forgetting everything in the three-dimensional graph except the valley and compressing the graph into the plane of the gauge coupling constants. In Fig. 32, it is shown where the boundary of the 
valley lies; the right-hand diagram shows the above-mentioned projection. In Fig. 36 the boundary is shown again, extended to include another two steps in the cascade: one sees not only the gauge couplings for $S U(k M)$, $S U([k-1] M)$ and $S U([k-2] M)$ but also $S U([k-3] M)$ and $S U([k-4] M)$. Don't be misled! The valley does not actually close on itself, and the cascade is not an Escher staircase. Rather, think of this as a multi-sheeted graph, with a branch cut off to the left. The boundary continues onto the next sheets above and below the one shown.

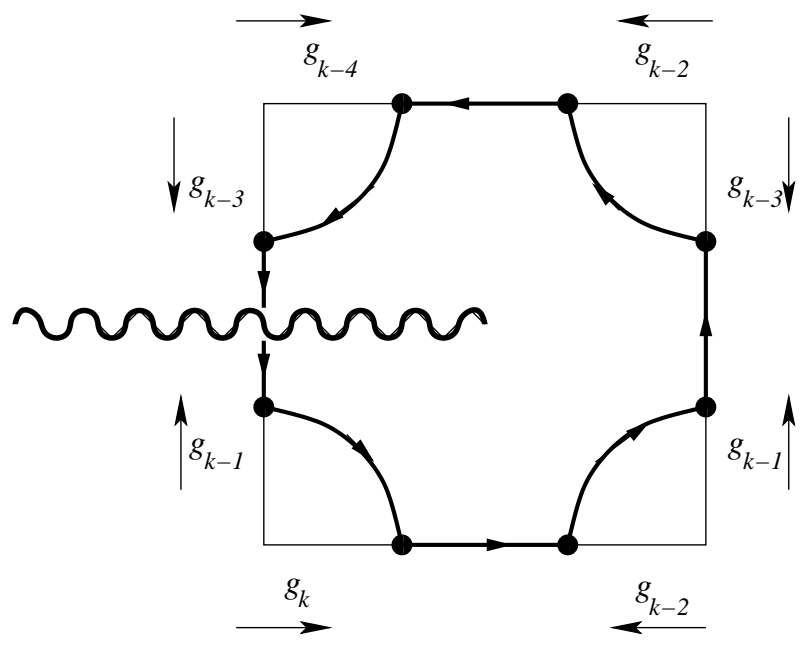

Figure 36. The boundary of the valley, viewed from "above" (i.e. with the $\eta$ coordinate(s) suppressed), shown for four steps of the cascade. Each dot represents a Seiberg SQCD fixed point. The cut at the left edge indicates that the space of couplings is viewed here as multi-sheeted, with flows emerging from the sheet above the one shown, and continuing on the sheet below.

In Fig. 37, I have extended the two continuous RG flows of Fig. 33, so you can see where they lie on the multi-sheeted graph of the valley. The outer one closely follows the boundary, and has multiple quasi-fixed points where two of the three couplings are small and the third one is almost fixed. The inner one blithely circles the origin without much structure. To see even more dramatically what is happening, I have drawn in the next graph, Fig. 38, the lines of constant $\eta$, which I don't know precisely but can estimate to order $1 / k$. (In this graph I have written the dual of $\tilde{\eta}$ as $\eta$, 
rather than stacking tildes ad infinitum as I have started to do in Figs. 34 and 35. This notation is quite reasonable conceptually, however, as is clear from the form of the figure.) The outer curve flows from $\eta \ll 1$ to $\eta \gg 1$ and back again, again and again during each step of the cascade. The inner curve, by contrast, sits at $\eta \sim 1$, sliding back and forth between $\eta>1$ and $\eta<1$ but never deviating far from it. Thus, the inner curve represents $\eta$ at a quasi-fixed point. Its beta function is zero on average and is always much less than one.

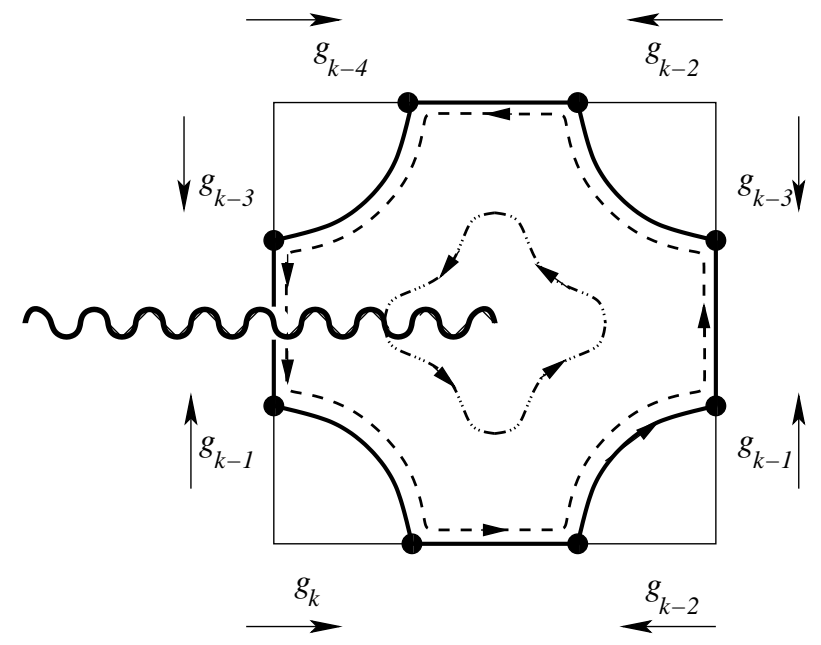

Figure 37. An extension of Fig. 33: two flows, near to (dashed) and far from (dotdashed) the boundary (solid) of the valley, viewed from "above," with notation as in Fig. 36.

Let us also consider what happens to the anomalous dimension $\gamma_{0}$. Lines of constant $\gamma_{0}$ will look similar to those of constant $\eta$. However, $\gamma_{0}$ never deviates much from $-\frac{1}{2}$ anywhere in the valley. Furthermore, on the inner curve, it is even better than that. The small $\beta_{\eta}$, combined with the fact that $\eta \sim 1$ on this curve, implies $\gamma_{0} \sim-\frac{1}{2}$ to very good accuracy - presumably much better accuracy than on the outer curve, where it deviates from $-\frac{1}{2}$ by order $1 / k$.

Finally, let's look at the lines of constant $\tau_{ \pm}$, the parameters of the space of theories in the Klebanov-Witten case. Comparing Fig. 39 and Fig. 40 with Fig. 37, we see that the flow lines tend to lie at nearly constant $\tau_{+}$ 


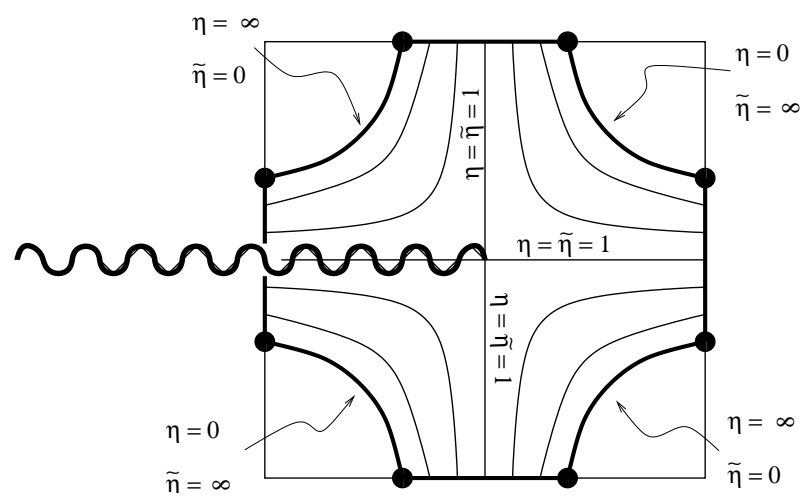

Figure 38. Lines of constant $\eta$ and $\tilde{\eta}$ in the valley; notation as in Fig. 36.

and that the flow involves a continuously decreasing $\tau_{-}$. In fact, we can imagine that near the center of the diagram there might exist a scheme where the different flows in the valley would be indexed by $\tau_{+}$, and the flow along each line would be parametrized by $\tau_{-}$.

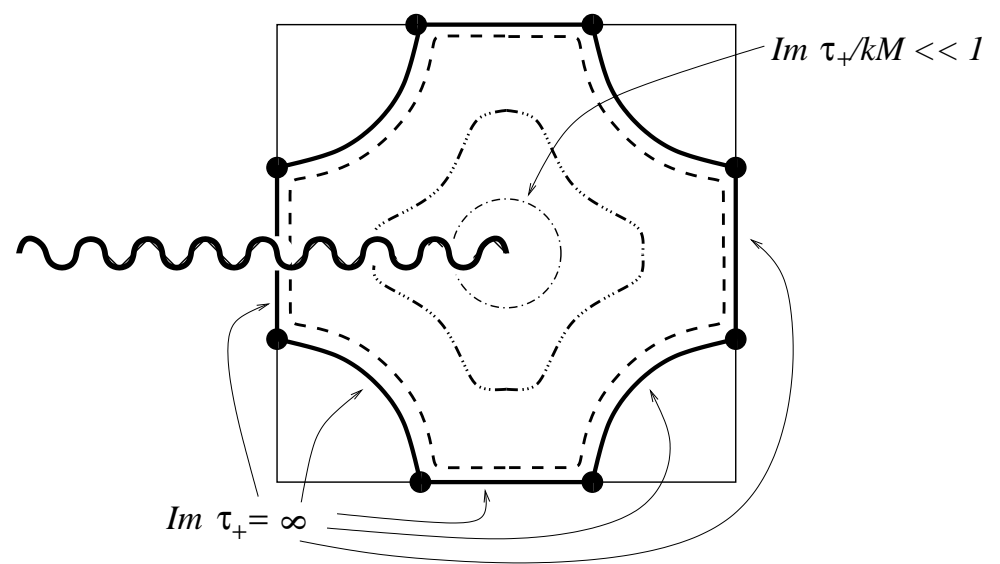

Figure 39. Lines of constant $\tau_{+}$in the valley; notation as in Fig. 36.

Now, it should be clear where we are heading. In the region around the dot-dashed curve, none of the field theory descriptions is particularly 


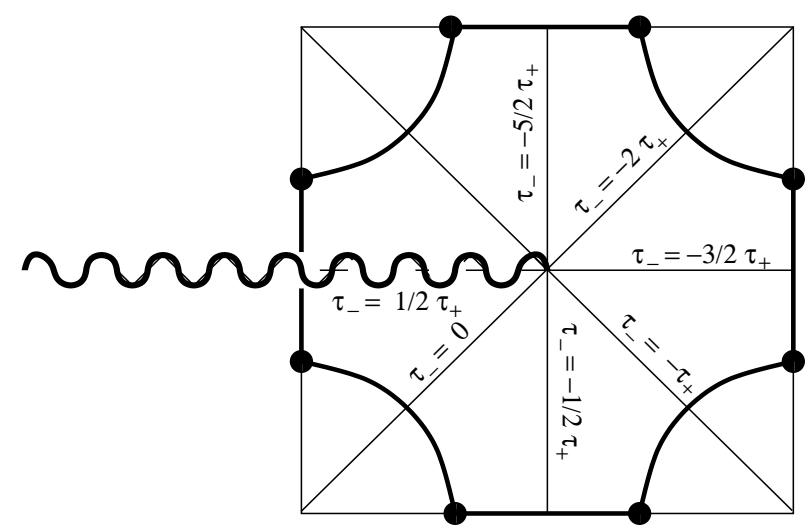

Figure 40. Lines of constant $\tau_{-}$; reflection symmetries under exchanging the two gauge groups or under $\eta \rightarrow 1 / \eta$ fix the ratio $\tau_{-} / \tau_{+}$on the lines shown, up to a shift of $\tau_{-}$by $\tau_{+}$. Notation is as in Fig. 36 .

useful, but the string theory description of the flow is particularly simple there. The quantity $\tau_{+}$is related to the string coupling, given by the dilaton, which is constant. The center region is where $\tau_{+} \sim 1 / g_{s}$ satisfies $g_{s} k M \gg 1$, which is the condition that the string theory be propagating on a space of small curvature. This in turn assures that a supergravity approximation to the string theory is valid. For $g_{s} k M \ll 1$, as is true (for any $k$ ) near the boundary of the valley $\left(\tau_{+} \rightarrow i \infty\right)$, the RG flow is choppy, involving a stepping from one quasi-fixed point to another; this is the regime where the calculations (4.1)-(4.2) and the ensuing conclusions are valid. But in the region where supergravity is a good approximation to the string description of the flow, the flow is smooth, never approaching an approximate fixed point, though still flowing slowly, at least for large $k$.

Again, when the flow is smooth and lies far from the boundary of the valley, none of the $S U(k M) \times S U[(k-1) M]$ descriptions of the theory are particularly useful. But they are still present. There are two direct ways to see this. First, we can describe the smooth flow, in any particular energy range, as follows: find a choppy flow nearby (by increasing $\operatorname{Im} \tau_{+}$, holding other couplings fixed), describe the choppy flow by using any of the simple variables inherited from the nearest Seiberg fixed point, and then go back to the smooth flow by reducing $\operatorname{Im} \tau_{+}$again. If Seiberg duality applies to the choppy flow, and tells us what operator corresponds to the coupling $\tau_{+}$ 
in two different descriptions, then it tells us, in two different descriptions, how to get from the choppy flow back to the smooth one, bringing the two different descriptions with it. Second, all flows except those with $\tau_{+} \rightarrow i \infty$ reach the supergravity regime in the ultraviolet, since $k \rightarrow \infty$ there. Any flow with $g_{s} M \ll 1$ will have a critical value of $k=k_{c} \sim 1 / g_{s} M$ where for $k \gg k_{c}$ the supergravity description is useful, while for $k \ll k_{c}$ the field theory descriptions become useful. Essentially, all flows gradually spiral out toward the boundary, and those with $g_{s} M \ll 1$ actually reach its vicinity at $k \sim 1 / g_{s} M$. So for any finite $\tau_{+}$with $M / \operatorname{Im}\left(\tau_{+}\right) \ll 1$, the field theory descriptions are eventually going to be necessary for describing the flow. By adjusting $\tau_{+}$, we adjust the critical $k_{c}$ where this occurs, and thus we can continuously slide from a flow for which a particular field-theory description is useful to one in which it is not, without in any way reducing the validity of that description.

Meanwhile, the question "how many colors does the theory have?" is even less meaningful here than on a choppy flow, since no field-theory description, with any number of colors, is useful for curves in the supergravity regime. But as before, we can turn to the conformal anomalies; these provide a measure of the number of colors that nearby choppy flows would need if they were to be described usefully using a choice of field-theory variables. We will see below that these decrease smoothly, without jumps, and are of order $k^{2} M^{2}$.

In summary, the entire valley, from its choppy boundary to its smooth interior, can be described in an infinite number of ways, using an infinite number of choices of variables, each involving an $S U(k M) \times S U[(k-1) M]$ gauge group with bifundamental matter, along with an additional string theory description to boot. This statement applies to all the flows within the valley, which form a one-parameter family indexed by $\tau_{+}$, with $\tau_{-}$ the coordinate along the flow. Each of these flows gradually descends the valley, beginning with a smooth flow in the regime where supergravity is valid. The flows gradually move out toward the boundary as they descend. A flow with $g_{s} M \gg 1$ remains always within the supergravity regime, and its flow is always smooth; but a flow with $g_{s} M \ll 1$ will transition out of the supergravity regime at $k \sim 1 / g_{s} M$, and thereafter will begin to cascade in a choppy fashion, passing through regimes in which one or another field theory description will be useful. 


\subsection{SUGRA and the Flow at Large $k$}

We will now construct the supergravity description of the flow at large $g_{s} k M$. For small $g_{s} M$, the description will be good only for $k \gg k_{c} \sim$ $1 / g_{s} M$, but for large $g_{s} M$ it will describe the entire flow, start to finish, ultraviolet to infrared.

Since the flow lies far from the boundary of the valley, it flows smoothly, without jumps. How can we describe it using supergravity? What might we guess, if we were naive? The fact that the conifold is deformed plays no role here, since we are far from the region of deformation. For $M=0$ we have

$$
\left(\frac{1}{4 \pi^{2} \alpha^{\prime}}\right)^{2} \int_{T^{11}} F_{5}=N=\frac{4 R^{4}}{27 \pi g_{s}} .
$$

Here we would expect this integral to equal the local value of $N$, namely $k(r) M$, where I have indicated explicitly that $k$ is a function of the momentum scale in the gauge theory, and thus of $r$ in the supergravity. In other words,

$$
\left(\frac{1}{4 \pi^{2} \alpha^{\prime}}\right)^{2} \int_{T^{11}} F_{5}=k(r) M
$$

which implies the $A d S$ radius gradually must change as $k(r)$ changes,:

$$
R^{4}(r) / \alpha^{\prime 2} \approx \frac{27 \pi}{4} g_{s} k(r) M .
$$

We might guess this captures everything, and that we need merely substitute $R(r)$ into the $A d S_{5} \times T^{11}$ metric

$$
\begin{aligned}
d s^{2} & =R^{2}(r) d s_{A d S_{5}}^{2}+R^{2}(r) d s_{T^{11}}^{2} \\
& =\frac{r^{2}}{R^{2}(r)} d x^{2}+\frac{R^{2}(r)}{r^{2}}\left(d r^{2}+r^{2} d s_{T^{11}}^{2}\right) .
\end{aligned}
$$

This space is said to be a warped version of the Klebanov-Witten metric.

Now, what is $k(r)$ ? The beta function for $\tau_{-}$near $\tau_{-}=0$, where $g_{k} \approx g_{k-1} \equiv \bar{g}$, is

$$
\beta_{\tau_{-}} \approx \beta_{\frac{4 \pi}{g_{k}^{2}}}-\beta_{\frac{4 \pi}{g_{k-1}^{2}}} \approx \frac{1}{2 \pi} \frac{6 M-2 M \delta_{0}}{1-\frac{\bar{g}^{2}}{8 \pi^{2}}} .
$$

Since $\delta_{0}$ is always of order $1 / k$ in the valley, it can be neglected. We see, then, that $\tau_{-}$has a beta function of the same form as the beta function 
for pure $\mathcal{N}=1 S U(M)$ supersymmetric Yang-Mills theory itself: it is proportional to $M$, not $k M$. The solution to this equation is a logarithmically running $\tau_{-}$. For the string theory this implies, using Eq. (2.6),

$$
\frac{1}{4 \pi^{2} \alpha^{\prime}} \int B_{2} \sim \frac{\tau_{-}}{2 \tau_{+}}=\frac{3 g_{s} M}{2 \pi} \ln \left(r / r_{s}\right)
$$

where $r_{s}$ is where $k$ reaches 0 . For every step down the cascade, $k$ decreases by $1, \tau_{-}$decreases by $2 \tau_{+}$, and thus $\frac{1}{4 \pi^{2} \alpha^{\prime}} \int B_{2}$ also decreases by 1 . We may therefore guess that

$$
k(r)=\frac{1}{4 \pi^{2} \alpha^{\prime}} \int_{S^{2}} B_{2}=\frac{3 g_{s} M}{2 \pi} \ln \left(r / r_{s}\right) .
$$

As $d k / d r \propto 1 / r$, the speed of the cascade increases in the infrared; note also its speed is proportional to the 't Hooft coupling $g_{s} M$.

Meanwhile, $F_{3} \propto M \omega_{3}$, such that

$$
\frac{1}{4 \pi^{2} \alpha^{\prime}} \int_{S^{3}} F_{3}=M
$$

This should be constant, so we can use $F_{5}=B_{2} \wedge F_{3}$ (plus another term to make it self-dual) to infer that $\left(\frac{1}{4 \pi^{2} \alpha^{\prime}}\right)^{2} \int F_{5}=3 g_{s} M^{2} \ln \left(r / r_{s}\right)$. Consequently, from Eqs. (4.5) and (4.7), $R^{4}(r)=(81 / 8)\left(g_{s} M\right)^{2} \alpha^{2} \ln \left(r / r_{s}\right)$. Interestingly, this goes as $\left(g_{s} M\right)^{2}$, not as $g_{s} M$. This difference from noncascading models impacts a number of scaling relations and leads to confusion if one forgets about it.

Finally, from the curves we have been drawing we expect we should require that the dilaton be constant, which can only be true if its source, $F_{3}^{2}-H_{3}^{2} / g_{s}^{2}$, is zero. You can easily check this is true! And in fact, this naive argument turns out to be almost precisely correct, as shown in Klebanov and Tseytlin [25], as long as both $k \gg 1$ and $g_{s} k M \gg 1$. The only difference from what I have suggested here is a small (order-1/k) constant shift in $R^{4}$ relative to $F_{5}$.

We can now check that, as claimed earlier, the conformal anomalies are decreasing as $k^{2} M^{2}$. This is essentially trivial. The conformal anomaly for $\mathcal{N}=4 S U(N)$ Yang-Mills is a constant times $N^{2}$. The constant is independent of the gauge coupling, so the conformal anomaly counts the number of degrees of freedom in the free theory. We can compute the anomaly from the $A d S_{5}$ metric [27] or more easily from the volume of the $S^{5}$ that appears in the gravitational metric [16]. For the Klebanov-Witten model the anomaly is larger by a factor of $27 / 16$; this can be computed in the field theory at the Seiberg fixed points, or computed far from the 
valley's boundary using the fact that the volume of the $T^{11}$ space is smaller by $27 / 16$ than that of the $S^{5}[16]$. Note this does not mean the number of fields in the theory is equal to $27 / 16$ times the number of fields in $\mathcal{N}=4$ $S U(N)$ Yang-Mills, as you can easily check; the anomaly is not directly counting the number of fields. Finally, we have seen that all we have to do to write the supergravity description of the duality cascade at large $r$ is to replace $N$ in the Klebanov-Witten model with $N(r)=k(r) M$. Thus the anomaly is proportional to $[k(r) M]^{2}$, as we claimed, times the above mentioned factor of 27/16. Again, one cannot read off, from this formula, exactly how many colors would be useful in a description of this or nearby flows. One can only expect that the number of colors in a useful description is probably decreasing roughly as $k(r) M$.

We should also address a nagging issue that may have bothered the reader. Is it really sensible to extrapolate the flows under discussion up to the extreme ultraviolet, where $k \rightarrow \infty$ and the number of colors diverges? A field theory is defined normally by holding the number of degrees of freedom fixed and taking an ultraviolet cutoff to infinity. Can we really take the number of degrees of freedom to be infinite? Well, there is no law which forbids us to take the number of degrees of freedom to gradually increase as the cutoff scale is raised. As long as there are well-defined Green functions for operators that are sensible in the limit, the theory is consistent. Now that we have the supergravity description of the large- $k$ regime, we can check that the theory, though it has an infinite number of degrees of freedom, still has many finite and computable amplitudes satisfying the usual constraints of local field theory [28]. In particular, in the ultraviolet many amplitudes (properly normalized) gradually approach those of the Klebanov-Witten model, with only very slow logarithmic running to violate the power-law Green functions expected in conformal theories. These issues deserve further investigation, but there is plenty of evidence that these theories are well-behaved. ${ }^{20}$

In the infrared, on the other hand, something drastic must happen as $k \rightarrow 0$. If $g_{s} M \ll 1$, then supergravity breaks down at $k \sim 1 / g_{s} M$; we have little hope of describing the transition regime, though at lower energies our

\footnotetext{
${ }^{20}$ Other classes of theories of similar type are trivial to construct. For instance, consider an $S U(N) \mathcal{N}=4$ gauge theory broken to $U(1)^{N-1}$ by a scalar whose expectation value is $\left\langle\Phi_{1}\right\rangle=\operatorname{diag}\left\{v_{1}, v_{2}, \ldots, v_{N}\right\}$, where $v_{1}<v_{2}<\cdots<v_{N}$. The $v_{n}$ are free parameters and can be chosen arbitrarily. Now take the limit $N \rightarrow \infty$. By adjusting the dependence of the $v_{n}$ on $n$, many different $S U(\infty)$ gauge theories, with correspondingly different metrics in their supergravity dual descriptions, may be obtained.
} 
field-theory methods will apply. But for $g_{s} M \gg 1$, supergravity should apply all the way to the infrared. In this case, we should complete our metric, accounting for the fact that the conifold is deformed as well as warped in the infrared. We will round out our story with some general words about this computation.

\subsection{The End of the Cascade}

Now we must match our complete understanding of the large $k$ region onto the end of the flow as $k \rightarrow 0$. We've already understood what will happen for $k=3,2,1,0$ from our earlier field theory analysis. I've drawn a graph using the technique I introduced earlier, though I've made some changes. Note the boundary of the valley is quite different. This is partly to account for the fact that $1 / k$ corrections are not small. More importantly, for $k \leq 4$ the $S U(k M)$ theory with $2(k-1) M$ flavors is no longer in the conformal window. Instead it lies in the free magnetic phase, so there are no Seiberg fixed points in this regime. Instead, the infrared fixed points for $k=4,3$ lie at $g_{k-2} \rightarrow$ zero, and for $k=2$ the dual theory has no gauge coupling (though its low-energy confined bound states have other infraredfree couplings which I have indicated schematically as $y_{0}$.) At the top of the graph, the only remaining light degrees of freedom of the theory are those of pure $S U(M)$ Yang-Mills.

Our field theory calculations for $k \leq 3$ were always performed in regions where one of the gauge couplings was small. This was necessary to make the calculations easy. ${ }^{21}$ Thus we actually computed the RG flow appropriate to the outer curve in Fig. 41, the choppy flow between quasi-fixed-points. In short, we were working at $g_{s} M \ll 1$.

But we now want to know what happens in the supergravity regime. The inner curve in Fig. 41 indicates what we should expect. Both curves in Fig. 41 end in a confining and chiral-symmetry breaking phase, and their paths look reasonably similar, but actually they are very different. The issue is the rate of flow. Before flowing into the confining region, the outer curve gets stuck for an extended period in the preceding corner. In fact, the range of energy $\mu_{H}>\mu>\mu_{L}$ during which it remains in this corner is exponentially large: $\mu_{H} / \mu_{L} \sim \exp \left(8 \pi^{2} / g_{1}^{2} M\right)$, where $g_{1}$ is the minimum value of $g_{1}$ in this corner. This follows simply from oneloop perturbation theory in $g_{1}$; the theory is essentially pure Yang-Mills,

${ }^{21}$ The calculations should be tractable, though difficult, even without taking this limit. To my knowledge no one has looked seriously at this problem. 


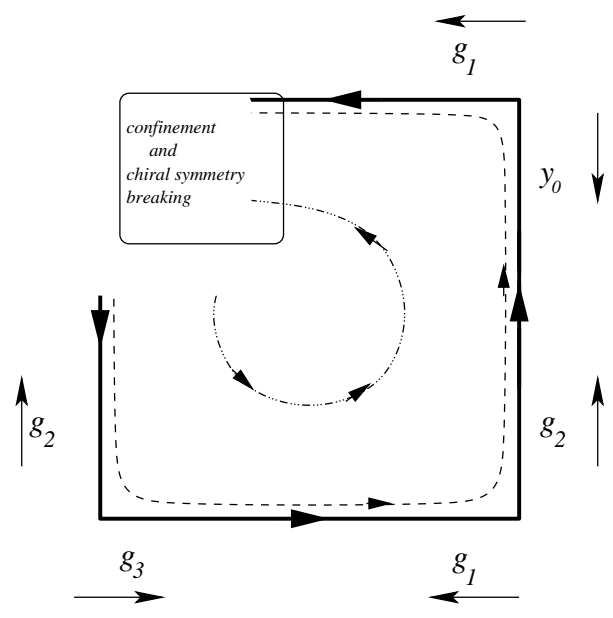

Figure 41. The last steps in the cascade. The flow on the outer curve is choppy, remaining for an extended period in each corner. The flow on the inner curve is smooth and steady. The endpoints of the flows both exhibit confinement and chiral symmetry breaking, but in other respects they are very different.

once the $S U(2 M)$ group confines and only $S U(M)$ remains (along with a neutral Goldstone multiplet.) But the inner curve flows smoothly into the confining region, with no exponential hierarchies. At no point does the theory resemble pure Yang-Mills, even though it is in the same "universality class." Its extreme infrared structure is the same, but above the infrared lie many massive partices. For instance, the $Z^{\text {adj }}$ fields which appeared in the $S U(2 M) \times S U(M)$ analysis - massive adjoint scalars and fermions of $S U(M)$, which are not present in pure $\mathcal{N}=1$ Yang-Mills theory - lie close to the confinement scale. So on the inner curve we do not ever find, at any scale, pure $\mathcal{N}=1$ Yang-Mills; all we have is a theory with some similar features. We can study confinement and chiral symmetry breaking using supergravity, but this magical ability doesn't come for free — we don't get to study it in the theory of greatest interest. ${ }^{22}$

How should we find the supergravity? We will begin with a simple

\footnotetext{
${ }^{22}$ And indeed it is clear why we cannot: real-world QCD lies outside the supergravity regime, and any theory which resembles it, such as $\mathcal{N}=1$ Yang-Mills, will also lie outside the supergravity regime. Only in very limited circumstances might we gain some deep insight into QCD or $\mathcal{N}=1$ Yang-Mills from supergravity. The trick is to identify those circumstances!
} 
ansatz: let us assume that the metric continues to be a warping of a simple metric, of the form

$$
d s^{2}=h(r)^{-1 / 2}\left(d x^{\mu}\right)^{2}+h(r)^{1 / 2} d s_{6}^{2}
$$

where $d s_{6}^{2}$ is the metric of the deformed conifold. In the absence of the deformation, or equivalently at large $r$, we found earlier that $h(r)=R^{2}(r) / r^{2}$, where $R^{4}=(81 / 8)\left(g_{s} M \alpha^{\prime}\right)^{2} \ln \left(r / r_{s}\right)$; now presumably it will get some correction at $r \sim r_{s}$ and will not go negative. We will also assume the dilaton is still constant. Now, this turns to be a good guess! The ensuing computation is too laborious to present here, and it is well-described in the original paper, and more precisely in the Les Houches Lectures written up by Herzog, Klebanov and Ouyang [29]. But here are some facts. The metric is indeed sensible, with $h(r)$ nonsingular and approaching a nonzero constant as $r$ goes to a minimum value $r_{\min }$. The resulting space is everywhere weakly-curved and nonsingular, has no horizon, and is fully described within supergravity. ${ }^{23}$

We expect that the theory has the same qualitative features as $\mathcal{N}=$ 1 supersymmetric Yang-Mills, modulo the presence of a single massless Goldstone supermultiplet. Let's go through the list.

- A discrete spectrum, with spacing of order $m$.

When the space is cut off at $r_{\min }$, and has no horizon, it is effectively made compact (since gravity tends to pull things in from the boundary.) A five-dimensional space with one compact direction (or more appropriately, a ten-dimensional space with six compact dimensions) will give a discrete four-dimensional spectrum, with modes carrying wave functions such as shown in Fig. 42. Let's call the typical spacing between masses of low-lying states $m$.

- Confinement (and associated strings carrying flux)

\footnotetext{
${ }^{23}$ This is in contrast to the $\mathcal{N}=1^{*}$ theory investigated by Polchinski and Strassler [30]. The confining vacuum found there is a supergravity solution with an NS5 brane in it. While this is a perfectly reasonable solution, it is difficult to work with string theory in the presence of a single NS5 brane, at least for modes close to the 5-brane. Since low-lying hadrons are of this type, there would be some problems calculating low-energy processes in the $\mathcal{N}=1^{*}$ model. Also, the exact metric for this theory remains unknown.
} 

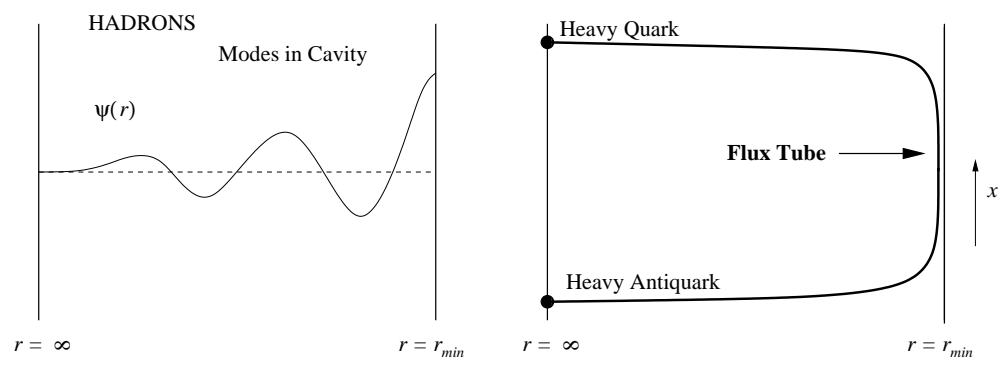

Figure 42. On the left, the wave function for a cavity mode in five dimensions that represents a typical four-dimensional hadron. On the right, a string with both ends on the boundary and its middle lying at $r=r_{\text {min }}$ represents an infinitely heavy quark and antiquark joined by an object of constant energy per unit length: a confining flux tube.

A Wilson loop corresponds to a string with its ends on the boundary, as shown in Fig. 42. As long as its tension does not vanish anywhere, it will give an area law. And the tension cannot vanish, because it is proportional to $h(r)$, which is nowhere zero (in contrast to $A d S_{5}$ where it vanishes on the horizon at $r=0$.) The string tension is larger than $m^{2}$ by the ratio $R^{2} / \ell_{s}^{2}=g M$, not $\sqrt{g M}$ as in $\mathcal{N}=1^{*}$. (Here we see the effect of the unusual dependence of $R$ on $g M$.)

- Chiral symmetry breaking $(a\langle\lambda \lambda\rangle$ condensate spontaneously breaks $\mathbf{Z}_{2 M}$ to $\mathbf{Z}_{2}$, since now only $\alpha=0, \pi$ is an unbroken symmetry)

This follows directly from the appearance of the deformed conifold metric, which depends on the parameter $\epsilon$. This parameter is left unchanged only by the $\mathbf{Z}_{2}$ subgroup of the classical $U(1)_{\mathcal{R}}$.

- A moduli space with $M$ identical degenerate branches, with $\langle\lambda \lambda\rangle=$ $e^{2 \pi i n / M}\left(\Lambda_{0}^{3 M}\right)^{1 / M}, n=0, \ldots, M-1$ (since as with any spontaneously broken symmetry $G \rightarrow H$, the vacua must form a representation of $G / H \approx \mathbf{Z}_{2 M} / \mathbf{Z}_{2} \approx \mathbf{Z}_{M}$.)

The fact that $U(1)_{\mathcal{R}}$ is broken to $\mathbf{Z}_{2 M}$ can be derived by studying 
the anomaly of the theory, as worked out carefully in Klebanov, Ouyang and Witten [19]. Once this is established, the $\mathbf{Z}_{2 M}$ rotations of the phase of the parameter $\epsilon$, modulo the $\mathbf{Z}_{2}$ under which it doesn't change, give $M$ different possible choices of $\epsilon$ for a fixed $|\epsilon|$.

- Domain walls which separate one vacuum from the next (which are required by the previous condition); it turns out these are BPS saturated (Dvali and Shifman, 1997).

These are D5-branes wrapped on the finite-radius $S^{3}$ at the tip of the deformed conifold. With three dimensions on the $S^{3}$, these branes have a $2+1$ dimensional world-volume appropriate to a field theory domain wall. D5-branes are magnetic sources for $C_{2}$, so $d C_{2}$ is nonzero, leading to a shift in $C_{2}$ as we cross a D5-brane. This is equivalent $[19,29]$ to a shift in the phase of the deformation parameter $\epsilon$.

Thus our solution reproduces the qualitative physics of $\mathcal{N}=1$ Yang-Mills theory, with the appropriate adjustments for the extra Goldstone mode. ${ }^{24}$

\subsection{Goldstone modes}

One might ask whether the massless Goldstone mode (and its scalar superpartner) makes this theory profoundly different from pure $\mathcal{N}=1$ YangMills theory. This issue has not been fully explored, but to my mind it has two answers.

On the one hand, important details of phenomenology are different in certain processes; certain particles that are stable in $\mathcal{N}=1$ Yang-Mills will be able to decay here, new annihilation channels open up in hadronantihadron scattering, etc. Certain Green functions and scattering processes will be quite different. This is very important if one wishes to use

\footnotetext{
${ }^{24}$ One can also look at dibaryons here, using D3-branes wrapped on the $S^{3}$. In field theory one can see that in $S U(N) \times S U(N-M)$ these objects carry $M$ hanging indices, which can be joined via confining flux tubes to $M$ external quark sources. In short, they act as baryon vertices in the pure $S U(M)$ theory. This is also seen in the supergravity. The $F_{3}$ flux from the $M$ D5-branes generates $M$ units of string charge on the wrapped D3-brane, which must then be joined to $M$ external strings in order that the wrapped brane have finite energy.
} 
the duality cascade for realistic model building. (However, in realistic cases one can expect the $U(1)_{B}$ to be gauged, which replaces the massless Goldstone boson [and its superpartners] with a massive vector boson [and its superpartners], alleviating some of the phenomenological problems. It also allows for finite tension cosmic strings [31].)

On the other hand, the Goldstone mode is odd under charge conjugation $\left(A_{i} \leftrightarrow B_{i}\right)$ which is an exact symmetry of the duality cascade. For Green functions of operators which are even under this $\mathbf{Z}_{2}$ transformation, or if we consider the scattering of hadrons for which both all initial state hadrons and all final state hadrons are even, then the Goldstone modes cannot appear in any tree-level string theory diagram. In other words, they cannot appear in any planar graph in the field theory with even external operators or states. In such processes, the Goldstone modes must be created in pairs, so they can only appear in loops, giving $1 / M^{2}$ corrections.

In short, if one's interest is in learning about the properties of matrix elements of $\mathcal{N}=1$ Yang-Mills, or of confining gauge theories in general, it is straightforward to restrict oneself to matrix elements where the Goldstone modes make no contribution at leading non-vanishing order. However, more care must be taken in building models of particle physics with this particular theory, as this Goldstone mode could be more problematic (or useful) there. This remains to be explored further.

Finally, let us recall that we earlier encountered another simple method by which we could obtain more interesting Goldstone modes, with the same quantum numbers as pions. In Sec. 3.2 we added an integer D3-brane to probe the space. Looking back, we see this extra brane makes the cascade of the form $S U(k M+1) \times S U([k-1] M+1)$, with $k$ still shifting by 1 in each step of the cascade. The extra brane has two main effects. First, confining flux tubes will now be able to break, as in QCD, because at the last step $(k=1)$ there is matter in the fundamental representation of $S U(M+1)$. Also, as we saw following Eq. (3.6), the angular coordinates of the position of the extra D3-brane are pions from the coset $[S U(2) \times S U(2)] / S U(2)$ or $[S U(2) \times S U(2)] / U(1)$, depending on the D3-brane location. (It also appears that there is no breaking of baryon number and no Goldstone mode, but this remains to be verified.) If we add the electroweak theory to this model by gauging an $S U(2) \times U(1)$ subgroup of the $S U(2) \times S U(2)$ global symmetry, we can obtain the standard model symmetry-breaking pattern through strong-coupling dynamics. This is a version of technicolor, and it is natural to use it for model-building, with application to particle physics and the Large Hadron Collider. Research of this sort is ongoing, 
and is closely related to the work being done on Higgsless models [32].

\subsection{Final Thoughts}

Now, what is this all good for?

Any calculable toy model of four-dimensional confinement and chiral symmetry breaking (both discrete and continuous) is a very good thing. To my knowledge, this is really the first example of its kind. In principle, everything in this theory that resides in the supergravity regime (and many stringy effects too) can be calculated semi-analytically. Hadron spectra, scattering amplitudes, matrix elements - you want it, we can compute it.

Can we use this to learn something about QCD? Many QCD processes are already calculable, using the bona fide QCD Lagrangian, and these new methods aren't needed. These include processes truly dominated only by ultraviolet physics, which are approachable using perturbation theory at weak QCD coupling, and processes in which the only long-distance (i.e. infrared, and therefore nonperturbative) physics can be related to Euclidean Green functions (which can be calculated using a computer.) However, there are many processes which are both nonperturbative and fundamentally Minkowskian, including for example certain types of Regge physics, quark-antiquark fragmentation into hadrons, quark-hadron duality, and semi-inclusive or exclusive hadron scattering. For these processes, no calculational methods exist, and a calculable toy model could prove useful.

But I certainly do not expect the models described above to generate numbers that we can compare with experiment. These are toy models of QCD: for one thing, they are supersymmetric, and we can only calculate their properties for $M$ and $g^{2} M$ large. Generally, toy models don't give predictions; at best they give insights and new methodology which can then be applied to the real model, hopefully leading eventually to predictions.

Could we do better if we could find a version of the duality cascade with real-world QCD as its low-energy limit? We would need to break supersymmetry and add quarks; see for example [33, 34, 42, 35, 36, 37]. Suppose we succeeded; would that improve our situation? Somewhat, but not too much. At small $g^{2} M$, a theory such as the duality cascade would be similar in the infrared to QCD, but would not be under theoretical control. Even in a supersymmetric theory, the field theory methods of Seiberg et al. [1, 3, 22] just aren't quite powerful enough to compute quantities of greatest interest, such as the hadron spectrum, scattering amplitudes, fragmentation, etc. To compute anything we must be at large 
$g^{2} M$, where supergravity methods are accurate, but this is a bad place to look for real-world QCD. Even if we could calculably embed QCD itself into a model which could be continued to large $g^{2} M$, the theory in the (super)gravity regime, by construction, would be very different from the real world. In particular, the small five-dimensional curvature, required for supergravity to be useful, also unavoidably implies all sorts of non-QCDlike behavior [38, 39, 30, 40, 41]: (1) low-spin hadrons with $m^{2}$ much less than the confining string tension; (2) a large hierarchy between the masses of spin $\leq 2$ hadrons and spin $\geq 3$ hadrons; (3) suppression of anomalous magnetic moments of hadrons; (4) hadrons with large numbers of very soft partons and no hard partons; (5) suppression of radiated high-transversemomentum gluon jets in high-energy scattering - just to name a few. So this would not work well.

But despite this, it is worth investigating these models, because any insights whatsoever into the difficult problems of QCD would be very valuable. It is in this limited but nonetheless important context that I suggest progress is possible. This kind of work is well underway, and you may want to look at the more recent work that Polchinski and I have been doing [40], as well as the work of Karch and Katz, Kruczenski et al., Belitsky et al., and Erlich, Katz, Son and Stephanov, among others [33, 42, 43, 41, 44].

A second application is to physics of a very different kind. One of the greatest "aw, shucks" moments of my career came with the realization that Randall and Sundrum got here first. If they had not discovered their five-dimensional method of stabilizing the weak-scale hierarchy [45], Klebanov and I would have discovered something analogous in this model. Take this cascading field theory, or one very much like it. Cut it off in the ultraviolet at some scale corresponding to the Planck scale, and couple it to four-dimensional gravity. Then the dual string theoretic description is five-dimensional $A d S$-like supergravity, plus a four-dimensional graviton zero-mode localized near the boundary, with an infrared cutoff at the value of the $A d S$ radius where $k \rightarrow 0$. Unlike the original Randall-Sundrum models, in which the infrared cutoff is imposed by hand on a strictly-AdS metric, here the $A d S$ metric is warped and cuts itself off dynamically at small $r$. This infrared cutoff is nothing more than the confinement and the generation of a mass gap that occurs at an exponentially low scale in this field theory. In particular, if the number of colors at the Planck scale is $k M$, the low-energy confinement scale is the Planck mass times $e^{-\# k}$. Imagine, then, that the theory is coupled somehow to the electroweak model. At high energies it has many colors, but it cascades down until the number of 
colors is small (and it could be either that $g_{s} M \ll 1$, so that supergravity breaks down before the infrared scale, or that $g_{s} M \gg 1$, so that supergravity is valid at all scales.) At the confinement scale, nonperturbative dynamics could easily be imagined to break electroweak symmetry and/or supersymmetry, leading perhaps to a realistic standard model. Essentially, this provides a model for technicolor-like physics (in which the Higgs mechanism is generated dynamically by strong coupling, and the Higgs boson is a composite field.) String-theoretic realizations of these ideas along the lines suggested by H. Verlinde [47] were later considered by Giddings, Kachru and Polchinski [46] (see Kachru's lectures) who embedded the duality cascade into a fully consistent string background. They thereby provided the first complete string-theoretic realization of the Randall-Sundrum approach to the hierarchy, which in these models simply appears as a dual description of dimensional transmutation. (For realistic model-building, one must deal successfully with the massless Goldstone supermultiplet, or with the massive, weakly-interacting gauge boson supermultiplet by which it is eaten.)

Finally, as I alluded to a moment ago, there is another possible application. The standard model itself, being a model with few colors at weak coupling, could easily (with the addition of some massive matter) be at the base of a similar duality cascade. I toyed with such ideas back in 1995, but I found that the generic extension of the standard model with an ultraviolet chain of Seiberg dualities hits a "duality wall": a finite energy scale at which the number of colors diverges [48]. Unfortunately I didn't come across models where this energy scale is infinite, as in the duality cascade of these lectures. Well, I didn't try too hard, honestly. After all, in the days before supergravity duals, it was completely crazy to discuss field theories which had an ever- and rapidly-increasing number of colors in the ultraviolet. What an ugly idea! especially compared to the simplicity of grand unification, with 5 or 10 colors. But now we know differently: this steadily increasing number of colors translates under duality into a perfectly reasonable weakly-curved string-theoretic background. What previously appeared insane now looks surprisingly beautiful. Perhaps it is even true.

\section{References}

\section{References}

1. N. Seiberg, Nucl. Phys. B 435, 129 (1995) [arXiv:hep-th/9411149].

2. M. J. Strassler, [arXiv:hep-th/0309149].

3. K. A. Intriligator and N. Seiberg, Nucl. Phys. B 444, 125 (1995) [arXiv:hepth/9503179]. 
4. R. G. Leigh and M. J. Strassler, Nucl. Phys. B 447, 95 (1995) [arXiv:hepth/9503121].

5. A. Kapustin and M. J. Strassler, JHEP 9904, 021 (1999) [arXiv:hepth/9902033].

6. N. Seiberg and E. Witten, Nucl. Phys. B 431, 484 (1994) [arXiv:hepth/9408099].

7. M. J. Strassler, Prepared for ICTP Spring School on Superstrings and Related Matters, Trieste, Italy, 2-10 Apr 2001

8. P. C. Argyres, K. A. Intriligator, R. G. Leigh and M. J. Strassler, JHEP 0004, 029 (2000) [arXiv:hep-th/9910250].

9. For a review, see the appendix A. E. Nelson and M. J. Strassler, JHEP 0207, 021 (2002) [arXiv:hep-ph/0104051].

10. I. R. Klebanov and E. Witten, Nucl. Phys. B 536, 199 (1998) [arXiv:hepth/9807080].

11. E. Witten, Nucl. Phys. B 500, 3 (1997) [arXiv:hep-th/9703166].

12. P. Candelas and X. C. de la Ossa, Nucl. Phys. B 342, 246 (1990).

13. I. R. Klebanov, Nucl. Phys. B 496, 231 (1997) [arXiv:hep-th/9702076]; S. S. Gubser, I. R. Klebanov and A. A. Tseytlin, Nucl. Phys. B 499, 217 (1997) [arXiv:hep-th/9703040]. S. S. Gubser and I. R. Klebanov, Phys. Lett. B 413, 41 (1997) [arXiv:hep-th/9708005].

14. J. M. Maldacena, Adv. Theor. Math. Phys. 2, 231 (1998) [Int. J. Theor. Phys. 38, 1113 (1999)] [arXiv:hep-th/9711200].

15. For a review of AdS/CFT where this is clearly explained, see O. Aharony, S. S. Gubser, J. M. Maldacena, H. Ooguri and Y. Oz, Phys. Rept. 323, 183 (2000) [arXiv:hep-th/9905111].

16. S. S. Gubser, Phys. Rev. D 59, 025006 (1999) [arXiv:hep-th/9807164].

17. A. Ceresole, G. Dall'Agata, R. D'Auria and S. Ferrara, Class. Quant. Grav. 17, 1017 (2000) [arXiv:hep-th/9910066]; A. Ceresole, G. Dall'Agata and R. D'Auria, JHEP 9911, 009 (1999) [arXiv:hep-th/9907216].

18. S. S. Gubser and I. R. Klebanov, Phys. Rev. D 58, 125025 (1998) [arXiv:hepth/9808075].

19. I. R. Klebanov, P. Ouyang and E. Witten, Phys. Rev. D 65, 105007 (2002) [arXiv:hep-th/0202056].

20. I. Affleck, M. Dine and N. Seiberg, Nucl. Phys. B 241, 493 (1984).

21. I. R. Klebanov and M. J. Strassler, JHEP 0008, 052 (2000) [arXiv:hepth/0007191].

22. N. Seiberg, [arXiv:hep-th/9408013].

23. O. Aharony, JHEP 0103, 012 (2001) [arXiv:hep-th/0101013].

24. S. S. Gubser, C. P. Herzog and I. R. Klebanov, Comptes Rendus Physique 5, 1031 (2004) [arXiv:hep-th/0409186].

25. I. R. Klebanov and A. A. Tseytlin, Nucl. Phys. B 578, 123 (2000) [arXiv:hepth/0002159].

26. I. R. Klebanov and N. A. Nekrasov, Nucl. Phys. B 574, 263 (2000) [arXiv:hep-th/9911096].

27. M. Henningson and K. Skenderis, JHEP 9807, 023 (1998) [arXiv:hepth/9806087]. 
28. M. Krasnitz, JHEP 0212, 048 (2002) [arXiv:hep-th/0209163]; M. Krasnitz, [arXiv:hep-th/0011179].

29. C. P. Herzog, I. R. Klebanov and P. Ouyang, [arXiv:hep-th/0205100].

30. J. Polchinski and M. J. Strassler, [arXiv:hep-th/0003136].

31. E. J. Copeland, R. C. Myers and J. Polchinski, JHEP 0406, 013 (2004) [arXiv:hep-th/0312067].

32. C. Csaki, C. Grojean, H. Murayama, L. Pilo and J. Terning, Phys. Rev. D 69, 055006 (2004) [arXiv:hep-ph/0305237].

33. A. Karch and A. Katz, Fortsch. Phys. 51, 759 (2003).

34. P. Ouyang, Nucl. Phys. B 699, 207 (2004) [arXiv:hep-th/0311084].

35. J. Babington, D. E. Crooks and N. J. Evans, JHEP 0302, 024 (2003) [arXiv:hep-th/0207076].

36. J. Babington, J. Erdmenger, N. J. Evans, Z. Guralnik and I. Kirsch, Phys. Rev. D 69, 066007 (2004) [arXiv:hep-th/0306018].

37. J. L. F. Barbon, C. Hoyos, D. Mateos and R. C. Myers, JHEP 0410, 029 (2004) [arXiv:hep-th/0404260].

38. E. Witten, Adv. Theor. Math. Phys. 2, 505 (1998) [arXiv:hep-th/9803131].

39. C. Csaki, H. Ooguri, Y. Oz and J. Terning, JHEP 9901, 017 (1999) [arXiv:hep-th/9806021].

40. J. Polchinski and M. J. Strassler, Phys. Rev. Lett. 88, 031601 (2002) [arXiv:hep-th/0109174]; JHEP 0305, 012 (2003) [arXiv:hep-th/0209211].

41. D. T. Son and M. A. Stephanov, Phys. Rev. D 69, 065020 (2004) [arXiv:hep$\mathrm{ph} / 0304182]$.

42. M. Kruczenski, D. Mateos, R. C. Myers and D. J. Winters, JHEP 0405, 041 (2004) [arXiv:hep-th/0311270]. M. Kruczenski, L. A. P. Zayas, J. Sonnenschein and D. Vaman, [arXiv:hep-th/0410035]. M. Kruczenski, D. Mateos, R. C. Myers and D. J. Winters, JHEP 0307, 049 (2003) [arXiv:hepth/0304032].

43. A. V. Belitsky, A. S. Gorsky and G. P. Korchemsky, Nucl. Phys. B 667, 3 (2003) [arXiv:hep-th/0304028]; M. Kruczenski, JHEP 0212, 024 (2002) [arXiv:hep-th/0210115].

44. J. Erlich, E. Katz, D. T. Son and M. A. Stephanov, [arXiv:hep-ph/0501128].

45. L. Randall and R. Sundrum, Phys. Rev. Lett. 83, 3370 (1999) [arXiv:hep$\mathrm{ph} / 9905221]$.

46. S. B. Giddings, S. Kachru and J. Polchinski, Phys. Rev. D 66, 106006 (2002) [arXiv:hep-th/0105097].

47. H. Verlinde, Nucl. Phys. B 580, 264 (2000) [arXiv:hep-th/9906182].

48. M. J. Strassler, Prepared for International Workshop on Perspectives of Strong Coupling Gauge Theories (SCGT 96), Nagoya, Japan, 13-16 Nov 1996 